\title{
L'uomo, il suono e la musica
}

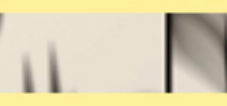

ALESSANDRO BERTIROTTI
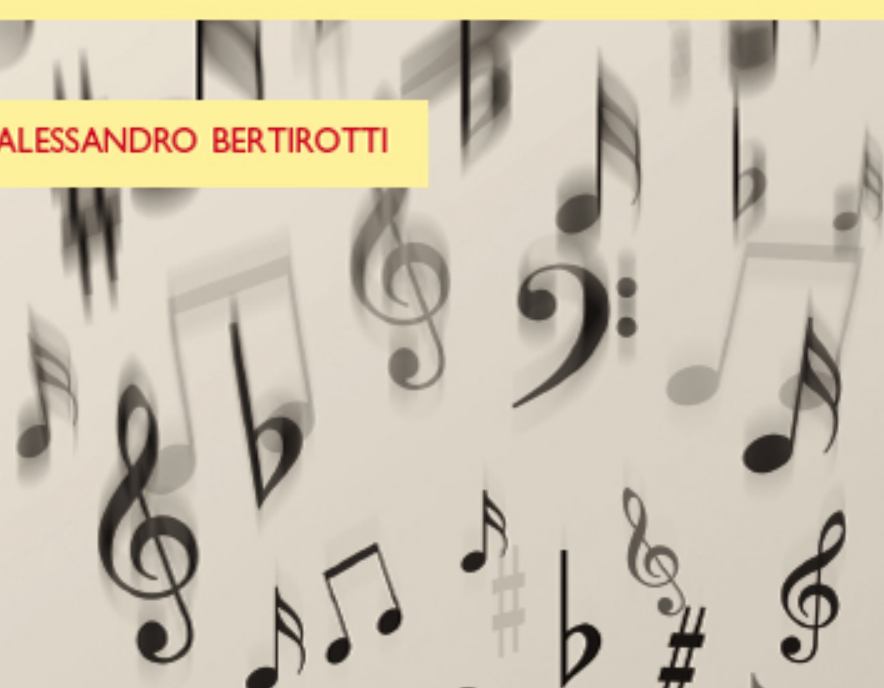
STRUMENTI

PER LA DIDATTICA E LA RICERCA

$-13-$ 

Alessandro Bertirotti

\section{L'uomo, il suono e la musica}

Firenze University Press

2003 
L'uomo, il suono e la musica / Alessandro Bertirotti. Firenze : Firenze University Press, 2003.

(Strumenti per la didattica e la ricerca ; 13)

ISBN 88-8453-064-4 (online)

ISBN 88-8453-073-3 (print)

306.484 (ed. 20)

Antropologia e musica

(C) 2003 Firenze University Press

Università degli Studi di Firenze

Firenze University Press

Borgo Albizi, 28, 50122 Firenze, Italy

http://www.fupress.com/

Printed in Italy 


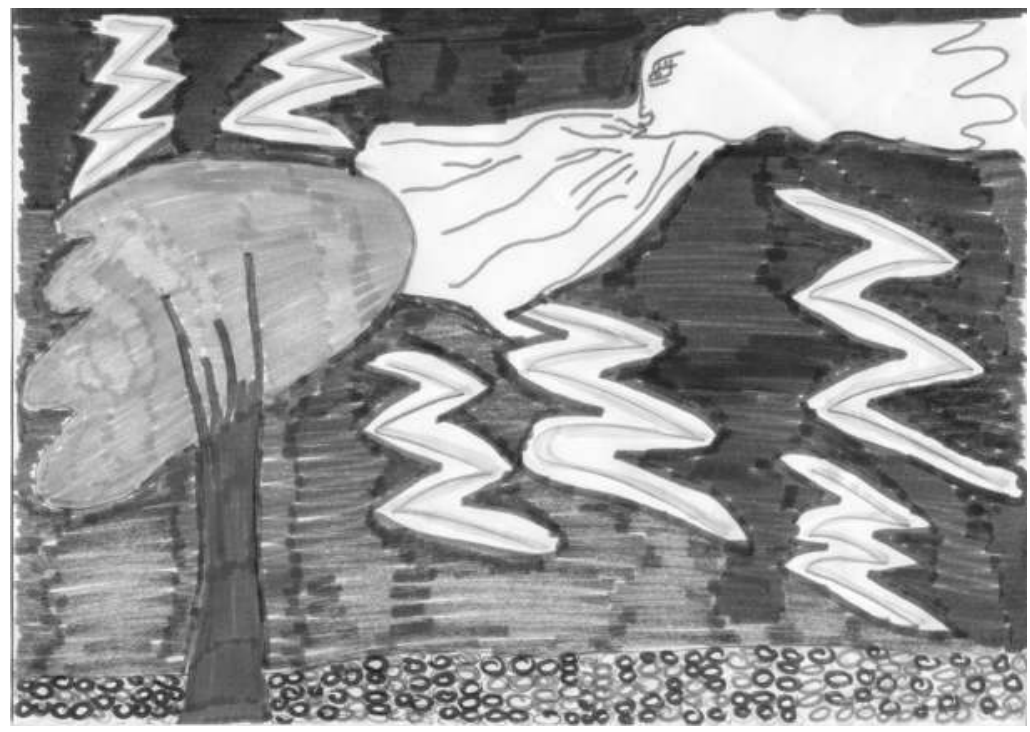

Disegno di Federica Sorrentino, otto anni, 2002, Torino

Sono stati particolarmente interessanti e piacevoli i dibattiti e gli incontri con Gavino Musio, Brunetto Chiarelli, Mario Zunino, Massimiliano Damerini, Luigia Cordati Rosaia, Augusta Larosa, Gabriella Tartarini, Marco Baisi, Isabella Massardo, Roberto Notaris, Silvia Lambiase, Marco Saporiti, Loredana Sausa, Marcello Napoli, Luciano Lanfranchi, Alessandra Zarri, Francesco Risso, Carla Rosaia. Ultimo, ma non per questo meno importante, è Pietro Antonini, la cui vicinanza ha stimolato in me la continuazione di un cammino di ricerca e di studio. 



\section{Sommario}

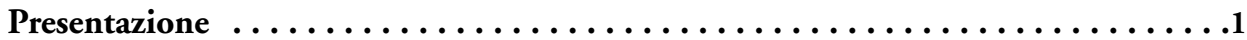

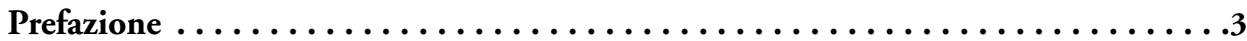

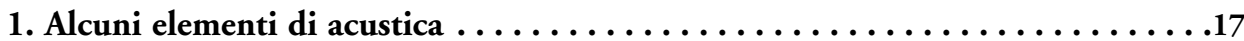

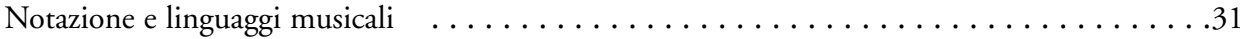

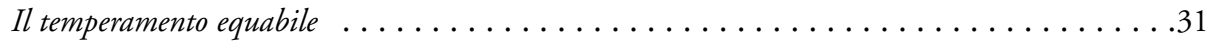

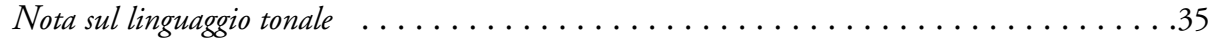

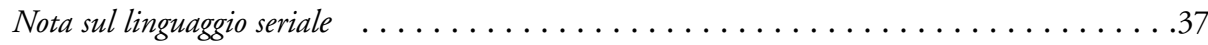

2. Considerazioni sulle origini e l'evoluzione del suono e della musica . . . . . .41

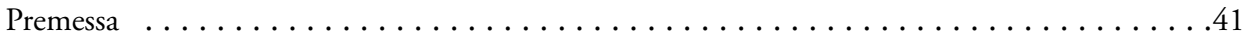

Una relazione circolare suono-uomo-suono $\ldots \ldots \ldots \ldots \ldots \ldots \ldots \ldots \ldots \ldots \ldots \ldots \ldots \ldots \ldots \ldots \ldots \ldots$

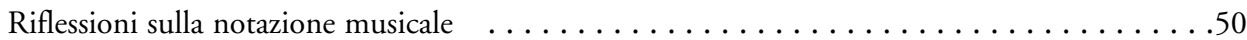



3. Musica e linguaggio: una riflessione sui risultati relativi a questo settore di ricerca recentemente pubblicati dalla New York Academy of Sciences . . . . . . 59

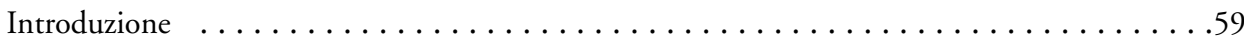

Similitudini e differenze fra il linguaggio e la musica: l'approccio evoluzionistico

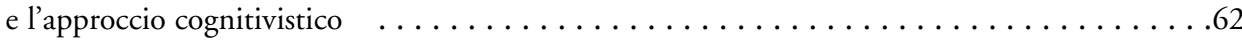

Esiste una vera e propria specificità del linguaggio? . . . . . . . . . . . . . . . . . .69

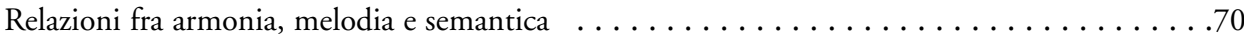

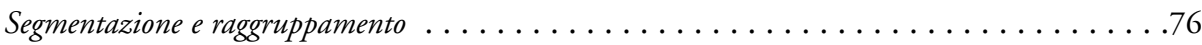

La predisposizione alla regolarità $\ldots \ldots \ldots \ldots \ldots \ldots \ldots \ldots \ldots \ldots \ldots \ldots$

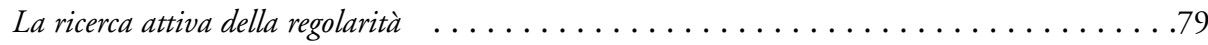

Il livello temporale ottimale di catalogazione degli eventi sonori . . . . . . . . . . . . . .79

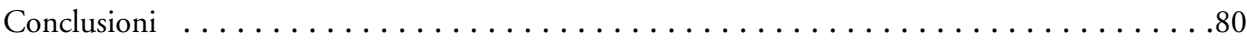

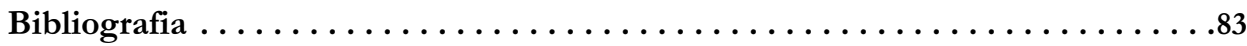





\section{Presentazione}

La musica è una attività che l'uomo organizza intorno ai suoni. L'uomo vive il divenire della propria esistenza in stretto rapporto con l'insieme degli stimoli sonori che lo circonda e questa esperienza funge da elemento integrante del binomio natura e società. Fare e ascoltare musica trasforma variamente il nostro modo di essere nel mondo. L'attività che l'uomo organizza intorno ai suoni lo accompagna dagli albori della civiltà a qualunque cultura esso appartenga.

Il lavoro degli etnomusicologi che ha portato all'ascolto, alla classificazione e allo studio di vari tipi di musica in culture diverse ha consentito la comprensione delle musiche altre, ma anche un avvicinamento delle culture con cui gli studiosi sono venuti in contatto.

Questi studi evidenziano che la musica è praticata da tutte le culture. Questo ci consente ora di valutare il potere che la musica ha nel suscitare emozioni profonde che evidenziano il puro godimento estetico, la gioia e il dolore, la disperazione e l'euforia, la depressione e l'esaltazione, la monotonia della vita quotidiana e la sorpresa di fronte alle novità. In effetti nella musica sono impliciti tutti quei significati emotivi, che selezionati dall'evoluzione, trovano in essa un canale espressivo privilegiato in quanto condiviso culturalmente.

Nel nostro vivere quotidiano spesso sperimentiamo l'impossibilità di esprimere le nostre emozioni quando si presentano in tutta la loro carica emotiva: dobbiamo infatti rispettare precise regole di comunicazione per cui collera, aggressività o anche felicità devono trovare modalità espressive socialmente e culturalmente accettabili. In questa ottica, il fare ed ascoltare musica costituiscono canali di espressione emozionale accettabili che raggiungono, in quanto tali, un alto livello di compartecipazione.

Decodificare un suono comporta anche stabilire condotte per cui la musica diventa un insieme di condotte che comprende il produrla come l'ascoltarla. Vi sono condotte musicali che contengono elementi minacciosi per l'integrità del gruppo mentre altre favoriscono la coesione del gruppo. Il "sistema cultura", pur rivolgendo la propria attenzione ad entrambe, cercherà di isolare le prime e di potenziare le seconde.

La musica si presenta come una fra le tante possibili tecniche comunicative che permette di esprimere se stessi attraverso nuovi "codici linguistici".

Gli elementi della musica vengono elaborati cognitivamente anche grazie all'intervento del linguaggio il quale completa i significati musicali con significati linguistici frutto di ideologie e culture.

Mentre lo sviluppo sia del linguaggio che della musica è il prodotto di una complessa attività neuronale strutturata ed organizzata in quanto prerogativa umana, dagli studi emerge la convinzione che la musica sia associata ad una specifica architettura cerebrale. Le modalità di esecuzione, di comprensione e di fruizione dei suoni fanno parte della fenomenologia culturale, ma il fatto che queste attività appartengano a tutte le culture e che esiste un substrato neurobiologico che le sottende, fanno pensare che siano legate a meccanismi evolutivi quindi a strutture biologiche precise. 
Per questo il confronto tra processi elaborativi che caratterizzano la musica e quelli che caratterizzano il linguaggio può consentire notevoli progressi nella ricerca neurocognitiva.

Una mappatura delle aree cerebrali adibite al linguaggio e alla musica ha evidenziato l'intervento autonomo, indipendente di specifiche popolazioni neuronali. Abilità linguistiche e musicali possono dipendere in parte anche da un training formativo, ma il patrimonio neuronale implicato in queste acquisizioni è lo stesso.

A seguito di queste riflessioni e di questi dati scientifici, l'autore si domanda, innanzi tutto che cosa si intenda per musica, quale posto occupi la musica nella gerarchia dei sistemi comunicativi, quali siano i suoi significanti e i suoi significati e perché sia in grado di soddisfare così intensamente alcuni nostri bisogni intellettuali ed emozionali.

Nel tentativo di fornire queste chiavi di lettura della musica, Alessandro Bertirotti tenta di stimolare una migliore comprensione delle tecniche comunicative musicali, attraverso un'analisi fisica del suono per approdare allo studio delle pratiche socio-musicali, alle condotte sonore tipiche della relazione circolare suono-uomo-suono, motore fondamentale dello sviluppo della specie. Infatti si può pensare che per i primi esseri umani dall'ascolto attento dei suoni e dei rumori, dalla loro localizzazione e dalle conseguenti condotte poteva dipendere la sopravvivenza e si sono quindi evoluti tutti quelli che individuato un suono, un rumore e il pericolo che esso poteva costituire, hanno saputo mettere in atto meccanismi di difesa.

Ne consegue l'esposizione delle attività neuronali e percettive impegnate durante la produzione e l'ascolto dei suoni organizzati ossia della musica.

L'aspetto innovativo di questi contributi è costituito anche dall'individuazione di un percorso in cui la moderna neuroscienza possa impegnarsi nella cognizione musicale al fine di collocare l'assunzione di abilità cognitive musicali in uno schema interpretativo ampio che tenga conto dei diversi livelli di plasticità cerebrale la quale consente di legare le acquisizioni di abilità musicali e del linguaggio a quelle di abilità cognitive generali.

Brunetto Chiarelli

Ordinario di Antropologia

Università di Firenze 


\section{Prefazione}

Il titolo di questo libro, L'uomo, il suono e la musica, suggerisce che esso sembra proporsi, ed il testo lo conferma, quale introduzione ad una ricerca che potrebbe intitolarsi: Introduzione ai fondamenti cognitivi del pensiero musicale e della risposta dei fruitori della musica.

Per quanto la domanda risalga alle origini stesse della produzione e del consumo dell'arte, in essa comprendendo i processi mentali relativi alla musica, questo approccio sembrerebbe ripercorrere il cammino che i filosofi, interrogandosi sul significato dell'arte, hanno racchiuso, già a partire dalla metà del '700, in una disciplina che si sarebbe chiamata estetica ${ }^{1}$. Dal momento che quest'area della filosofia ha coinvolto i pensatori con una suggestione che verosimilmente altre aree non hanno goduto, e che essa ha destato in Italia particolare interesse specie in un filosofo quale Benedetto Croce, il richiamo all'estetica quale "scienza dell'espressione e linguistica generale", potrebbe apparire superfluo quando l'estetica sia intesa come storia dei tentativi di intendere il significato dell'arte ed i processi della fruizione di essa².

Mancando un accordo circa i processi mentali da cui un'opera d'arte scaturisce, e quelli che sovraintendono al suo consumo estetico, o "godimento dell'opera", il discorso potrebbe dirsi qui praticamente chiuso. Ciò sarebbe giustificato dal solo titolo del testo di Bertirotti, ma non dal secondo quale si è proposto in principio e che scaturisce dal suo contenuto: una cosa è parlare dal punto di vista mentale dell'arte quale produzione e/o consumo, invocando canoni estetici che non hanno mai risolto veramente il problema, ed altra è parlare di processi mentali indotti dal rapporto con l'arte, muovendo dalla premessa che quel rapporto e quei processi appartengono ad una scienza della mente che si chiama cognitiva. La capacità euristica di essa si fonda su un decorso storico e su un apparato metodologico che gli storici dell'estetica non potevano conoscere, ma che presenta il pregio di portare il problema nello specifico ambito della mente e dei suoi concreti percorsi, sottraendolo ad una sempre ricorrente tentazione metafisica.

Quella che è stata chiama la "nuova scienza della mente", non soltanto costituisce una nuova barriera contro le tentazioni astratte, ma ha confermato che queste persistono anche nelle scienze che più di altre dovrebbero, in un quadro globale, rifondare la conoscenza dell'uomo. Ci si riferisce a discipline oggi significativamente in crisi quali la psichiatria, la psicologia, la sociologia, la criminologia, pur se ancora una pletora di scuole specialistiche continua a inventare terapie per patologie che sovente si autoalimentano e sembrano ignorare la sempre più viva presenza delle neuroscienze e della scienza della cultura.

\footnotetext{
${ }^{1}$ Ci si riferisce al filosofo tedesco Alexander G. Baumgarten (1714-1762). Cfr. Croce B., 1902, Estetica, Laterza, Bari, pagg. 233-241.

2 Il Croce è ritornato a lungo sul problema dell'Estetica. Si veda, oltre il testo citato, Croce B., 1912, Breviario di Estetica, Laterza, Bari, e, in forma sintetica, Estetica in nuce, confluito poi nel Breviario di Estetica.

${ }^{3}$ Gardner H., 1985, The Mind's New Science, Basic Books Inc., New York, trad. it 1988, La nuova scienza della mente. Storia della rivoluzione cognitiva, Feltrinelli, Milano.
} 
Questa premessa si giustifica con lo sviluppo delle scienze della mente e specie sulle istanze che hanno indotto l'autore di questo volume a scriverlo.

A metà degli anni '80, in un colloquio con chi scrive, docente di antropologia culturale all'Università di Firenze, l'autore aveva espresso il desiderio di unire al diploma in pianoforte, una laurea in antropologia culturale con una tesi che approfondisse il senso e la natura della carica emotiva che in lui si manifestava dopo diverse ore che studiava il suo autore preferito, F. Chopin, e che cresceva sino ad uno stato di effettiva prostrazione.

Se la richiesta nasceva dalla intensità delle sue reazioni, egli si rendeva già conto che la prostrazione era l'aspetto macroscopico di un fenomeno più complesso, che egli allora riferiva soltanto a se stesso, ma che, essendo tutt'altro che occasionale, rispondeva a qualcosa che aveva luogo nella mente e di cui egli non riusciva a farsi un'idea.

Il fenomeno non era nuovo, ed era stato osservato, più che rispetto all'esperienza della musica, a quella dell'arte figurativa e della poesia. È noto come Dostojevski, che sembra fosse epilettico, venisse colto da un attacco dopo una lunga esposizione ad un quadro che lo coinvolgeva profondamente. È del pari conosciuto il bisogno di certi lettori di imparare a memoria le poesie preferite e di ripeterle ossessivamente, come avrebbe detto Maupassant, "partout, ainsi qu'une prière".

Qualche anno fa, in certi visitatori di musei di Firenze, città essa stessa concentrato di opere d'arte, si era osservato che dopo uno o due giorni di visite venivano colti da amnesia, senso di angoscia e prostrazione. La sindrome fu definita dalla psicologa che più particolarmente se ne occupò (Graziella Magherini) "sindrome di Stendhal", in quanto lo scrittore francese aveva descritto per primo i sintomi confusionali che aveva provato dopo una esposizione troppo intensa e specie concentrata alle opere d'arte.

Riguardo alla letteratura, chi scrive aveva potuto rilevare che segni della sindrome potevano comparire in lettori di poesia e specie di testi letterari come il racconto o, comunque, brevi. In altri lettori lo stesso componimento non suscitava nulla, ma l'intensa emozione e le manifestazioni connesse non si verificavano se l'asseto linguistico del racconto veniva alterato intervenendo sul linguaggio dilatandone, sia pure limitatamente, la struttura formale. Il che poteva significare che il linguaggio, ad esempio di F. Chopin, poteva essere quello e soltanto quello, e che la mobilitazione del sistema nervoso del ricettore poteva avvenire soltanto se il messaggio metteva in sufficiente contatto due strutture: quella mentale o neuromentale dell'emittente e quella del ricettore.

Tutto ciò era ovvio, o lo era in apparenza. Ma, ci si chiedeva, quali strutture e funzioni gli stavano dietro? Dal punto di vista cognitivo si potevano seguire i canoni della scienza della mente. Essi erano complessi e fortemente articolari, alcuni aspetti fondazionali potevano essere proposti a Bertirotti per impostare il suo problema.

Il saggio di Bertirotti può considerarsi un trattato essenziale di scienza della musica o musicologia o anche, per gli ampi riferimenti al rapporto uomo-suono e dunque alla relazione musica-sistema nervoso umano, di bio o neuromusicologia. Il saggio è diviso in tre capitoli, 1 Elementi di acustica, 2 Sulle origini e l'evoluqione del suono e della musica, 3 Similitudine e differenze tra il linguaggio e la musica. Completa il testo una ricca documentazione linguistica (cap. 3) che conduce ad una discussione sulla distinzione tra approccio evoluzionistico ed approccio cognitivistico al linguaggio musicale. 
Il volume è pertanto decisamente tecnico e per molti rispetti destinato ad un pubblico di specialisti e di musicologi. In realtà, come si è notato, esso presenta una fisionomia più ricca della semplice istanza tecnico-musicale e che può apparire a due facce, nel senso che se quella tecnica è la più immediatamente leggibile, essa è pervasa da un interrogativo nel quale la discussione sulle procedure e sul linguaggio musicale implica, da un lato, l'esistenza di un costruttore dell'opera musicale, da un altro, di un fruitore di essa. Entrambi potranno chiedersi, come si era già posto a suo tempo l'autore: "Ma, in definitiva, che cosa accade dentro di noi quando creiamo o godiamo un'opera musicale?".

Questo interrogativo già travalica l'ambito propriamente musicologico per investire il problema della rappresentazione linguistico-simbolica e della sua fruizione da parte di un recettore in qualunque ambito espressivo e cioè, in senso generale, artistico.

Il lettore non riceve risposta nel volume, come non l'aveva avuta comprensibilmente molti anni prima lo stesso autore, quando aveva proposto un lavoro di tesi sulla violenta emozione e sui relativi processi, "dentro di me", che si mobilitavano dopo aver eseguito per diverse ore appunto F. Chopin.

Allora chi scrive aveva ritenuto che la sorta di orgasmo mentale che coglieva il giovane musicista quando eseguiva i "Notturni", in quanto reazione parossistica alla lunga esposizione ad un'opera musicale e dunque al suo autore, fosse difficilmente traducibile in una tesi di laurea. A ciò aggiungasi che, se trattavasi di una delle possibili forme della universale fruizione dell'arte, il fenomeno poteva apparire grezzamente psicologico, mentre era, in realtà, cognitivo.

Quando Bertirotti propose la sua tesi nel marzo del 1986, la scienza cognitiva era ancora nella fase psicologica. Era cioè largamente impregnata di interesse per il rapporto mente-informatizzazione, ma non era ancora avvenuta la svolta che, attraverso un ampio rapporto interdisciplinare, avrebbe veduto due aree fondazionali divenire protagoniste di The Mind's New Science ${ }^{4}$, vale a dire la neurobiologia e la scienza della cultura.

Anche se a chi scrive era noto che le neuroscienze si erano già mobilitate in senso che sarebbe stato denominato cognitivistico, e che, ad esempio, il premio Nobel per la Fisiologia, Edgar Douglas Adrian aveva pubblicato nel 1952 Phisical Background of Perception, e che nel 1953 Charles Sherrington, anch'egli premio Nobel per la fisiologia, e lo stesso Adrian avevano curato una raccolta di studi con contributi tra gli altri di A.J. Ayer e di G. Ryle, dal significativo titolo The Physical Basis of Mind $^{5}$, quel che ancora negli anni '70-'80 mancava era il coordinamento formale, oltre che fenomenologico, tra le neuroscienze e la scienza della cultura.

Questo spiega perché l'interesse di chi scrive si fosse orientato verso una direzione affine a quella che aveva esordito nello stesso periodo negli Stati Uniti e che era scaturita da

\footnotetext{
${ }^{4}$ Cfr. Gardner H., op. cit., cap. 3, specie pagg. 55-59.

${ }^{5}$ Adrian E.D., 1952, The Physical Backgound of Perception, University Press, London, trad. it. 1953, I fondamenti fisiologici della percezione, Einaudi, Torino; Adrian E.D. e Sherrington C., 1953, The Physical Basis of Mind, Basic Blokwell, Oxford, trad. it. 1953, Le basi fisiche del pensiero, Einaudi, Torino. Cfr. Ayer A.J., 1963, The Foundations of Empirical Knowledge, Macmillan, Ventura; Ryle G., 1960, Dilemnas, Cambridge University Press, London.
} 
Gavino Musio

un grosso impegno di ricerca ma dal versante opposto, vale a dire da quello delle scienze sociali e dunque anche dall'antropologia culturale e dalla psicologia sociale.

Si trattava della teorizzazione che il sociologo Talcott Parsons aveva avviato già al principio degli anni '50 attraverso una rivisitazione dello storicismo tedesco contemporaneo ed in particolare del Max Weber. Parsons si proponeva una teoria generale dell'azione sociale che avrebbe dovuto avere al centro la fenomenologia della società (sociologia) e, collateralmente, quella della psiche (psicologia) e quella della cultura (antropologia culturale). Nonostante un impegno durato oltre venti anni, sino alla morte nel 1966, Parsons credette nella sua teoria dell'azione di base sociologica ed anche se, sin da allora, avrebbe avuto come teoria scarso riconoscimento ${ }^{6}$.

Tra il 1954 e il 1958, Parsons, con l'antropologo Clyde Kluckhohn, diresse, in un'area del Texas meridionale, un'ampia ricerca socio-antropologica. Da essa sarebbe scaturita una serie di volumi, ma specialmente uno, a cura di una antropologa, Florence Kluckhohn e collaboratori, nel quale essa poneva le basi, in collaborazione teorica con il marito Clyde e attraverso una serie di matrici oggi classiche ${ }^{7}$, di una teoria generale ed interdisciplinare della cultura ${ }^{8}$. Quella teoria sarebbe stata inserita in un'opera collettanea e fondamentale che sarebbe apparsa, nel 1958, con contributi di biologi e neurobiologi, antropologi, etc., con significativo titolo Verso una teoria unificata del comportamento umano?.

\footnotetext{
${ }^{6}$ Parsons T., Shils E.A. and others, 1951, Toward a General Theory of Action, Harper \& Row, New York. Ci si chiederà perché ricordare Parsons, sia pure in modo essenziale. Perché se oggi ci riteniamo più prossimi a rispondere alla domanda di Bertirotti, ciò è da attribuirsi al fatto che sin dall'esordio ottocentesco della sociologia, della psichiatria, della psicologia, dell'antropologia culturale, della criminologia, della psicoanalisi e più tardi delle neuroscienze e della epistemologia, lo sviluppo di queste scienze è stato segnato da un impegno via via più profondo verso una teoria della mente in una dimensione sempre più interdisciplinare. Mentre gli approcci verso una "nuova scienza della mente" sino agli anni 1950 si svolsero in genere su base unidisciplinare, Parsons, tra teorizzazione di gruppo, dibattiti, ricerche sul terreno, mobilitò direttamente almeno trenta tra i più autorevoli studiosi di scienze umane degli anni '50 degli Stati Uniti, ma indirettamente stimolò convegni, ricerche e pubblicazioni che per il rigore segnarono un'epoca e un metodo. Negli '60 e '70 l'impegno verso la scienza della mente sarebbe passato agli psicologi, e dopo gli anni ' 80 alle neuroscienze, unitamente appunto alla scienza della cultura. La cultura resta pertanto la fenomenologia di base e cioè un contenuto primario della struttura neurologica dell'uomo e la logica della sua struttura, in quanto schema dell'organizzazione dei contenuti del cervello-mente, costituisce un modo, vale a dire fonda la specificità delle risposte individuali e di gruppo agli input che gli provengono dall'interno o dall'esterno, sia pure con il contributo di una molteplicità di fattori e processi la cui descrizione confluisce nella scienza della mente. In questa fase si assiste, del resto già da alcuni decenni, ad un flusso di pubblicazioni sulle quali il giudizio può essere ancora incerto. Un criterio discriminante sempre valido sembra essere quello per cui tra neuroscienze e scienze della cultura non dovrebbe esserci più spazio per astrazioni. Cfr. anche Gallino L., 1978, voce Azione sociale, in Dizionario di Sociologia, UTET, Torino.

${ }^{7}$ Kluckhohn C., 1949, Mirror for man, McGraw-Hill, New York; Kluckhohn C., 1951, Values and Value Orientations in the Theory of Action, in Parsons T. \& Shilds E.A., (Eds.), 1951, Toward a General Theory of Action, Harvard University Press, Cambridge, Mass., pagg. 388-433; Kluckhohn C., 1954, Culture and Behavior, in Lindzey G., (Ed.), 1954, The Handbook of Social Psychology, vol. 2. Addison-Wesley, Reading, Mass.; Kluckhohn C., 1956, Toward a Comparison of Value-Emphases in Different Cultures, in White L.D., (Ed.), 1956, The state of the social sciences, University of Chicago Press, Chicago: pagg. 116-32; Kluckhohn C. \& Murray H.A., 1948, Personality in Nature, Culture and Society, Knopf, New York; Kluckhohn F., Strodtbeck F., Roberts J.M., Romney K., 1960, Variations in Value Orientations. A Theory Tested in Five Cultures, Cambridge.

${ }^{8}$ Kluckhohn F., Strodtbeck F., Roberts J.M., Romney K., 1960, Variations in Value Orientations. A Theory Tested in Five Cultures, Cambridge.

${ }^{9}$ Grinker G., (a cura di) 1958, Toward a Unified Theory of Human Behaviour, New York.
} 
Nonostante, a muovere almeno dalla metà dell'Ottocento, il rapporto tra la scienza e la modernità, sia pure con notevoli differenze a seconda delle aree di conoscenza, abbia proceduto anche ad un rigetto della ostinata tendenza a passare dal concreto all'astratto e dunque dal fisico al metafisico, non tanto nelle scienze propriamente fisiche ma in quelle denominate umane, un terreno nel quale questa tendenza sembra tenacemente persistere è quello del rapporto tra il pensiero e la mente e dunque tra la mente e il cervello.

Per quanto succintamente, in questa sede converrà ribadire che la proposizione che il cognitivista americano Marvin Minsky affacciava quasi contemporaneamente alla sintesi del Gardner: "La mente è ciò che il cervello fa" ${ }^{10}$, significa anche che il nostro essere nel mondo e la nostra relazione con esso è resa possibile da un apparato di infinitesimali elementi che hanno la capacità di reagire al mondo, di restarne impressionati come lastre fotografiche, di ricordarlo e di riprodurlo nei tanti modi possibili come immagini nello schermo mentale e con le innumerevoli integrazioni e correlazioni. Senza questo apparato non potremmo conoscere e dire nulla del mondo, ma neppure di noi stessi.

Il cervello, e nello specifico la corteccia, è il risultato di un lungo tirocinio formativo evolutivo specie per la parte destinata a reagire al mondo, per cui esso è sempre in movimento e accrescimento ${ }^{11}$.

Spesso le esperienze del cervello sono mere ripetizioni tautologiche, e che pertanto mobilitano sempre le stesse cellule, tutto quel che pensiamo può essere pensato nella misura in cui disponiamo di sistemi di cellule specializzate e organizzate relativamente al tema che dobbiamo trattare e cioè pensare.

Nell'arco di due milioni e mezzo di anni ${ }^{12}$, ogni individuo ed ogni generazione hanno fatto una quantità progressiva di esperienze. Ogni esperienza ne ha prodotte altre. Alle nuove esperienze di generazioni corrisponderebbe la formazione di nuovi neuroni specializzati su quel tipo di ontologia o tema-esperienza: ogni individuo ha fatto le sue esperienze utilizzando i neuroni di cui disponeva e che egli ha arricchito rendendoli disponibili per un certo incremento di esperienze. L'incremento avverrà senza che il numero dei suoi neuroni aumenti.

Questa più ampia capacità acquisita di pensiero-esperienza del suo cervello si tradurrebbe nella sua discendenza biologica, a cominciare dai discendenti diretti, in un cervello che sarà, almeno per la metà, simile al suo, ma dotato di un maggior numero di neuroni deputati a quelle o queste aree cognitive che il padre ha esperimentato ed arricchito. Sembra essere questo, in termini elementari, il meccanismo ontofilogenetico con cui si è sviluppata la corteccia umana dai circa 400 grammi dei primi ominidi, ai circa 1400 dell'Homo sapiens sapiens attuale ${ }^{13}$.

\footnotetext{
${ }^{10}$ Minsky M., 1985, The Society of Mind, Mass. Institute of Technology, trad. it. 1989, La società della mente, Adelphi, Milano.

${ }^{11}$ Tobias Ph., 1991, Evolution of the Human Brain in Man: the Tottering Biped, University of Adelaide Press, South Australian, trad. it. 1992, "L'evoluzione del cervello, dell'intelleto e dello spirito dell'uomo", in Il Bipede barcollante, Einaudi, Torino.

${ }^{12}$ Tobias Ph., ibidem.

${ }^{13}$ Tobias Ph., ibidem.
} 
Questo processo è verosimilmente uno dei più complessi dell'intero apparto cervellomente, nel senso che le rappresentazioni del mondo della vita siano trasferite selettivamente, nell'apparato neuronale ed in questo esse vivano arricchendosi di continuo, con il contributo di una serie di fattori dei quali ci sono noti quelli che consideriamo fondamentali.

Essi possono essere individuati e descritti, ma converrà rilevare che la concorrenza dei diversi fattori si traduce in una complessità di funzioni e operazioni che non sempre vengono presi in considerazione collegialmente dagli studiosi delle diverse aree disciplinari.

Non è evidentemente questa la sede per affrontare una ricostruqione cognitiva dal momento che si esita ancora a fondarla, come si è notato, sulla rivisitazione del lessico relativo. Vi è ancora notevole incertezza sui termini che denotano aree di conoscenza metafisiche come anima e persino spirito ed aree di conoscenza empirica come cultura. Bertirotti presenta della cultura la prospettiva più cognitiva che risale alle ricordate ricerche del gruppo Parsons-Kluckhohn del 1955 che rivendicavano la disposizione mentale innata della cultura, arricchita per scelte nella vita secondo un gruppo di categorie (spazio, tempo, azione, relazione, mondo, etc.). Ciò significa che gli atteggiamenti mentali verso la cultura sono appunto in ogni società patrimonio innato e dunque ontofilogenetico di scelte. Con la cultura, la nostra cultura, pertanto noi nasciamo e nel corso della vita evolviamo con essa.

Qualche biologo risale ancora alla vecchia concezione della cultura come struttura soltanto appresa. Il che significherebbe che, con un po' di buona volontà, chiunque potrebbe "diventare ad esempio ebreo", e magari di un'altra epoca, mentre chiunque abbia frequentato un ebreo "di quattro quarti" non ha tardato ad accorgersi che quell'ebreo, o quell'inglese, o quel fiorentino, è nato ebreo, inglese o fiorentino, e che rappresentano una stratificazione di epoche mentali-culturali, molte delle quali essi stessi possono non essersi neppure accorti di possedere.

Il meccanismo ontofilogenetico che si è descritto è reso funzionale, più che dai fattori che contribuiscono a farlo vivere, dalla sua estrema plasticità. E questa viene a sua volta consentita dalla sinergica partecipazione di tutti quei fattori: nel senso che ad ogni atto del cervello-mente partecipano, con incidenze di volta in volta diverse, tutti i fattori appunto.

Un atto del cervello-mente consiste appunto nella risposta a qualunque tipo di imput, quale che ne sia il modo con cui viene inviato. E dunque anche al messaggio estetico o che noi chiamiamo artistico, a cominciare dal contatto con la musica e dalla sua fruizione ${ }^{14}$.

Il concetto di atto può essere utilizzato con vantaggio quando si intenda mettere ordine nella nostra osservazione di un universo (universo II), quale quello mentale che, dopo quello fisico (universo I), è stato considerato come il fenomeno più complesso che possa cadere sotto gli occhi dell'uomo.

Un atto è una quantità di azione compiuta dal cervello-mente. Esso è dunque un pattern e contiene, secondo il suo oggetto, tutti gli elementi o fattori che il rapporto cervellomente-oggetto comporta e che intervengono secondo i fini che quel rapporto si propone.

\footnotetext{
${ }^{14}$ Cfr. Musio G., 1995, La mente culturale. Struttura della cultura e logica della organizzazione della mente, Università di Firenze, pagg. 122-137.
} 
Nel caso del rapporto di un individuo con un messaggio ad esempio artistico-musicale, il primo fine del sistema cervello-mente è quello di produrre una data reazione. E questo atto può essere necessariamente aperto o chiuso, completo o incompleto, inserito in un contesto di breve, media o lunga durata, e specialmente difeso o no contro i suoi maggiori nemici, vale a dire gli input che, pur in apparenza scorrendo nello stesso binario, sono violenza degradata e cioè dei virus. Pochi artisti hanno potuto difendere la costruzione della propria armonia mentale sino alla fine, per intenderci, dall'anti-Chopin. Ogni pseudo musica, o pseudo letteratura, o pseudo scienza, corrode l'armonia della mente e dell'identità, dato che essere "eletti" significa anche difendersi nella tempesta provocata da coloro che sono stati soltanto "chiamati".

Il fatto che in un secondo momento il fruitore si interroghi sul processo mobilitato dal suo interesse, apre un nuovo atto che si congiunge al primo, che potrà essere a sua volta completo o incompleto e che potrà aprire, ciò che più conta, una catena di atti legati fra loro $\mathrm{da}$ una affinità-proporzione matematica che li costituiranno come sistemi o pattern mentali dotati di struttura. Le affinità-proporzioni sono date dalle strutture e dalla interna dinamica delle variabili di ogni atto.

È inutile scendere qui, per i limiti di spazio, al quadro matematico delle strutture degli atti. Importa soltanto richiamare alcuni dei fattori sui quali si fonda ogni pattern, o atto, o nucleo di esperienza (Erlebnis) ${ }^{15}$ dotato di tutti i componenti necessari che possano intervenire in una situazione nella quale la ricezione di un messaggio sia tanto più rilevante per un fruitore.

In un modello cognitivo generale, costituito da un insieme di fattori (o variabili), si situano due fenomenologie o strutture dominanti: la fenomenologia neurobiologica e la fenomenologia culturale ${ }^{16}$. Esse si sviluppano nella lunga durata della vita dell'individuo e procedono insieme. Questo procedere, per la conoscenza che ne abbiamo, appare soggetto ad un controllo adattante reciproco.

In altre parole, la specificità e la capacità dell'apparato neuronale di rispondere ad un dato input (nel nostro caso una rappresentazione musicale o comunque artistica) ci informerebbe in primo luogo che quell'apparato neuronale è già costruito per reagire al messaggio secondo le incidenze categoriali della cultura in cui si è costruito il suo sistemacapacità di reagire all'input. Il messaggio non arriva in modo inerziale, ma deve essere adatto e insieme adattato al sistema neuroculturale che lo riceve. In pratica, se F. Chopin giunge ad un sistema neurocorticale e induce profonde reazioni, ciò significa che il modulo musicale Chopin, od il suo reticolo di base (divenuto la sua essenza), senza chiamarsi tale, era già presente in quel sistema neuro-culturale. E questo, in definitiva, lo attendeva, in

\footnotetext{
${ }^{15}$ Dilthey W., 1947, Esperienza vissuta e poesia, Istituto Editoriale Italiano, Milano.

${ }^{16} \mathrm{Si}$ tenga presente che l'apparato neurobiologico è una fenomenologia al tempo stesso empirica e cognitiva. Essa è cioè empirica perché composta dall'apparato neuronale, e cognitiva perché come si è accennato, è in grado di rappresentare la realtà, elaborarla e riprodurla. È in grado cioè di essere mente. Come tale il patrimonio di rappresentazione della realtà (il patrimonio cognitivo della mente) è organizzato secondo una logica che è la logica del sistema culturale. E questa è categoriale e dunque organizzativa della vita comunitaria in cui la mente di un individuo è immersa, e pertanto dei suoi stessi contenuti in quanto rappresentazioni della realtà. In termini elementari, questo è il quadro funzionale del modello che si è denominato brain-mind-culture-system.
} 
quanto nelle sue coordinate dominanti, era già dentro il fruitore, e ciò che più conta in quel momento o sino ad allora, le alternative di altri reticoli musicali potevano essere in lui poche o non esistere ancora (ma questo è un altro capitolo del problema).

L'esperienza del ricettore, cioè, e dunque la continua transazione tra gli input dall'esterno ed il suo apparato neuro-culturale, era già orientata verso quel tipo di input, era cioè selettiva e tale era divenuta in un rapporto circolare o transattivo pregresso, per cui la scelta di F. Chopin, come fonte di input di particolare intensità, era già stata fatta da una serie di atti di esperienza di dimensione ed intensità progressivamente maggiore, via via che la disponibilità neuroculturale a Chopin si perfezionava ${ }^{17}$.

La scelta di Chopin era in definitiva Chopin stesso che si attivava nel suo esecutore. Risalendo al percorso di questa esperienza e ricostruendo il reticolo degli atti in successione, non dovrebbe essere difficile tracciare un primo pattern della sua identità mentale.

Nel succinto quadro della odierna scienza cognitiva che si è proposto nella Prefazione a suggerito dal dialogo con Alessandro Bertirotti e dal suo colloquio con la musica, si è accennato al contributo della matematica alla fondazione della scienza della mente.

La matematica come tale ed i contributi alla conoscenza scientifica fondati sul cosiddetto empirismo logico e specie sull'analisi del linguaggio applicati allo studio della mente, appartengono ad un ambito di conoscenza che suole essere denominato di filosofia della mente $^{18}$. Va subito chiarito che l'approccio matematico alla conoscenza della mente e dei suoi processi è anche qui puramente strumentale, vale a dire tecnico. Ciò significa che l'apparato paradigmatico che qui si segue si fonda sul principio della teorizzazione e della relativa verifica". "Le teorie sono strumenti o reti che si gettano per conoscere" ${ }^{19}$. Se la rete resta vuota, se ne dovranno costruire delle altre.

Il lungo travaglio di pensiero che nel XX secolo ha avuto inizio dopo la ottocentesca critica alla geometria euclidea ${ }^{21}$ specie dopo la critica al concetto tradizionale di scienza da

\footnotetext{
${ }^{17}$ Questo complesso sistema di rapporti tra due strutture mentali operanti reciprocamente su notevoli disposizioni di affinità, non sembra accadere nell'apparato bioumano, a cominciare dal cervello, in modo inerziale. I messaggi che giungono alla corteccia sembrerebbero filtrari-selezionati secondo affinità e grado di disponibilità della corteccia per essi, ma l'apparato che sovrintende alla selezione sembrerebbe risiedere in un'altra area del cervello, detta area limbica. Quest'area, avvertono i neurobiologi (Changeux J.P., 1983, L'homme neuronale, Libraire Arthème, Paris, trad. it. 1986, L'uomo neuronale, Feltrinelli, Milano, pag. 134; Boncinelli E., 1999, Il cervello, la mente e l'anima, Mondadori, Milano, pagg. 214-218), tra le molte funzioni ha quella di caricare i messaggi di tensione (emotività), dunque preparandoli per la corteccia, vale a dire per quel che essa può recepire. Questo approccio confermerebbe una delle ipotesi più suggestive, a sostegno della quale si dispone peraltro di discrete conferme: l'area limbica seleziona e adatta $i$ messaggi per l'area corrispondente della corteccia, e quest'ultima confermerebbe e consoliderebbe transattivamente l'adattabilità dell'area limbica stessa a selezionare i messaggi adatti ad essa.

${ }^{18}$ Cfr. Nannini S., 2002, L'anima e il corpo. Una introduzione storica alla filosofia della mente, Laterza, Bari-Roma, specie il cap. 4. Per una prospettiva di insieme con la relativa integrazione storica ed interdisciplinare cfr. D’Agostini F., 1999, Breve storia della filosofia del Novecento. L'anomalia paradigmatica, Einaudi, Torino, specie i cap. VII, La filosofia scientifica e IX, La filosofia analitica.

${ }^{19}$ D'Agostini F., op. cit., pag. 148.

${ }^{20}$ Popper K., 1934, Logic of Scientific Explanation, trad. it. 1970, La logica della scoperta scientifica, Einaudi, Torino, pag. 43.

${ }^{21}$ Hilbert D., 1970, Fondamenti della Geometria, Feltrinelli, Milano.
} 
parte del francese Jules Poincarée ${ }^{2}$, fu in effetti, nella molteplicità delle correnti, anche un tentativo di fondare scientificamente la conoscenza sottraendola alla tentazione metafisica. Di qui la rilevanza di Popper come filosofo della scienza e la fondazione epistemologica della scienza cognitiva che ne consegue.

È significativo che Popper abbia ripetutamente affrontato il problema della mente, della sua funzione e l'approccio cartesiano al apporto mente-corpo ${ }^{23}$. Ma mentre non risulta che egli si sia mai occupato della cultura come fenomenologia antropo-mentale, è rilevante che uno dei maggiori critici del metodo popperiano, K. Feyerabend (1970), rifiutando alfine la sua stessa critica radicale al Popper, abbia anche indicato nella cultura (antropologica) la fenomenologia sulla quale si sarebbero dovute basare le ricerche sui fondamenti stessi della conoscenza ${ }^{24}$.

Direttamente o indirettamente, altri si erano accostati alla fenomenologia culturale come fattore condizionante e caratterizzante la conoscenza scientifica, a cominciare dal Th. G. Kuhn ${ }^{25}$, dal W. Quine ${ }^{26}$ e, specie, dal secondo Wittgenstein delle Ricerche filosofiche ${ }^{27}$.

Per quanto con ricorrenti emersioni e scomparse nella attenzione degli scienziati, a muovere dalla metà del Novecento, la fenomenologia della cultura è apparsa in modo sempre più insistito praticamente in tutte le ontologie che riguardino in qualche modo l'entità uomo.

Se Wittgenstein avesse avuto natura di antropologo, dopo aver rifiutato la proposta del Tractatus del 1923, secondo la quale il linguaggio costituisce la rappresentazione logica del mondo, ed avere scoperto che il linguaggio significa in ogni società secondo il costume e le usanze (il significato di una parola è l'uso nel linguaggio) e dunque secondo la cultura, avrebbe dovuto contribuire anche allo sviluppo di una scienza la cui disarmante complessità fenomenologica veniva comunemente nascosta dalla apparente persuasività della sua superficie $^{28}$.

\footnotetext{
${ }^{22}$ Poincaré J. H., 1962, La Science et l'Hypothèse, Flammarion, Paris, (1902).

${ }^{23}$ Cfr. di Popper K., 1992, Tre saggi sulla mente umana 1978-1987, Vallecchi Editore, Firenze. Anche il secondo volume (del 1977) del dialogo Eccles J. C.-Popper K., 1986, L'io ed il suo cervello. Struttura e funzioni cerebrali, Armando, Roma. Ancora (del 1994) di Popper K., 1996, La conoscenza ed il rapporto corpo-mente, Il Mulino, Bologna, specie i capp. I, V e VI. Il richiamo a Popper è programmatico. Come in ogni scienza che preveda un congruo periodo di vitalità produttiva, anche sulla scienza cognitiva si è riversata una quantità di pubblicazioni non di rado mancanti, prima che del merito, della prima e sintomatica carenza che è la interdisciplinarità. Forse mai, nel decorso della conoscenza moderna, ci si è trovati di fronte ad una esperienza interdisciplinare così diversificata. Si pensi soltanto al primo rapporto quale è quello neurobiologia-cultura, con le molte implicazioni. Nel 1985, H. Gardner ha affrontato il problema, pur essendo consapevole dei rischi ma anche della mancanza di alternative. Nel suo già citato The Mind's Nen Science, su 439 pagine più la bibliografia di oltre 600 titoli, si contano 17 discipline distribuite nel testo con incidenze decrescenti. Nelle scienze cognitive la collaborazione tra diversi autori appare forse più ardua che il tentativo di procedere da soli con un dato metodo: ma quale? Per questo il riferimento a Popper offre in primo luogo una difesa contro le fughe dal rigore ed un continuo controllo appunto contro le tentazioni metafisiche, anche inconsapevoli.

${ }^{24}$ Cfr. Feyerabend P. K., 28 Aprile 1996, Addio al relativismo. Ogni cultura è in potenza tutte le culture, Il Sole 24 Ore, pag. 29.

${ }^{25}$ Kuhn T., 1978, La struttura delle rivoluzioni scientifiche, Einaudi, Torino.

${ }^{26}$ Quine W., 1960, Manuale di logica, Feltrinelli, Milano.

${ }^{27}$ Wittgenstein L., 1999, Ricerche Filosofiche, Einaudi, Torino.

${ }^{28}$ Soltanto con la scienza della mente si sarebbe giunti a comprendere che la fenomenologia culturale costituiva il fondamento normativo e logico dell'identità mentale dell'individuo. L'apparente ovvietà della fenomenologia culturale
} 
Il concetto di elemento minimo o atomo del reale fisico risale alle origini stesso del pensiero occidentale, nel tentativo di opporre, al fluire inerziale degli eventi, frazioni della realtà che costituissero insieme una quantità osservabile ed una sosta nell'inarrestabile corsa del divenire. Nel mondo fisico, vale a dire nell'Universo I, i risultati hanno confermato i primi assunti. Da Democrito a Leibniz, da Einstein ad Heisenberg a Plank: l'universo fisico non soltanto è apparso composto di particelle di dimensioni sempre minori, e interamente complesse, ma la sua struttura è apparsa relativa; l'energia che lo caratterizza e dunque la sua realtà risulta intrinsecamente discontinua; qualunque osservazione su di essa trova nella sua interna struttura fisica i limiti della stessa capacità di conoscere.

Come sappiamo, la tragicità dei risultati non ha bloccato lo sviluppo della fisica teorica, né tra i più sensibili è aumentato il numero dei suicidi.

Diverso è stato nelle scienze dette umane il tentativo di soddisfare la stessa esigenza, vale a dire di riuscire a pensare l'Universo II, la mente dell'uomo cioè ed i suoi percorsi, secondo una entità pensante, un pattern, vale a dire il cervello ${ }^{29}$.

Dal punto di vista dei processi mentali e cognitivi, ci si rese conto già negli anni 198590 che la difesa della scienza cognitiva e dei suoi molti nemici, stava certo nella sua fondazione paradigmatica, ma questa, come in ogni scienza, doveva rappresentare in modo corretto la sua fenomenologia. Ciò poteva cominciare, o essere favorito, dalla quantificazione delle ricerche su di essa e dunque anche dei risultati.

Trattatasi cioè di fondare la rivoluzione cognitiva su una base, da un lato, di rigore della ricerca e, dall'altro, dell'impianto epistemologico.

Questo è stato, a muovere dalla fine degli anni '80, l'impegno primario nella ricerca nei diversi settori teorici e applicativi della disciplina condotti dalla sezione cognitiva dell'AISA (Associazione Interdisciplinare di Scienze Antropologiche, Istituto di Antropologia dell’Università degli Studi di Firenze, via del Proconsolo, 12).

Muovendo dalla rivisitazione del primo cognitivista ufficialmente riconosciuto, quale lo storicista W. Dilthey con i suoi approfondimenti della conoscenza della mente e specie con i suoi concetti di Erlebnis e di Verstehen ${ }^{30}$, il gruppo di studiosi si mosse all'elaborazione dei paradigmi ed allo studio dei settori di applicazione della scienza della mente in una pro-

ha non di rado in alcuni sociologi e pedagogisti una bizzarra azione di rigetto, evidentemente ignorando i Parsons, $\mathrm{i}$ Sorokin, i Thomas, i Lynd, etc. Ma è potuto anche accadere che qualche docente di Antropologia Culturale sia sfuggito al concetto di identità, sostanzialmente dichiarando che esso non esiste e che una casa editrice di prestigio gli abbia pubblicato un testo dal titolo eloquente: Remotti, F., 1996, Contro l'identità, Laterza, Bari-Roma. Il che è come dire che le menti di tutti gli uomini sono identiche come le carpe clonate, e che gli ebrei, le SS, i Toscani, i Nambikwara sono tutti la stessa cosa.

${ }^{29}$ Walter W.G., 1957, The Living Brain, G. Duckwosth Editions, London, trad. it. 1957, Il cervello vivente, Feltrinelli, Milano. Per il concetto di pattern ed il cervello come pattern (o sistema spazio-temporale) cfr. cap. III, pagg. 55-70. Risalire alla letteratura neurobiologica nel quadro del brain-mind-culture-system appare qui non funzionale, in gran parte per il fatto che questa produzione, per iniziativa degli stessi neurobiologi, si divide in testi altamente specializzati, come ad esempio i contributi in questa materia del MIT (Massachuset Institute of Technology) e quelli linguisticamente adattati ad un previsto impiego interdisciplinare. Si veda per questo il ricordato Changeux J.P. (1994), per il MIT Damasio A., (1994) e, tra i più fecondi in Italia, Oliverio A. (1995). Converrà inoltre ricordare come summa delle neuroscienze Kandal E.R., Schwartz J.H., Jessel T.M., 1991, Principles of Neural Science, (3a edizione), Elsevier Science Publication, trad. it. 1994, Principi di neuroscienze, Edizioni Ambrosiana, Milano, pagg. 1113.

${ }^{30}$ Dilthey W., 1947, Esperienza vissuta e poesia, Istituto Editoriale Italiano, Milano. 
spettiva della quale la ricerca di Alessandro Bertirotti costituiva uno dei significativi percorsi.

Non essendovi qui spazio per uno approfondimento, converrà elencare soltanto le diverse aree seguite a Firenze, oltre quella di Bertirotti: la neurobiologia cognitiva e la culturologica, come il citato brain-mind-culture-system; le applicazioni dei moduli cognitivi di ricerca ai processi mentali ed alle patologie mentali culturali indotte (non psicotiche) e, specie, allo studio delle identità mentali individuali e di gruppo; di qui una serie di applicazioni delle identità mentali a situazioni patologiche di origine sessuale giovanile (II reparto di Urologia dell'Azienda Ospedaliera di Careggi, Firenze), la patologia e la prevenzione del dolore umano (Centro di Terapia Antalgica dell'Ospedale Civile di Prato, "Prevenzione e difesa dal dolore", corso propedeutico diretto dalla dott.ssa Maria Ciampolini).

Inoltre una serie di applicazioni: storiografia cognitiva (collaborazione con la Regione Toscana, On. Riccardo Nencini, Unione Europea e fattore umano. Dalla cultura delle nazioni alla cultura delle regioni); la Kultur germanica e lo sterminio; politologia cognitiva; cognitivistica delle situazioni estreme; della grande industria; la fondazione cognitiva dei colori e la loro utilizzazione nell'industria tessile (M. Chemeri).

Resoconti dei risultati, della teorizzazione e dei metodi impiegati, delle tecniche di rilevazione, dei modelli e dei campioni, continuano a comparire in pubblicazioni specifiche.

La scienza cognitiva nasce da una concordanza interdisciplinare, come si è osservato: ma le neuroscienze da sole non sono sufficienti. Cosi non lo è la scienza della cultura. Così non lo sono tutti $i$ campi che confluiscono in essa. Si tratta di disporre di un metodo, ma anche questo, se è tanto, in questa materia sembra non bastare. Occorre pervenire anche ad un pattern, vale a dire a un qualcosa che potrebbe definirsi immaginazione cognitiva, come in ogni scienza, nel senso in cui un brillante sociologo americano, Wright Mills, aveva coniato nel 1959 l'espressione epistemologica Sociological imagination. Cfr. Mills W., 1959, "The Sociological Imagination", Oxford University Press, New York, trad. it. 1962, "L’immaginazione sociologica”, Il Saggiatore, Milano.

Gavino Musio

Ordinario di Antropologia culturale

Università di Firenze 


\section{Bibliografia}

Adrian E.D., 1952, The Physical Backogound of Perception, University Press, London, trad. it. 1953, I fondamenti fisiologici della percezione, Einaudi, Torino.

Adrian E.D. e Sherrington C., 1953, The Physical Basis of Mind, Basic Blokwell, Oxford, trad. it. 1953, Le basi fisiche del pensiero, Einaudi, Torino.

Ayer A.J., 1963, The Foundations of Emperical Knowledge, Macmillan, Ventura.

Boncinelli E., 1999, Il cervello, la mente e l'anima, Mondadori, Milano.

Changeux J.P., 1983, L'homme neuronale, Libraire Arthème, Paris, trad. it. 1986, L'uomo neuronale, Feltrinelli, Milano.

Croce B., 1902, Estetica, Laterza, Bari.

Croce B., 1912, Breviario di Estetica, Laterza, Bari.

D’Agostini F., 1999, Breve storia della filosofia del Novecento. L'anomalia paradigmatica, Einaudi, Torino.

Del Carlo I., 2001, Il concetto di Kultur nel pensiero storicistico tedesco, tesi di laurea, Univ. di Firenze, Fac. di Scienze della Formazione, relatore Musio G.

Dilthey W., 1947, Esperienza vissuta e poesia, Istituto Editoriale Italiano, Milano.

Eccles J.C., Popper K., 1986, L’io ed il suo cervello. Struttura e funzioni cerebrali, Armando, Roma.

Feyerabend P.K., 28 Aprile 1996, Addio al relativismo. Ogni cultura è in potenza tutte le culture, Il Sole 24 Ore.

Gallino L., 1978, voce Azione sociale, in Dizionario di Sociologia, UTET, Torino.

Gardner H., 1985, The Mind's New Science, Basic Books Inc., New York, trad. it 1988, La nuova scienza della mente. Storia della rivoluzione cognitiva, Feltrinelli, Milano.

Grinker G., (a cura di), 1958, Toward a Unified Theory of Human Behaviour, New York.

Lelli G., (a cura di), 1955, Aristotele, Organon, Einaudi, Torino, vol. I.

Hilbert D., 1970, Fondamenti della Geometria, Feltrinelli, Milano.

Mills W., 1959, The Sociological Imagination, Oxford University Press, New York, trad. it. 1962, L'immaginazione sociologica, Il Saggiatore, Milano.

Minsky M., 1985, The Society of Mind, Mass. Institute of Technology, trad. it. 1989, La società della mente, Adelphi, Milano.

Musio G., 1995, La mente culturale. Struttura della cultura e logica della organizzazione della mente, Università di Firenze.

Nannini S., 2002, L'anima e il corpo. Una introduzione storica alla filosofia della mente, Laterza, Bari-Roma.

Parsons T., \& Shils E.A. and others, 1951, Toward a General Theory of Action, Harper \& Row, New York.

Poincaré J.H., 1962, La Science et l'Hypothèse, Flammarion, Paris, (1902).

Popper K., 1934, Logic of Scientific Explanation, trad. it. 1970, La logica della scoperta scientifica, Einaudi, Torino.

Popper K., 1992, Tre saggi sulla mente umana 1978-1987, Vallecchi Editore, Firenze.

Popper K., 1996, La conoscenza ed il rapporto corpo-mente, Il Mulino, Bologna.

Quine W., 1960, Manuale di logica, Feltrinelli, Milano. 
Remotti F., 1996, Contro l'identità, Laterza, Bari-Roma.

Ryle G., 1960, Dilemnas, Cambridge University Press, London.

Tobias Ph., 1991, Evolution of the Human Brain in Man: the Tottering Biped, University of Adelaide Press, South Australian, trad. it. 1992, L'evoluzione del cervello, dellintelletto e dello spirito dell'uomo, in Il Bipede barcollante, Einaudi, Torino.

Kandal E.R., Schwartz J.H. e Jessel T.M., 1991, Principles of Neural Science, (3a edizione), Elsevier Science Publication, trad. it. 1994, Principi di neuroscienze, Edizioni Ambrosiana, Milano.

Kluckhohn C., 1949, Mirror for man, McGraw-Hill, New York.

Kluckhohn C., 1951, Values and Value Orientations in the Theory of Action, in Parsons T. \& Shilds E.A., (Eds.), 1951, Toward a General Theory of Action, Harvard University Press, Cambridge, Mass., pp. 388-433.

Kluckhohn C., 1954, Culture and Behavior, in Lindzey G., (Ed.), 1954, The Handbook of Social Psychology, vol. 2. Addison-Wesley, Reading, Mass.

Kluckhohn C., 1956, Toward a Comparison of Value-Emphases in Different Cultures, in White L.D., (Ed.), 1956, The state of the social sciences, University of Chicago Press, Chicago: pp. 116-132.

Kluckhohn C. \& Murray H.A., 1948, Personality in Nature, Culture and Society, Knopf, New York.

Kluckhohn F., Strodtbeck F., Roberts J.M., Romney K., 1960, Variations in Value Orientations. A Theory Tested in Five Cultures, Cambridge.

Kuhn T., 1978, La struttura delle rivoluzioni scientifiche, Einaudi, Torino.

Walter W.G., 1957, The Living Brain, G. Duckwosth Editions, London, trad. it. 1957, Il cervello vivente, Feltrinelli, Milano.

Wittgenstein L., 1999, Ricerche Filosofiche, Einaudi, Torino. 



\section{Alcuni elementi di acustica}

"I segreti della natura si rivelano più pronta-
mente quando vengono tormentati dall'arte
che quando sono lasciati a se stessi."
Ruggero Bacone (1214?-1294)

I rapporti che esistono tra la fisica e la musica ci interessano qui solo in riferimento agli eventi fisici che determinano la percezione del suono ${ }^{1}$.

Riteniamo utile ricordare in questa sede alcune semplici e fondamentali nozioni di acustica, cioè di quel ramo della fisica che studia la produzione e la propagazione del suono: sembra infatti che difficilmente possa esser fatta una riflessione sulla musica prescindendo da tali elementari conoscenze.

1 - Se si ricerca l'origine di un suono qualsiasi, si trova che esso è sempre dovuto alle vibrazioni di una sorgente sonora: può essere generato dalla vibrazione di corde (corde vocali dell'uomo, di un pianoforte o di un violino, ecc.) o di colonne d'aria (organo, clarino, ecc.) o di piastre e membrane (tamburi, altoparlanti, etc.).

L'aria che si trova nelle immediate vicinanze di uno qualsiasi di questi corpi vibranti viene alternativamente compressa e rarefatta e queste perturbazioni vengono trasmesse dall'aria stessa sotto forma di onde elastiche (onde acustiche o sonore) che si allontanano dalla sorgente che le ha prodotte, ma torneremo su questo argomento al punto 2; per il momento ci limitiamo alla constatazione che, se l'onda acustica incontra l'orecchio umano, penetra nel suo interno e determina la sensazione uditiva.

Osserviamo subito che non sempre un corpo che oscilla emette un suono: un corpo pesante che oscilla sospeso ad un filo non produce un suono. Perché il nostro orecchio percepisca un suono occorre che la vibrazione che lo produce sia abbastanza rapida ma non troppo: più precisamente, bisogna che la frequenza delle vibrazioni sia compresa allincirca tra 16 e 12.000 Hert:

FREQUENZA: è il numero delle oscillazioni compiute dal corpo vibrante in un secondo.

PERIODO: è il numero che esprime (in secondi) il tempo impiegato dal corpo vibrante per compiere un'oscillazione completa.

Se con T si indica il periodo di una vibrazione e con $\mathrm{N}$ si indica la sua frequenza, allora

$$
N=\frac{1}{T}
$$

HERTZ: è l'unità di frequenza (un'oscillazione completa al secondo)

\footnotetext{
${ }^{1} \mathrm{Nel}$ vocabolario di elettroacustica è riportata la seguente definizione di suono: "Sensazione uditiva determinata da vibrazioni acustiche" avente carattere soggettivo, e quella di "Vibrazioni acustiche capaci di determinare una sensazione uditiva" avente carattere oggettivo. In Musicoterapia, per suono si intende: a) moti vibratori che determinano delle sensazioni uditive; b) sensazioni uditive determinate dalla ricezione di onde di pressione, cioè i suoni, derivanti dalle vibrazioni di corpi sonori. Cfr. Righini P., 1970, L'acustica per il musicista. Fondamenti fisici della musica, Edizioni Zanibon, Padova, pag. 5.
} 
Fu Galileo a scoprire che la sensazione del suono si produce in noi quando arrivano al nostro orecchio, attraverso l'aria, onde elastiche di frequenza compresa entro certi limiti.

Le onde elastiche di frequenza inferiore a 16 Hertz, o maggiore di 12.000 Hertz, pur giungendo al nostro orecchio, non sono in grado di provocare alcuna sensazione uditiva. Per l'individuo medio, il suono di circa 20 Hertz è molto basso e un suono vicino ai 10.000 Hertz viene percepito come un fischio acutissimo. Può accadere tuttavia che persone molto giovani possano percepire suoni di frequenza anche molto più elevata (fino a 20.000 Hertz).

Si chiamano ultrasuoni le onde elastiche che hanno una frequenza più alta di quella percepibile dall'orecchio umano.

Con semplici esperienze è possibile verificare che in genere noi sentiamo un suono tanto più acuto quanto maggiore è la sua frequenza.

L'aria è uno dei mezzi attraverso i quali il suono può propagarsi. La classica esperienza del campanello elettrico posto sotto la campana di una macchina pneumatica ${ }^{2}$ serve a dimostrare che, in assenza di un mezzo elastico, il suono non si trasmette.

Ma qualunque altro corpo, sia esso solido, liquido o gassoso, è capace di trasmettere le vibrazioni sonore: per esempio, è parte della nostra esperienza quotidiana il fatto che i rumori passano attraverso le pareti, i muri e le finestre chiuse delle nostre case ed è facile constatare che, se in mare si immerge la testa sott'acqua, si può sentire il rumore di un'elica di un'imbarcazione in moto nelle vicinanze.

Quando un suono passa da un mezzo all'altro, esso penetra solo in parte nel secondo mezzo ed in parte viene invece riflesso.

Tra acqua e aria (o inversamente) la parte trasmessa è una piccolissima frazione della quantità riflessa: i suoni subacquei non escono dall'acqua e i suoni aerei non penetrano in essa. È come se il mondo subacqueo e il mondo aereo fossero acusticamente isolati uno dall'altro. E tuttavia, i suoni si propagano nell'acqua meravigliosamente, meglio che nell'aria e più velocemente (la loro velocità è circa 4,4 volte maggiore).

2 - Immaginiamo di avere una laminetta flessibile di acciaio, fissata ad uno dei suoi estremi con l'altro estremo libero: indichiamo con $\mathrm{E}$ il punto in cui si trova l'estremo libero della laminetta quando questa è in riposo (in equilibrio).

Se, applicando la necessaria pressione, spostiamo l'estremo libero della laminetta dalla posizione $\mathrm{E}$ alla posizione $\mathrm{A}$ (vedi la Fig. 1) e poi lo lasciamo, questo estremo si muoverà da $A$ verso $B$, poi nuovamente da $\mathrm{B}$ verso $\mathrm{A}$, compiendo così un'oscillazione completa intorno al suo punto di equilibrio; poi si muoverà nuovamente $\mathrm{da} A$ verso $\mathrm{B}$, poi tornerà in A e così via: oscillerà cioè intorno al punto di equilibrio $\mathrm{E}$.

\footnotetext{
${ }^{2}$ Se si estrae rapidamente l'aria dalla campana mentre il campanello sta suonando, si sente il suono affievolirsi sino a diventare quasi impercettibile; se si fa rientrare l'aria, si sente nuovamente il suono con l'intensità di prima. Il suono non si spenge del tutto per due buone ragioni: la prima è che non si può estrarre tutta l'aria dalla campana (non si può ottenere il vuoto assoluto), la seconda è che il suono viene trasmesso dal campanello alla sua base d'appoggio e da questa alla macchina pneumatica che lo trasmette all'esterno.
} 
Se la frequenza della vibrazione della laminetta è compresa nell'intervallo indicato al punto 1, il nostro orecchio percepisce un suono: l'onda elastica è, in questo caso, un'onda acustica. Ma come è stato trasmesso questo suono al nostro orecchio? Vediamo.

Quando l'estremo libero della laminetta si muove da E verso A, esso spinge lo strato d'aria che gli sta immediatamente vicino, comprimendolo; questi comprime a sua volta lo strato d'aria che lo circonda, il quale ne comprime un terzo e così di seguito: intanto successivamente ciascuno strato ritorna alla condizione normale. Si ha dunque una compressione che si propaga nell'aria, allontanandosi in tutte le direzioni.

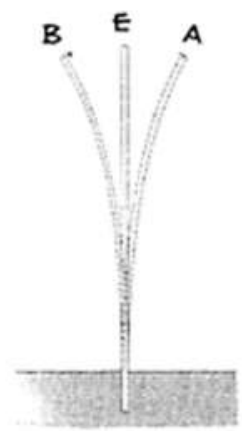

Fig. 1. Oscillazione completa che compie la laminetta (da A verso $B$ e da B verso A), passando dal punto di equilibrio $\mathrm{E}$

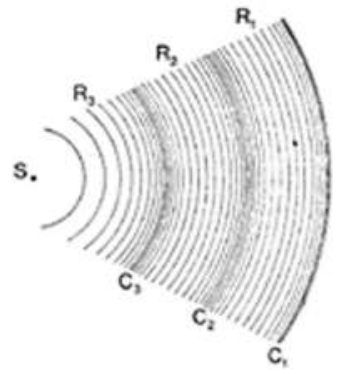

Fig. 2. I momenti di compressione e rarefazione prodotti dalle onde di pressione provenienti dalla fonte sonora $S . R_{3}, R_{2}$ e $R_{1}$ sono i momenti di rarefazione, mentre $C_{3}, C_{2}$ e $C_{1}$ sono di compressione

Negli istanti successivi, quando l'estremo libero della laminetta si muove da A verso E poi da E verso B, lo strato di aria che lo circonda, e che prima era stato compresso, viene ora decompresso e si rarefà; lo strato successivo si precipita a colmare questa rarefazione e ne lascia una al suo posto; questa viene compensata dal terzo strato e così di seguito. Abbiamo ora una rarefazione che si propaga nell'aria a seguito della compressione. Quando poi l'estremo della laminetta ritorna in A (questo avviene dopo un periodo), si forma un nuovo strato di compressione, seguito da un nuovo strato di rarefazione, e così via di seguito.

Si può concludere che la vibrazione della laminetta genera un'onda elastica periodica, costituita da una successione di strati nei quali l'aria è alternativamente compressa e rarefatta (Fig. 2) e che si dilatano in modo che ogni rarefazione insegua la precedente compressione.

Lo stesso avverrebbe ponendo un diapason in vibrazione davanti all'imboccatura di un tubo aperto ad entrambi gli estremi (vedi la Fig. 3); una successione di compressioni e rarefazioni si propagherebbe nel tubo, con la differenza che, mentre nell'aria libera queste si attenuano rapidamente, dentro il tubo procedono anche per grandi cammini senza variare in modo sensibile.

Si ha un fenomeno simile a quello sopra descritto se si fa cadere un sassolino sulla superficie tranquilla dell'acqua di uno stagno (o dell'acqua contenuta in un recipiente): si 


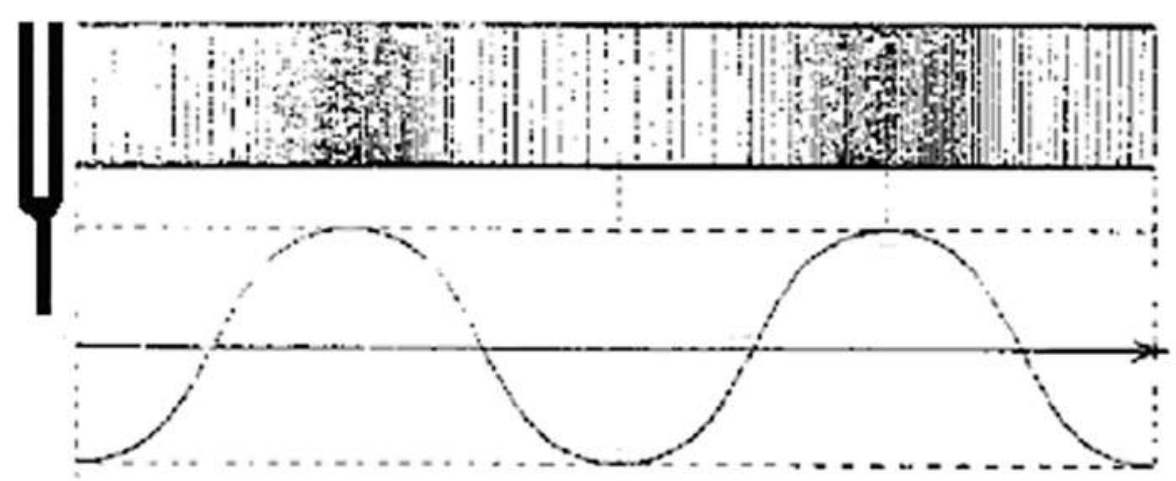

Fig. 3. La successione di compressioni e rarefazioni lungo un tubo messo di fronte alla sorgente diapason. Sotto il tubo si ha la notazione sinusoidale dell'onda sonora, frutto di rarefazioni e compressioni.

osserverà in questo caso una successione di onde che si rincorrono sulla superficie dell'acqua in tutte le direzioni senza raggiungersi mai, attenuandosi man mano sino a svanire. In questo caso, invece di compressioni e rarefazioni dell'aria, si hanno innalzamenti ed abbassamenti della superficie dell'acqua. È proprio da questa analogia che deriva il nome di onda attribuito all'insieme di una compressione e di una rarefazione in un mezzo elastico.

LUNGHEZZA D'ONDA:
è la distanza che si ha tra due successivi strati compressi o tra due
successivi strati rarefatti; si tratta
evidentemente della distanza percorsa dalla perturbazione in un
periodo, cioè nel tempo che impiega una particella, per esempio
di aria, per compiere un'oscillazione completa.

Riflettiamo adesso sulle onde acustiche emesse da una sorgente sonora S, che per comodità immaginiamo puntiforme (vedi la Fig. 4). Consideriamo il moto di una particella d'aria posta ad una certa distanza dalla sorgente sonora (per es. nel punto E), sotto l'azione delle onde acustiche provenienti da S.

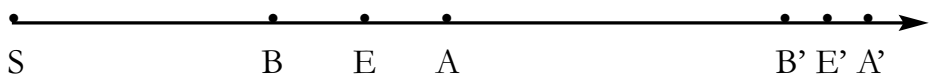

Fig. 4. Moto della particella d'aria E

Questa particella oscilla tra due posizioni A e B compiendo una oscillazione completa (cioè andando dalla posizione di riposo $\mathrm{E}$ ad $\mathrm{A}$, e poi da $\mathrm{A}$ a $\mathrm{B}$ e ritornando in $\mathrm{E}$ ) nel tempo in cui lo strato di compressione e quello di rarefazione passano per il punto E. La particella d'aria vibra quindi nella direzione SE (che è appunto la direzione di propagazione dell'onda acustica emessa da S e passante per E): il suo movimento oscillatorio avviene nella stessa direzione di propagazione dell'onda sonora. 
Si esprime questo fenomeno dicendo che le onde sonore sono onde elastiche longitudinali.

Tutte le particelle di aria (o di qualunque altro corpo) vibrano, dunque, sotto l'azione delle onde sonore. È facile però constatare che queste vibrazioni diventano sempre meno ampie quanto più ci si allontani dalla sorgente sonora (l'energia meccanica emessa dalla sorgente viene infatti distribuendosi su superfici sempre maggiori quanto più l'onda si allontana dalla sorgente stessa). Così una particella d'aria che si trovi nel punto E' (Fig. 4), a maggiore distanza da $\mathrm{S}$ rispetto alla particella situata in $\mathrm{E}$, vibrerà tra due posizioni $\mathrm{A}^{\prime} \mathrm{e}$ B' che sono più vicine tra loro di quanto non siano le posizioni A e B tra le quali oscilla la particella situata nel punto E: l'ampiezza delle vibrazioni va dunque diminuendo man mano che ci si allontana dalla sorgente sonora.

AMPIEZZA di una vibrazione: è il massimo spostamento di una particella vibrante dalla sua posizione di equilibrio.

Osserviamo ancora che le onde elastiche emesse da una sorgente sonora e le onde provocate dalla caduta di un sassolino su una superficie liquida in quiete manifestano tra loro una importante differenza e una importante analogia.

- LA DIFFERENZA: le onde sonore sono longitudinali (come abbiamo visto sopra), mentre le onde che si propagano sulla superficie dell'acqua nel caso sopra descritto sono onde trasversali.

Si può verificare facilmente l'ultima affermazione mettendo un pezzetto di sughero sulla superficie liquida percorsa dalle onde: al passaggio dell'onda il sughero subirà uno spostamento dalla posizione di equilibrio $E$ verso l'alto (innalzamento) fino a raggiungere un'altezza massima A (Fig. 5); successivamente si abbasserà, tornando alla posizione di equilibrio $\mathrm{E}$ per poi proseguire l'abbassamento sino a raggiungere un'altezza minima B; poi si innalzerà di nuovo sino a tornare in E e così via. Gli spostamenti del sughero avvengono in direzione verticale, perpendicolarmente alla direzione (SE) di propagazione dell'onda proveniente da $S$ e passante per $E$, e rappresentano visibilmente lo stesso movimento al quale viene sottoposta al passaggio dell'onda una particella d'acqua situata nel punto E: questo si esprime appunto dicendo che si tratta di un'“onda trasversale”.

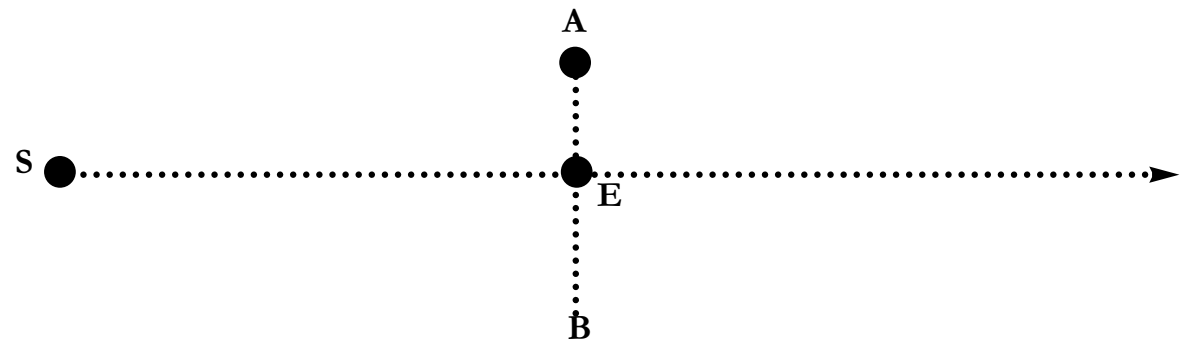

Fig. 5. Moto trasversale della particella E, in direzione di A e di B. Questi spostamenti, ortogonali rispetto alla direzione SE, rappresentano ad esempio il movimento di una particella d'acqua. 
Osserviamo che sono onde trasversali anche quelle che si propagano lungo una corda tesa che venga scossa da un estremo (o pizzicata).

In generale:

- si ha un'onda trasversale quando il movimento trasmesso dall'onda alle particelle del mezzo è perpendicolare alla direzione di propagazione dell'onda;

- si ha un'onda longitudinale quando il movimento trasmesso dall'onda alle particelle del mezzo ha la stessa direzione del raggio di propagazione dell'onda.

- L'ANALOGIA: nelle onde acustiche, così come nelle onde liquide sopra descritte, non c'è trasporto di materia.

Quando una particella del mezzo nel quale l'onda si propaga viene raggiunta dall'onda, essa viene stimolata dall'energia che la raggiunge e compie un movimento elastico periodico (longitudinale nel primo caso, trasversale nel secondo): lungo il percorso di queste onde c'è solo trasmissione di energia. La stessa esperienza del sughero descritta sopra mette in evidenza questa proprietà nel caso dell'onda che si propaga su una superficie liquida: il sughero infatti si solleva e si abbassa, ma non si allontana dalla sorgente.

3 - I caratteri che distinguono un suono dall'altro sono L'ALTEZZA, L'INTENSITÀ, LA DURATA E IL TIMBRO.

- L'ALTEZZA è il carattere del suono che distingue i suoni acuti dai suoni gravi. È dunque quell'attributo della sensazione uditiva per mezzo del quale i suoni possono essere ordinati dal basso verso l'alto, per esempio come avviene nella scala musicale.

Dal punto di vista propriamente fisico, si suole dire che l'altezza di un suono dipende dalla sua frequenza. Più precisamente: quanto maggiore è la frequenz̧a di un suono, tanto più il suono è acuto.

I suoni molto gravi hanno una frequenza di alcune decine di Hertz, mentre i suoni più acuti hanno frequenze di alcune migliaia di Hertz. Nelle Figure 6 e 7 sono rappresentati sul piano cartesiano due suoni puri (vedi al punto 5): la loro rappresentazione grafica, dove si sono posti in ascissa $\mathrm{i}$ tempi e in ordinata la variazione di pressione, è una curva che viene chiamata sinusoide.
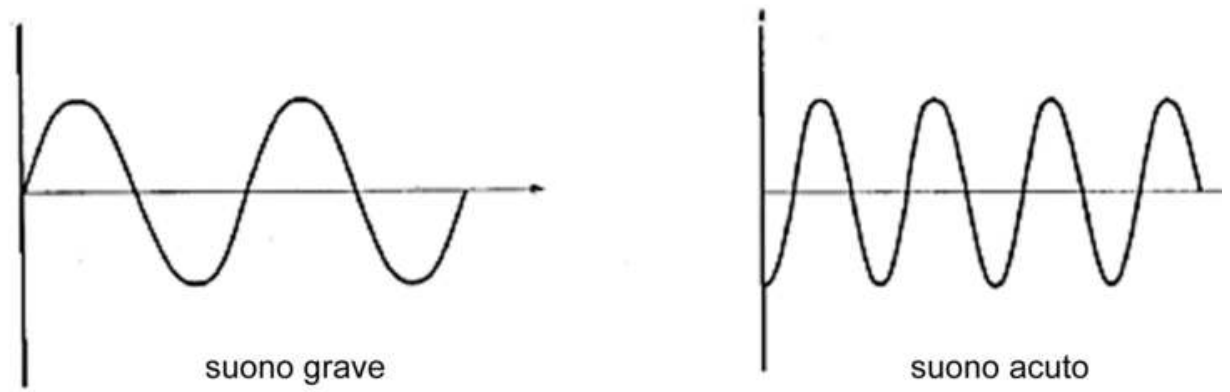

Fig. 6. L'altezza di un suono dipende dall'ampiezza della frequenza: tanto maggiore è la frequenza, tanto più acuto è il suono 
Tuttavia il concetto di "altezza di un suono", come tutti i concetti che interessano la percezione, non è meramente legato alla scienza fisica: ed a noi interessa qui di prenderne in considerazione anche l'aspetto fisiologico e psichico che, unitamente a quello fisico di produzione e propagazione del suono, dà luogo alla percezione uditiva. In questa più ampia ottica, dovremmo allora prendere atto che (come è stato dimostrato) tra stimoli e reazioni non esiste in questo caso alcuna identità (tutt'al più può essere indicata una certa regolarità nel loro rapporto): frequenza e altezza del suono non sono identificabili. E si può affermare che l'altezza di un suono, sebbene fondamentalmente dipenda dalla frequenza dell'onda acustica, tuttavia è anche influenzata dalla intensità e dal timbro del suono stesso.

Il fatto che una variazione dell'intensità possa influenzare la percezione dell'altezza di un suono è legato a una particolarità della sensazione uditiva il cui effetto non è uniforme per tutte le frequenze: questo effetto è massimo per le frequenze molto alte e un po' meno marcato per quelle basse, ed è scarsamente rilevante per una larga fascia di frequenze medie.

Come abbiamo detto, l'altezza soggettiva del suono è anche influenzata dal suo timbro: nella complessa composizione di un suono, la prevalenza di certe componenti su certe altre può far gravitare la percezione in un campo di frequenze piuttosto che in un altro e, in genere, la risposta del nostro organo uditivo alla sollecitazione delle diverse frequenze non è uniforme ${ }^{3}$.

- L'INTENSITÀ è il carattere che distingue i suoni forti dai suoni deboli.

Sotto l'aspetto meramente fisico, l'intensità di un suono dipende dall'ampiezza delle vibrazioni che le particelle del mezzo nel quale l'onda si propaga compiono intorno alla loro posizione di equilibrio. Più precisamente, un suono è tanto più forte quanto maggiore è l'ampiezza delle oscillazioni.
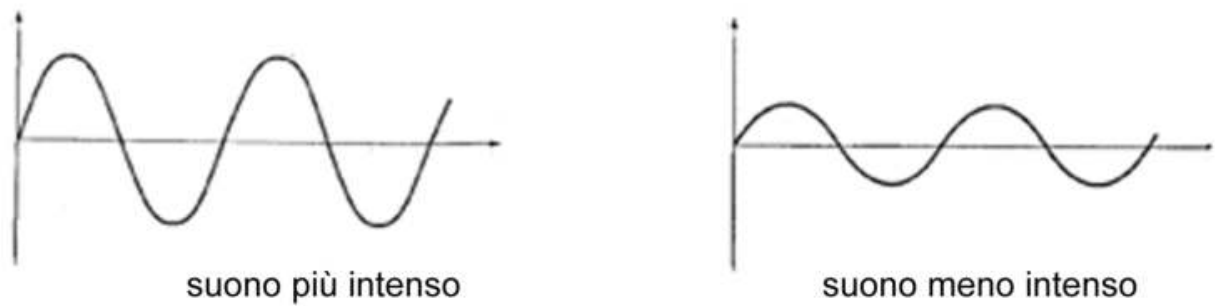

Fig. 7. L'intensità di un suono dipende dall'ampiezza dalle vibrazioni.

Da quanto abbiamo visto al punto 2, possiamo quindi dedurre che noi percepiamo un suono come più intenso o meno intenso a seconda della nostra posizione rispetto alla sorgente. Più precisamente, l'intensità è tanto maggiore quanto maggiore è l'energia trasmessa dall'onda sonora al nostro orecchio: tutto ciò che influisce su questa energia influisce sull'intensità.

\footnotetext{
${ }^{3}$ Poiché accurati studi fatti sui bambini piccoli hanno rilevato che essi si limitano per lo più a dare in questo campo un giudizio di chiaro-scuro, è stata avanzata l'ipotesi che il senso dell'altezza di un suono sia connesso anche con processi di apprendimento. Cfr. De La Motte-Haber H., 1972, Psicologia della musica, Discanto Edizioni, pag. 23.
} 
Però, come già abbiamo avuto occasione di rilevare, poiché la percezione delle caratteristiche di un suono dipende dall'azione fisiologica e psichica che le particolari vibrazioni esercitano sull'orecchio, possiamo facilmente renderci conto di come non ci sia proporzionalità fra l'energia delle vibrazioni sonore (fenomeno meramente fisico) e la sensazione che noi avvertiamo.

Ragionando in quest'ottica, dovremo anche rilevare che la percezione dell'intensità del suono è influenzata anche dalla sua altezza e dal suo timbro.

- LA DURATA di un suono si lega indissolubilmente alla "percezione del tempo" ${ }^{4}$. Ogni suono dura nel tempo, ossia si dispiega e lo si avverte lungo un arco temporale preciso, all'interno del quale è facile individuare l'iniżio e la fine del suono stesso.

- IL TIMBRO di un suono è il carattere che consente di distinguere due suoni aventi la stessa intensità e la stessa altezza, ma che provengono da due fonti diverse. L'origine del timbro ha caratteri di notevole complessità: per questa ragione ci riserviamo di ritornare su questo argomento successivamente (al punto 5), limitandoci per ora alla constatazione che suoni dal timbro diverso differiscono per la forma dell'onda (così nella fig. 8 sono rappresentati due suoni di uguale altezza e intensità, ma di timbro diverso).



A

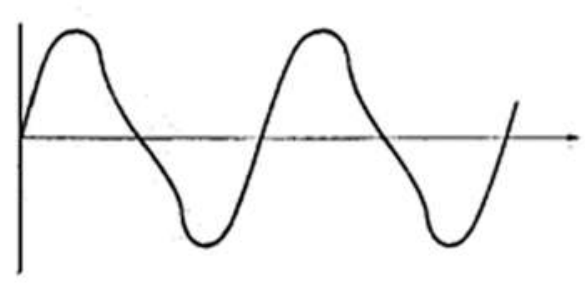

B

Fig. 8. I suoni A e B sono di uguale frequenza ed intensità ma di timbro diverso. Varia quindi la forma dell'onda

"Il suono - scriveva Shöenberg nel suo Manuale di Armonia, pubblicato nel 1911 viene percepito essenzialmente per il suo timbro, che è un vasto dominio di cui l'altezza occupa solo una piccola parte”.

Oggi sappiamo ormai con certezza che, contrariamente a quanto si è a lungo ritenuto, $i$ caratteri distintivi del suono (altezza, intensità e timbro) si influenzano vicendevolmente in modo significativo.

4 - Se indichiamo con $\mathrm{n}$ la frequenza in Hertz della nota fondamentale do, le altre note della SCALA NATURALE hanno frequenze date dalla seguente tabella:

\footnotetext{
${ }^{4}$ Cfr. a questo proposito Bertirotti A., 2002b, Musica e linguaggio: una riflessione sui risultati relativi a questo settore di ricerca recentemente pubblicati dalla New York. Academy of Sciences, in Systema Naturae, Vol. 5, in stampa; Bertirotti A., 2003 c, Il tempo nella musica. Considerazioni sull'andamento musicale, Firenze University Press, Archivio E-Print, Firenze.
} 


$\begin{array}{llllllll}\text { do } & \text { re } & \text { mi } & \text { fa } & \text { sol } & \text { la } & \text { si } & d o_{1} \\ \mathrm{n} & \frac{9}{8} \mathrm{n} & \frac{5}{4} \mathrm{n} & \frac{4}{3} \mathrm{n} & \frac{3}{2} \mathrm{n} & \frac{5}{3} \mathrm{n} & \frac{15}{8} \mathrm{n} & 2 \mathrm{n} \\ 1 & 1,125 & 1,25 & 1,333 & 1,50 & 1,666 & 1,875 & 2\end{array}$

dove nell'ultima riga sono stati scritti (in forma decimale) i rapporti di frequenza di ciascuna nota rispetto alla nota do assunta come fondamentale (indicheremo questa nota con $d o_{0}$ ): cioè il rapporto tra la frequenza di ciascuna nota e la frequenza del do centrale.

La nota $d o$, che ha frequenza doppia rispetto a $d o$, dà inizio, com'è noto, ad un'altra serie di sette note che indicheremo così:

$\begin{array}{lllllll}\text { do } & \text { re } & \text { mi } & \text { fa } & \text { sol } & & \text { si } \\ { }_{1} & & \text { si }\end{array}$

Queste sette note hanno tra loro rapporti di frequenza uguali a quelli delle precedenti. Segue un altro do che indicheremo con $d o_{2}$ e che ha frequenza doppia rispetto a quella di do (la frequenza di $d_{2}$ è dunque uguale a $4 \mathrm{n}$ ). Con $d_{0}$ ha inizio un'altra serie di sette note ... e così via.

L'intervallo di ottava è sempre uguale a 2. Così:

\begin{tabular}{|c|c|c|c|}
\hline$m i_{1}$ & $m i_{2}$ & $m i_{3}$ & $m i$ \\
\hline$\frac{5}{2} \mathrm{n}$ & $5 n$ & $10 n$ & $20 n$ \\
\hline sol, & $\mathrm{sol}_{2}$ & $\mathrm{Sol}_{3}$ & $\mathrm{Sol}_{4}$ \\
\hline $3 n$ & $6 n$ & $12 n$ & $24 n$ \\
\hline
\end{tabular}

Il diesis di una nota ha con la nota stessa un intervallo di $25 / 24$.

Il bemolle di una nota ha con la nota stessa un intervallo di 24/25.

Naturalmente abbiamo parlato sin qui della scala naturale e delle sue 17 note.

Nella SCALA TEMPERATA l'intervallo di un semitono, cioè il rapporto tra le frequenze di due qualsiasi note successive tra le 12 note esistenti, è pari a:

$$
\sqrt[12]{2}=1,06^{5}
$$

\footnotetext{
${ }^{5}$ Il numero $\sqrt[12]{2}$ è in realtà un numero che non può essere esattamente espresso in forma decimale: è infatti un numero irrazionale e le sue cifre decimali proseguono... all'infinito! Il numero 1,06 è un suo valore approssimato ai centesimi, del tutto sufficiente per una sua utilizzazione pratica. Per avere maggiori ragguagli su questo punto (e in
} 
È evidente che nella scala temperata tutti gli intervalli e quindi le frequenze delle stesse note risultano alterati rispetto a quelli della scala naturale.

Per esempio:

$$
\begin{array}{lll}
\frac{9}{8} \quad \mathrm{n}=1,125 \mathrm{n} & \text { re temperato } \\
& (1,6)^{2} \mathrm{n}=1,123 \mathrm{n}
\end{array}
$$

Fin qui abbiamo parlato di rapporti tra le frequenze delle diverse note. Se si eseguissero soltanto musiche suonate da un solo strumento, o cantate da una sola voce, i valori assoluti di queste frequenze non avrebbero importanza. Poiché si eseguono pezzi a più strumenti o a più voci, è chiaro che bisogna accordare tutti gli strumenti ad una stessa nota, che abbia una ben determinata frequenza: questa nota viene anche chiamata tono normale o diapason.

A questo scopo è stato scelto il $l a$, stabilendo che questa nota ha la frequenza di 440 vibrazioni al secondo $(440 \mathrm{~Hz})^{6}$.

Come campione internazionale si utilizza il diapason normale o corista, che è appunto un diapason che oscilla con la frequenza di $435 \mathrm{~Hz}$, depositato all'Ufficio di Pesi e Misure di Sèvres (Parigi).

Dalle tabelle riportate sopra è facile dedurre che se il la ha la frequenza di $440 \mathrm{~Hz}$ il $d o_{0}$ ha frequenza pari a

$$
\frac{3}{5} \times 440 H z=264 H z
$$

Le frequenze dei do delle diverse ottave sono allora

$\begin{array}{cccccc}d o_{3} & d o_{-2} & d o_{-1} & d o_{0} & d o_{1} & d o_{2} \\ \frac{264}{8} & \frac{264}{4} & \frac{264}{2} & 264 & 264 \times 2 & 264 \times 4\end{array}$

genere per quanto riguarda la scala naturale e la scala temperata), si veda la parte di questo capitolo, dedicata alla notazione ed ai linguaggi musicali.

${ }^{6} \mathrm{Nel}$ XVII e XVIII secolo la nota di riferimento era più alta di quella attuale (in generale la differenza rispetto a questa era all'incirca di una seconda maggiore). Tra il 1700 e il 1850, il diapason nel Teatro dell'Opera di Parigi variava tra 404 e 441 oscillazioni al secondo, ma più tardi la sua frequenza arrivò fino a 446 oscillazioni al secondo. In genere (come si è potuto verificare dall'accordatura degli organi del tempo) la frequenza del diapason manifestava una notevole tendenza a salire, e le sue variazioni producevano gravi confusioni nella prassi musicale, in particolare nella costruzione degli strumenti. Questa confusione ebbe fine solo con il Congresso di Vienna (1885) dove si stabilì di assumere come frequenza ufficiale quella del $l a_{0}$, con 435 vibrazioni al secondo. Più tardi la "International Federation of Standardizing Associations" fissò l'altezza del tono standard a 440 vibrazioni al secondo e questo valore è stato recentemente riconfermato a livello internazionale. Si potrà notare che il la scelto internazionalmente come nota di riferimento viene generalmente indicato con il $l a_{3}$ : nella notazione abituale viene infatti indicato con $d o_{0}$ il do più grave percepibile dal nostro orecchio. Noi abbiamo scelto qui, per ragioni di chiarezza espositiva, di indicare invece con do il do centrale. 
Il do di frequenza $33 \mathrm{~Hz}$ è il più grave do che il nostro orecchio può udire.

In musica vengono utilizzate sette ottave (fino al $d o_{4}$ ). La voce umana abbraccia complessivamente circa quattro ottave; ogni tipo di voce (basso, baritono, tenore, etc.) ne abbraccia circa due.

5 - Data una nota, i suoi ARMONICI sono le note dotate di frequen za multipla rispetto alla sua.

Per ragionare sugli armonici di una nota assunta come fondamentale, conviene tener conto dei rapporti di frequenza delle singole note, cioè dei rapporti tra le frequenze di ogni nota e la frequenza della nota fondamentale.

Ad esempio, i rapporti di frequenza rispetto al do centrale della scala naturale sono $i$ seguenti:

$\begin{array}{cccccccc}\text { do } & \text { re } & \text { mi } & \text { fa } & \text { sol } & \text { la } & \text { si } & \text { do } \\ 1 & \frac{9}{8} & \frac{5}{4} & \frac{4}{3} & \frac{3}{2} & \frac{5}{3} & \frac{15}{8} & 2\end{array}$

L'intervallo di terza è: $\quad \frac{5}{4}$

L'intervallo di quinta è: $\frac{3}{2}$

Se si tiene conto che l'intervallo di ottava è sempre uguale a 2 , è facile verificare che $\mathrm{i}$ primi sei armonici del do centrale sono i seguenti:

$\begin{array}{cccccc}d o_{1} & \text { sol }_{1} & \text { do } & \text { mi } & \text { sol } & d o_{3} \\ 2 & 3 & 4 & 5 & 6 & 8\end{array}$

dove sotto ad ogni armonico abbiamo scritto il relativo rapporto di frequenza rispetto al do centrale?

Ecco invece qui di seguito i primi sei armonici delle note $m_{0}$ e sol, dove sotto ad ogni armonico sono stati scritti:

- nella prima riga il relativo rapporto rispetto al do centrale.

${ }^{7}$ Il fenomeno era già conosciuto da Aristotele (384-322?), ma la prima dimostrazione sperimentale dell'esistenza dei suoni armonici si deve al fisico francese Jacques Saveur che definì scientificamente questo fenomeno nei suoi "Princìpi d'acustica e di musica ovvero sistema generale degli intervalli dei suoni, e sua applicazione a tutti i sistemi ed istrumenti di musica" (1701). Il fatto che i primi sei armonici di un suono contengano la triade maggiore sulla fondamentale fu poi posto da Jean Philippe Rameau (1683-1764, compositore e teorico musicale francese, considerato 
- nella seconda riga il relativo rapporto rispetto alla nota fondamentale (rispettivamente $m i_{0}$ e sol)

$\begin{array}{lccccc}\mathbf{m i}{ } & (\mathbf{5 / 4 )} & & & & \\ m i & s i & m i & s i & m i & s i \\ 5 / 2 & 15 / 4 & 5 & 15 / 2 & 10 & 15 \\ 2 & 3 & 4 & 6 & 8 & 12\end{array}$

$\begin{array}{lccccc}\text { sol }_{0} & (\mathbf{3} / \mathbf{2}) & & & & \\ \text { sol }_{1} & r e_{2} & \text { sol } & \text { si } & \text { re } & \text { sol }_{3} \\ 3 & 9 / 2 & 6 & 15 / 2 & 9 & 12 \\ 3 & 3 & 4 & 5 & 6 & 8\end{array}$

Uno dei risultati più importanti dell'analisi dei suoni è il seguente: pressoché ogni nota musicale, qualunque sia lo strumento che la produca (sia quindi prodotta da una corda vibrante, dalla vibrazione di una colonna d'aria o da un qualunque altro sistema vibrante), è costituita da un suono fondamentale e da un certo numero di suoni più alti, generalmente di minore intensità, noti come SUONI PARZIALI (o SOVRATONI). Il timbro del suono prodotto è in gran parte dipendente proprio dalla combinazione e dal complesso di questi suoni separati. Un suono puro, cioè un suono che sia costituito solo dalla frequenza fondamentale (un suono rappresentabile mediante una sinusoide), si ode raramente in musica.

Nella sequenza della Fig. 9 sono riportate ${ }^{8}$ le immagini reali relative a quattro vibrazioni sinusoidali costituenti la vibrazione complessa di una sorgente sonora: le immagini sono state riprese fotograficamente dallo schermo di un oscillografo elettronico (vedi alla pagina seguente).

Gli oscillogrammi a, b, c, d, riguardano rispettivamente:

a: la $1^{\text {a }}$ vibrazione armonica, ovvero la vibrazione fondamentale

b: la $2^{\mathrm{a}}$ vibrazione armonica (frequenza doppia)

c: la $4^{a}$ vibrazione armonica (frequenza quadrupla)

d: l'8 vibrazione armonica (frequenza ottupla)

Nell'oscillogramma è invece riprodotta l'azione contemporanea delle quattro vibrazioni: è la fotografia fedele della vibrazione complessa composta dalle quattro vibrazioni a, b, c, d.

I suoni usati nella pratica musicale classica (escludendo quindi i casi riguardanti la musica elettronica) sono prodotti da vibrazioni variamente complesse e proprio dalla complessa composizione di un suono dipende fondamentalmente il suo timbro (vedi il punto 3). È importante rilevare che ogni vibrazione, per quanto complessa, è sempre composta da vibrazioni semplici (di tipo sinusoidali).

il creatore della classica moderna armonia) a fondamento del suo "Trattato dell'armonia ridotta ai suoi principi naturali".

${ }^{8}$ Le immagini della Fig. n. 9 sono tratte da Righini P., op. cit., pag. 24. 




1a armonica (o fondamentale)

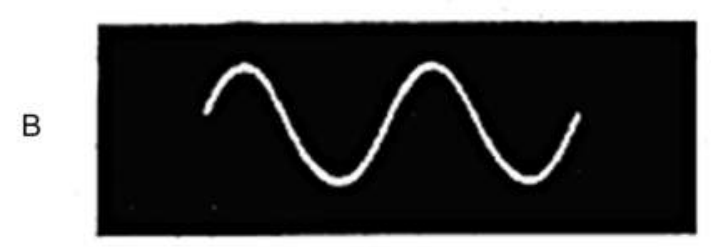

\section{$2 \mathrm{a}$ armonica}

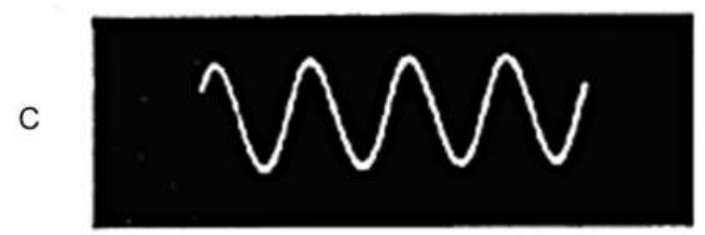

4a armonica

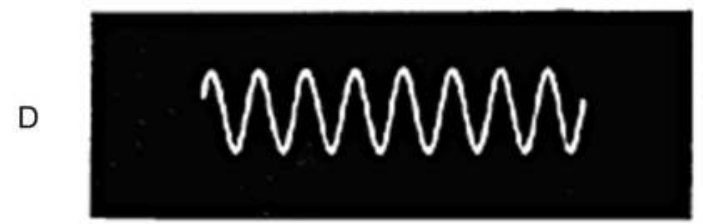

\section{$8 \mathrm{a}$ armonica}

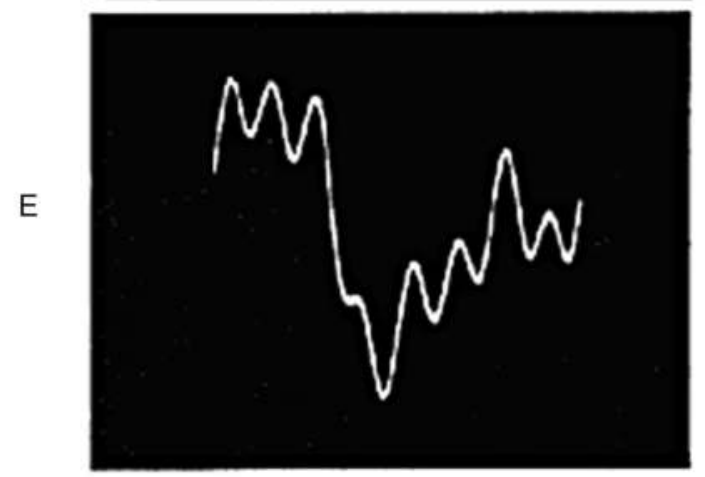

onda risultante dall'insieme dell'armoniche

Fig. 9. Esempio di nota fondamentale (A) e dei relativi tre armonici (rispettivamente $2^{\circ}, 4^{\circ}$ ed $8^{\circ}$, negli esempi B, C e D). La figura $\mathrm{E}$ rappresenta l'onda risultante dall'insieme degli armonici. 
La tecnologia dispone oggi, per la decomposizione e riproduzione dei suoni, di raffinate macchine elettroniche, la più semplice delle quali è proprio l'oscillografo a raggi catodici, dal quale sono state tratte le immagini riportate nella Fig. 9. Ci sembra interessante ricordare come, già nella metà dell'Ottocento, il fisico tedesco Helmholtz riuscisse col semplice utilizzo di opportuni risonatori a scoprire importanti caratteristiche della voce umana, portando in evidenza proprio la complessa composizione dei singoli suoni.

Facendo cantare una nota mediante le varie vocali, egli trovò che i suoni differivano per alcuni armonici e per uno o più suoni di altezza costante.

Per esempio, analizzando la vocale $u$, risultò che questa vocale presenta, col suono fondamentale, i seguenti armonici:

$$
2^{\circ} \quad 3^{\circ} \quad 4^{\circ}
$$

e il suono costante $f a_{2}$ molto debole.

La $i$ presenta invece i seguenti armonici:

$\begin{array}{lllllll}4^{\circ} & 5^{\circ} & 6^{\circ} & 7^{\circ} & 8^{\circ} & 9^{\circ} & 10^{\circ}\end{array}$

e altri anche più elevati, oltre ai suoni costanti

$$
\mathrm{fa}_{2} \quad \mathrm{e} \quad \mathrm{re}{ }_{6}
$$

Helmholtz riuscì a dimostrare sperimentalmente queste caratteristiche, utilizzando particolari risonatori capaci di rinforzare un determinato suono per simpatia e in grado quindi di rilevare l'esistenz̧a di suoni parziali: effettuando così, per la prima volta, una vera e propria analisi del suono. Egli riuscì anche a compiere, almeno in via approssimativa, l'operazione opposta all'analisi, cioè la sintesi dei suoni: combinando, con le intensità convenienti, i suoni di vari diapason, riuscì a riprodurre sensibilmente le vocali. Questo ormai non fa più effetto oggi, che siamo abituati a sentire macchine che parlano con voci umane ma, se si pensa che i lavori di Helmholtz risalgono alla metà dell'Ottocento, è possibile apprezzare in pieno il valore dell'intuizione e della capacità sperimentale di questo fisico.

Anche la classica distinzione tra suoni e rumori è legata all'analisi dei suoni.

Secondo questa concezione un suono è una nota semplice (sinusoidale) oppure una nota semplice accompagnata da alcuni suoni parziali d'intensità piccola rispetto alla sua; un rumore è invece l'insieme di numerosi suoni prodotti dalla stessa sorgente, in cui, tra il suono fondamentale (talora bassissimo) e i suoi suoni parziali, non ci siano prevalenze marcate.

Naturalmente questa distinzione non è mai stata considerata netta, nemmeno prima che $\mathrm{i}$ "rumori" entrassero a pieno diritto a far parte della musica.

A PROPOSITO DEI SUONI PARZIALI - È credenza diffusa che i suoni parziali prodotti da tutti gli strumenti musicali siano armonici, cioè che le loro frequenze siano esattamente multiple della frequenza del suono fondamentale. Questo è certamente vero per tutti gli 
strumenti a fiato e, sotto alcune condizioni, per molti degli strumenti a corda, tra cui il violino. È vero, però, in modo assai approssimativo per il pianoforte.

In realtà, se si analizzano i suoni parziali di alta frequenza di una nota del pianoforte, ci si può accorgere che questi suoni differiscono dai corrispondenti armonici: più precisamente, più alta è la frequenza dei suoni parz̧iali di ogni nota sul pianoforte, più essi si distaccano dalla semplice successione armonica.

\title{
Notazione e linguaggi musicali
}

\author{
"Profondo è il pozzo del passato. \\ Non dovremmo dirlo insondabile?" \\ T. Mann, Le storie di Giacobbe
}

\section{Il temperamento equabile}

Se ci affacciamo al "profondo pozzo", e ci sforziamo di penetrarlo col nostro sguardo alla ricerca delle origini dell'espressione musicale da parte degli uomini, ci troviamo immediatamente avvolti nell'oscurità più inquietante: questa oscurità è popolata solamente dalle voci di un dibattito che si prolunga da più di un secolo, almeno da quando Darwin ipotizzò che la musica si sia sviluppata a partire dalle vocalizzazioni dei primati sub umani, come le scimmie antropoidi.

Indipendentemente dalla generale valutazione sui principi dell'evoluzione e della selezione naturale teorizzati da Darwin, la spiegazione darwiniana dell'evoluzione della musica ha lasciato insoddisfatti una gran quantità di studiosi variamente interessati al problema specifico: questo dibattito coinvolge infatti psicologi, antropologi, etnomusicologi.

In particolare, per quanto indietro si vada nel tempo, risulta praticamente impossibile risalire alle origini della gestione da parte degli uomini delle altezze dei suoni: l'utilizzazione a fini espressivi (più o meno cosciente e razionalizzata) dei suoni musicali classificati in base al loro grado di acutezza - e cioè secondo la loro frequenza - appare infatti come un comportamento che accompagna l'uomo dagli albori del nostro mondo. Questo comportamento sembra essere un "universale assoluto": ciò significa che esistono motivi per credere che tutte le comunità umane, dalle più evolute alle più "primitive", quando si esprimono attraverso i suoni, lo facciano tenendo conto di una categoria che li distingue dal grave all'acuto.

Un tale comportamento si avvicina subito concettualmente a quello della costruzione delle scale musicali.

Una scala musicale è sostanzialmente una successione di suoni di altezza (frequenza) definita, che dividono in altrettante parti un intervallo prefissato (nel nostro caso un'ottava), ordinati generalmente in modo ascendente, dal grave all'acuto. A quanto risulta, in nessuna scala questi intervalli sono tutti uguali.

Si è visto che anche nella nostra scala naturale gli intervalli tra una nota e la sua successiva (cioè i rapporti tra le loro frequenze) sono diversi tra loro. Ragionando appunto in 
termini di frequenze, abbiamo visto che, se indichiamo con $\mathrm{n}$ la frequenza in Hertz del do centrale, le altre note della SCALA NATURALE hanno frequenze date dalla seguente tabella:

$$
\begin{array}{ccccccccc}
\text { do } & \text { re } & m i & \text { fa } & \text { sol } & \text { la } & \text { si } & \text { do } \\
\mathrm{n} & \frac{9}{8} \mathrm{n} & \frac{5}{4} \mathrm{n} & \frac{4}{3} \mathrm{n} & \frac{3}{2} \mathrm{n} & \frac{5}{3} \mathrm{n} & \frac{15}{8} \mathrm{n} & 2 \mathrm{n} \\
1 & 1,125 & 1,25 & 1,333 & 1,50 & 1,666 & 1,875 & 2
\end{array}
$$

Se cerchiamo i rapporti tra le frequenze di due note consecutive, otteniamo numeri disuguali:

- il tono intero maggiore, con il valore di $\frac{9}{8}$, tra $r e$ e do, sol e fa, si e la;

- il tono intero minore, con il valore di $\frac{10}{9}$, tra mi e re e tra la e sol;

- il semitono maggiore, con il valore $\frac{16}{9}$, tra fa e mi e tra do e si.

Tra due note che siano separate da un tono intero, ne sono state inserite altre due:

- il DIESIS: che fa 25 vibrazioni ogni 24 della precedente;

- il BEMOLLE: che fa 24 vibrazioni ogni 25 della successiva.

Esiste così a questo punto anche il semitono minore, di valore $\frac{25}{24}$, che si ha tra una nota e il proprio bemolle, o fra un diesis e la sua nota.

A questo punto se si fanno i conti', si trova che il bemolle è in tutti i casi assai più alto del diesis che lo precede. Infatti le vibrazioni corrispondenti alle note successive di un ottava sono le seguenti:

$\begin{array}{lllll}\text { do } & d o^{\#} & r e^{b} & r e & r e^{\#} \\ \mathrm{n} & 1,042 \mathrm{n} & 1,080 \mathrm{n} & 1,125 \mathrm{n} & 1,172 \mathrm{n}\end{array}$

$\begin{array}{lllll}m i^{b} & m i & f a & f a^{\#} & \text { sol } \\ 1,200 \mathrm{n} & 1,250 \mathrm{n} & 1,333 \mathrm{n} & 1,389 \mathrm{n} & 1,440 \mathrm{n}\end{array}$

$\begin{array}{lllll}\text { sol } & \text { sol } l^{\#} & \text { ld } & \text { la } & l a^{\#} \\ 1,500 \mathrm{n} & 1,563 \mathrm{n} & 1,600 \mathrm{n} & 1,666 \mathrm{n} & 1,737 \mathrm{n}\end{array}$

\footnotetext{
${ }^{9}$ Ad esempio, per calcolare la frequenza della nota $\mathrm{re}^{\#}$, basta eseguire il seguente calcolo:
}

$$
\frac{9}{8} n \times \frac{25}{24}=\frac{225}{192} n=1,042 n
$$




$\begin{array}{lll}s i & s i & d o \\ 1,800 \mathrm{n} & 1,875 \mathrm{n} & 2 \mathrm{n}\end{array}$

In tal modo si capisce così il motivo per cui non sono stati inseriti né il mi diesis né il fa bemolle: non sono stati inseriti perché non c'era posto. Sarebbero stati troppo vicini al fa: infatti questa nota è separata dal mi solo da un semitono maggiore; lo stesso vale per l'intervallo tra il si e il do successivo. L'ultima tabella esprime quindi tutte le 17 note della scala naturale, ciascuna con la propria frequenza (sempre rapportata alla frequenza della nota $d o$, indicata con $\mathrm{n})$.

Risultano evidenti le complicazioni derivanti sia dalla grande varietà degli intervalli esistenti tra nota e nota, sia dal numero troppo grande di note all'interno della scala. Verso la metà del Seicento, già s'imponeva una semplificazione: l'affermarsi degli strumenti a tastiera e le esigenze derivanti dalla nascita di una complessa e sistematica teoria dell'armonia, reclamavano la disponibilità di scale semplificate e costituite da intervalli di ampiezza costante.

Fu l'organista, teorico e compositore Andreas Werckmeister (1645-1706) che teorizzò e realizzò un nuovo sistema di intonazione e di accordatura degli strumenti a tastiera, che condusse al cosiddetto temperamento equabile .

Werckmeister non fece probabilmente che sistemare matematicamente quanto i costruttori di strumenti andavano praticando da tempo: la sua teoria faticò tuttavia a imporsi e solo anni dopo, col decisivo e geniale intervento di Johann Sebastian Bach, fu generalmente adottata.

In che cosa è consistita questa sistematizzazione? Come si è potuti passare dalla scala naturale alla scala temperata?

Questo passaggio è avvenuto attraverso due fondamentali (e coraggiose...) operazioni:

1 - È stato fuso il diesis di ogni nota col bemolle della nota successiva: le note, da 17 che erano, diventavano 12. Ma gli intervalli tra una nota e l'altra erano ancora disuguali tra loro. Allora ...

2 - Sono stati fusi (cioè resi uguali tra loro) i toni maggiori con i toni minori; e la stessa cosa è stata fatta con i semitoni maggiori e minori.

A questo punto gli intervalli sono diventati proprio tutti uguali. Ma qual è il valore di uno di questi intervalli?

Rappresentiamoli sopra una retta:

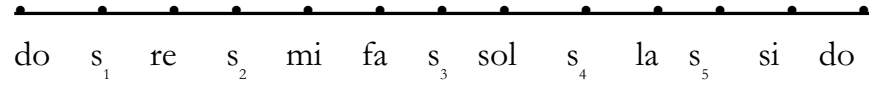

dove con $\mathrm{s}_{1}, \mathrm{~s}_{2}$, etc, sono stati indicati i successivi semitoni inseriti - ormai tutti uguali diesis e bemolle fusi: $\mathrm{i}$ tasti neri del pianoforte. 
Se indichiamo con $\mathrm{x}$ il valore dell'intervallo esistente tra due note successive della scala temperata, avremo una situazione rappresentabile in questo modo:

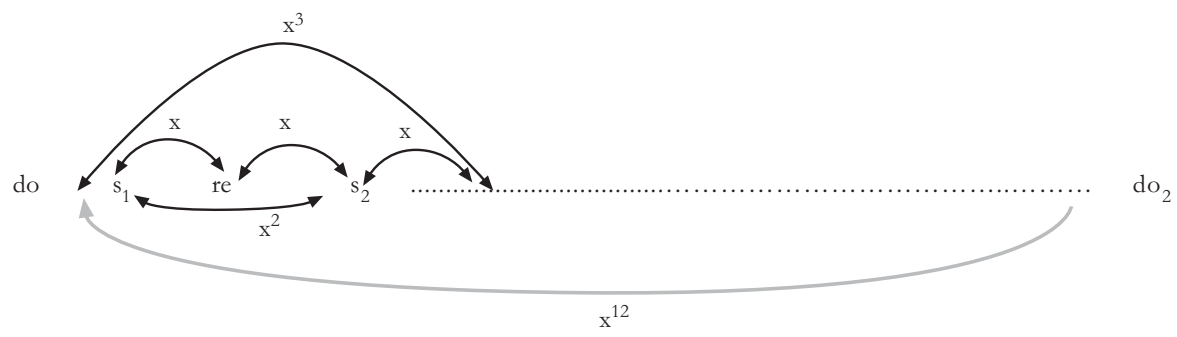

L'intervallo tra due do successivi risulta dunque uguale a $\mathrm{x}^{12}$. Ma tale intervallo, come sappiamo, è sempre uguale a 2. Risulta dunque:

$$
x^{12}=2 \quad \text { quindi } \quad \text { E) } \quad x=\sqrt[12]{2}=1,06
$$

1,06 è dunque il numero che esprime il valore del semitono temperato.

Non possiamo tuttavia esimerci dal far notare che qui c'imbattiamo in uno scoglio concettuale che farebbe inorridire i Pitagorici se avessero potuto intravederlo. Vediamo in che cosa consiste questo "monstrum".

Finora, riferendoci al valore numerico degli intervalli tra nota e nota, abbiamo sempre parlato di rapporti. Questi rapporti potevano essere espressi sotto forma di frazione (per esempio 9/8) o in forma decimale $(1,125)$ : ma sempre di numeri razionali si trattava, e ciò̀ di numeri esprimibili sotto forma di rapporto.

Nella formula E, invece, il numero 1,06 non è l'espressione, in forma decimale, di un rapporto: è l'espressione approssimata (per eccesso a meno di 1/100) di un numero irrazionale, di un numero cioè che non può essere espresso in forma di rapporto. Se ci serviamo di una calcolatrice per ricavare il valore della radice dodicesima di due, troviamo:

$$
\sqrt[12]{2}=1,0594631 \ldots \ldots
$$

Abbiamo scritto un numero con sette cifre decimali e dei puntini. Se la nostra calcolatrice ne fosse capace, potremmo calcolare tante cifre decimali quante ce ne suggerisce la nostra curiosità: i puntini stanno proprio ad indicare che il procedimento non può aver fine.

Scrivendo 1,06 nel secondo membro della uguaglianza E, abbiamo arrotondato il risultato, scegliendo un numero che non è uguale a $\sqrt[12]{2}$, ma è molto vicino a questo numero. Ci dobbiamo accontentare: $\sqrt[1]{2}$ è un numero irrazionale. 
L'intervallo di ottava (rapporto 2/1) è stato dunque suddiviso in 12 semitoni tutti uguali tra loro: solo che l'intervallo di semitono temperato non è un rapporto.

Questo fatto teoricamente rilevante (e, per la cultura greca, ideologicamente inammissibile) non ha conseguenze pratiche, e fu tranquillamente accettato dalla cultura barocca e dalle successive.

Naturalmente, se calcoliamo gli intervalli tra due note consecutive qualsiasi tra le 12 note della scala temperata, troviamo (come già abbiamo visto) che i loro valori sono tutti diversi da quelli corrispondenti della scala naturale. Tuttavia, se prendiamo in considerazione le sette note fondamentali, queste differenze sono abbastanza piccole da poterle trascurare. Quanto ai diesis, ormai unificati con i bemolle, si collocano in mezzo tra i diesis e i bemolle della scala naturale.

In conclusione, gli effetti musicali non sono sensibilmente alterati. Negli strumenti $a$ note fatte, come pianoforte, chitarra, arpa, etc., si segue sempre la scala temperata.

L'adozione del sistema equabile comporta due conseguenze pratiche di grande rilievo:

1) Poiché la scala temperata risulta divisa in 12 gradini tutti della stessa altezza, ogni intervallo musicale (costituito dalla somma di due o più intervalli temperati) risulta esattamente inserito nell'ambito dell'ottava.

2) Partendo da uno qualunque di questi gradini, possiamo costruire una scala maggiore e uscala minore: tutte le scale maggiori risultano uguali tra loro, e così tutte le scale minori.

Il Clavicembalo ben temperato di Johann Sebastian Bach (composto tra il 1722 e il 1744), forse la più monumentale e splendida opera didattica che mai sia stata scritta, rese ufficiale il temperamento equabile, imponendolo al mondo della musica con l'autorità della sua arte immensa e del suo stupefacente valore educativo.

\section{Nota sul linguaggio tonale}

Il Clavicembalo ben temperato segna la data di nascita del linguaggio tonale.

I diversi linguaggi usati nella storia della musica, in quella antica (si pensi ai diversi modi greci, o ai modi gregoriani) come in quella recente (si pensi alla diversità tra il modo maggiore e il modo minore) hanno attribuito alle diverse scale particolari valori espressivi ed affettivi $i^{10}$.

\footnotetext{
${ }^{10}$ Bernardino Streito ricorda una breve poesia di autore incerto, scritta per individuare le diverse fisionomie di ciascuno degli otto modi gregoriani, assegnando loro altrettanti "stati d'animo". Ci sembra divertente riportarla qui:

Omnibus est primus, sed alter est tristibus aptus;

tertius iratus, quartus dicitur fieri blandus;

quintum da laetis, sextum pietate probatis;

septimus est juvenum, sed postremus sapientium.

(Il primo modo è adatto a tutti, ma il secondo alle persone tristi; il terzo è adirato, ma il quarto blando, carezzevole; il quinto è per le persone allegre, il sesto per quelle pie; il settimo è proprio dei giovani, l'ottavo dei saggi.)
} 
L'attributo "tonale" indica il ruolo privilegiato che, tra le note della scala musicale, assume un suono prescelto, la tonica appunto, attorno alla quale e verso la quale gravitano gli altri suoni.

Noi non vogliamo soffermarci sui vari significati che la parola "tonalità" ha assunto nel corso della sua lunga storia nella pratica musicale europea, e nemmeno vogliamo approfondire il discorso sulla natura della musica tonale, così come ci è stata trasmessa nel corso di tre secoli.

$\mathrm{Ci}$ interessa solo ricordare come, proprio con l'affermarsi del sistema equabile, si sia venuto consolidando e affermando un sistema di funzioni tonali nel quale ad alcune note della scala viene attribuita una forte "autorità": queste note privilegiate hanno compiti di accentrazione, di repulsione e di attrazione che sono inevitabilmente destinati ad orientare ed influenzare tutto il discorso musicale. Il sistema tonale stabilisce, nell'ambito di una stessa scala, una sorta di ordine gerarchico interno. Tonica, dominante, sottodominante, sensibile ...: le note più "potenti" guidano il gioco, condizionano le mosse, costringono il musicista a percorsi obbligati, lo invitano, insinuanti o perentorie, a sottrarsi a certi "pericoli", a scegliere più convenienti soluzioni.

Tre accordi stanno a fondamento di questo sistema: le triadi di tonica, dominante e sottodominante, dove il primo accordo concede uno stato di riposo, mentre gli altri due, ponendosi in tensione col primo, determinano stati di attesa o di movimento fra le altre note.

"Se la scala è l'imitazione del suono nella dimensione orizzontale, successiva - scrive Schöenberg nella sua Harmonielebre - gli accordi ne sono l'imitazione nella sua dimensione verticale, nella simultaneità. Se la scala è l'analisi, l'accordo è la sintesi del suono" ${ }^{11}$.

Tutto il discorso musicale si sviluppa su due soli modi: il maggiore e il minore ${ }^{12}$.C’è solo da dire che, mentre il modo maggiore può (con qualche accorgimento) essere considerato come fornito a noi direttamente dalla natura, il modo minore sfugge, per quanto si sia fatto, ad una tale definizione ${ }^{13}$.

\footnotetext{
${ }^{11}$ La nostra scala maggiore - formata dai suoni do, re, mi, fa, so, la, si che stavano anche alla base dei modi greci e di quelli gregoriani - può ritenersi nata dall'imitazione della natura; intuizione e combinazione empirica hanno concorso a creare una nuova concezione della proprietà più caratteristica del suono, cioè la serie degli armonici, che noi immaginiamo disposta verticalmente come ogni sonorità simultanea: infatti essa è stata trasposta nella dimensione orizzontale, nella non contemporaneità, in sonorità successive. (...). Non siamo in grado, né ha importanza, di giudicare se i pionieri della musica abbiano trovato questa successione di suoni per mezzo dell'intuizione o della combinazione. Tuttavia, ai teorici che stabiliscono complicate teorie è lecito fare un'obiezione: quelli che l'hanno trovata erano certamente in grado di disporre, oltre che dell'istinto, anche di capacità di riflessione. Non è dunque affatto impossibile che, in questo caso, la verità sia stata trovata con la sola ragione, che cioè una parte del merito non spetti solo all'orecchio, ma anche alla capacità combinativa. Non siamo stati poi noi i primi a usare il cervello!" Schöenberg A., 1922, Manuale di armonia, Il Saggiatore, pag. 26 e 29.

${ }^{12}$ Tanto il modo maggiore quanto il modo minore sono residui dei sette modi gregoriani: il nostro modo maggiore è lo ionico degli antichi, il minore è l'eolico.

13 "Il modo minore risulta (...) un tipico prodotto artificiale e i tentativi di farlo passare per naturale non hanno senso: il modo minore è "naturale" non direttamente, ma indirettamente, come lo sono i modi antichi. Il fatto che maggiore e minore siano il risultato di un'evoluzione e che essi costituiscano una sostanziale semplificazione rispetto al sistema precedente - in quanto sono una somma contenente tutto ciò che si trovava nelle sette scale antiche e il fatto che il dualismo di questi due modi abbia la forza di un simbolo che richiama un ordinamento superiore (e questo per il fatto che esso ricorda la dualità dei generi e che delimita a piacimento le sfere d'espressione), potrebbe
} 
Osserviamo ancora che il "temperamento equabile" offre la possibilità di identificare una stessa frequenza (uno stesso tasto di pianoforte) con note di nome diverso: lo scambio enarmonico conferisce ad ogni grado della scala una polivalenza di significati e funzioni che arricchisce significativamente le possibilità di articolazione del discorso musicale.

Il linguaggio tonale, così come Bach lo costruì nella prima metà del Settecento, e come poi ci è stato rigorosamente trasmesso attraverso la sacralizzazione fatta prima dagli autori classici e poi da quelli romantici (che l'adottarono in toto come fondamento e legittimazione del loro paesaggio sonoro), risulta quindi caratterizzato da un'estrema flessibilità e da una feconda ricchezza di possibilità sintattiche; ma, nello stesso tempo, risulta carico di lacci e lacciuoli, affollato di regole prescrittive (alle quali veniva quasi attribuito un contenuto etico), di convenzioni e leggi ed anche di ambiguità gerarchiche nel suo lessico e nella sua grammatica.

La vitalità creativa e, più in generale, la coscienza percettiva e musicale dei secoli diciottesimo, diciannovesimo e ventesimo hanno assorbito l'etica tonale e ne hanno fatto il proprio mondo: e questo mondo si è progressivamente arricchito ed ha accumulato tesori di sapienza e di arte: i trattati di armonia, di contrappunto, di composizione, di teoria della forma, ed una ricchezza di produzione musicale che continua (nonostante il radicale cambiamento dei tempi) ad affascinare e a raccogliere intorno a sé studiosi ed appassionati, stanno a testimoniare l'evoluzione e il successo di questo linguaggio. E, probabilmente, anche a giustificare il suo rapido invecchiamento e la rivoluzione linguistica che, all'inizio di questo secolo, diede vita ad una Nuova Musica.

Chissà se tutto questo è sufficiente anche a giustificare l'esplosione che negli ultimi decenni ha mandato in frantumi le residue consolatorie certezze che ancora derivavano dall'esistenza di "tavole della legge" e che, in un modo o nell'altro, guidavano la fantasia creativa dei compositori e la coscienza percettiva degli ascoltatori?

Ma a questo punto il discorso si complica, coinvolgendo considerazioni storiche, culturali, sociologiche e psicologiche; e noi qui lo lasciamo, riservandoci solamente di accennare a quella rivoluzione linguistica che segnò l'inizio della fine dell'impero della tonalità.

\section{Nota sul linguaggio seriale}

"Metodo di composizione con dodici note non imparentate tra loro": così Arnold Schöenberg (1939) definisce il suo procedimento compositivo, che venne poi generalmente conosciuto col nome di sistema dodecafonico.

Specificando ancora meglio, egli lo definisce altrove con queste parole:

"Metodo di Composizione con Dodici Note, basato sull'Emancipazione della Dissonanza, che consiste nell'equipollenza fra idea di dissonanza e idea di consonanza".

incoraggiare l'eresia che questi due modi siano l'unico vero fenomeno naturale in musica, quello definitivo e destinato a perdurare, e che essi soddisfino pienamente la volontà della natura. Per me le cose stanno diversamente: alla volontà della natura ci si è avvicinati, questo si, ma se ne è ancora lontani; gli angeli, che sono la nostra superiore natura, non hanno sesso, e lo spirito non conosce capricci.”. Schöenberg A., ibidem, 119. 
Il metodo ideato da Schöenberg (con un travaglio che durò lunghi anni) fornisce il primo uso sistematico della serie, dando vita ad una musica basata su una successione fondamentale di dodici suoni, senza ripetizione né raddoppio all'ottava, disposti dal compositore secondo un ordine che determinerà l'ulteriore sviluppo.

Siamo nel 1909 quando Schöenberg pubblica i Klavierstucke Op. 11 (Tre Pezzi per pianoforte), uno dei primi capolavori della libera atonalità, che segna l'apertura di uno strappo destinato ad allargarsi sempre più tra il sistema tonale e la musica del Novecento. Ma la prima integrale applicazione del sistema dodecafonico compare nel 1923 con la Suite Op. 25 per pianoforte. La gestazione, come si vede, non è stata breve né poco maturata.

Siamo ben lontani dal voler tentare una descrizione del metodo Schöenberghiano: ciò che qui interessa è solo di mettere in evidenza come, pur rimanendo sinora all'interno di una stessa notazione, quella fornita dalla scala cromatica, il paesaggio sonoro che veniva offrendosi all'ascoltatore venisse completamente trasformato.

Per Schöenberg non esiste un concetto fondamentale di dissonanza: esistono solo stati di consonanza più o meno lontani. Egli abolisce quindi ogni gerarchia tra i dodici suoni della scala cromatica, accordando a ciascuno di essi pari dignità armonica. Spariscono così i privilegi, i rimandi, le relazioni a distanza: tonica, dominante, sensibile ... sono parole che scompaiono dal vocabolario musicale.

Ma lo spirito costruttivo di Schöenberg non poteva essere interessato ad un atteggiamento puramente negativo. La parola "atonalità" rende conto solo di un aspetto della sua teoria: e infatti a lui questa parola non piaceva.

Dopo aver "composto" il suo Harmonielehre, un testo ancor oggi fondamentale per chi voglia affrontare lo studio dell'armonia in maniera non schematica e ripetitiva ed aprire il suo sguardo all'armonia atonale, Schöenberg formula il suo “ordine nuovo". Perché certamente di un ordine si tratta, e di un ordine assolutamente rigoroso. In uno dei suoi scritti polemici, egli così apostrofa "quei dilettanti che credono che comporre con dodici note non significhi altro che usare sempre la forma-base e le sue inversioni. Di fatto il significato di questa regola dovrebbe essere espresso in altro modo. Dovrebbe essere: nessuna delle dodici note deve apparire al di fuori dell'ordine stabilito dalla forma-base o delle sue forme derivate. Ma pensare che l'obbedienza a questa regola produca una composizione è puerile, dilettantesco, da incompetente né più né meno che supporre che evitare altre proibizioni sia sufficiente per creare musica. Ad esempio, evitare quinte ed ottave parallele. Queste regole sono solo restrittive, non creative. Bisogna essere in grado di produrre musica malgrado queste severe restrizioni” ${ }^{\prime 1}$.

Accenniamo brevemente come, nelle composizioni aderenti al sistema dodecafonico schöenberghiano, un "tema” può essere composto da parecchi frammenti melodici, ciascuno appartenente ad una delle quarantotto formule disponibili ${ }^{15}$; ogni frammento può

\footnotetext{
${ }^{14}$ Cfr. Schöenberg A., Mann T., 1950, A proposito del Doctor Faustus, Rosellina Archinto, Milano, pag. 55.

${ }^{15} \mathrm{Nel}$ sistema dodecafonico, ogni serie si può presentare sotto quattro forme, secondo il principio impiegato negli stili tradizionali d'imitazione contrappuntistica: forma originale, forma retrograda o cancrizzante, inversione dell'originale (ove si ha l'inversione degli intervalli), retrogrado dell'inversione. Ciascuna di queste forme ammette una trasposizione su ognuno dei dodici gradi della scala cromatica, con un totale quindi di quarantotto versioni disponibili di una stessa serie.
} 
poi essere armonizzato con suoni complementari tolti ad un'altra versione della serie generatrice. Ulteriori complicazioni possono poi venir inserite nella composizione (scrittura a canone, variazioni ritmiche, ecc.). Alla base di tutto sta comunque la prescrizione che nessuna nota deve più occupare una posizione privilegiata. "Una sia pur leggera reminiscenza della vecchia armonia risulterebbe fastidiosa, poiché creerebbe inevitabilmente false speranze riguardo alle conseguenze e alle continuazioni. L'uso di una tonica è deludente se non è basato sul fatto di reggere tutte le relazioni della tonalità. Allo stesso modo, l'impiego di più di una serie è da escludere, poiché in ogni serie successiva uno o più suoni verrebbero ripetuti troppo presto. Un tale uso di più serie farebbe correre il rischio che un suono ripetuto possa essere interpretato come una tonica. In genere, l'effetto d'unità ne risulterebbe diminuito" 16 .

Schöenberg (il quale non deve certo la sua fama ad una carattere particolarmente conciliante...) non riteneva tuttavia che fosse ormai venuto il tempo di bandire dal mondo compositivo il sistema tonale. La sua opposizione più totale si manifestava però contro coloro che "racimolano dissonanze, volendo così passare per moderni, ma non hanno il coraggio di trarne le conseguenze".

A chi, riferendosi alla voluta razionalità della sua ricerca linguistica, lo accusa di essere un compositore "intellettuale", lui risponde tagliando corto: "Preferisco comporre da intellettuale che da imbecille!".

Un nuovo ordine, dunque: e, si potrebbe forse aggiungere, un nuovo programma etico!

L'opera di Schöenberg fu completata e sviluppata dai più grandi dei suoi allievi: Alban Berg e Anton Webern.

Abbiamo ritenuto necessario fare qualche particolare accenno alla dodecafonia seriale, perché ci sembra abbia rappresentato il radicale inizio di un'avventura linguistica destinata a svilupparsi in modi probabilmente del tutto impensati da coloro stessi che ne furono i principali protagonisti, travolgendo, in definitiva, tutti gli steccati esistenti.

Naturalmente l'opera di Schöenberg e della sua scuola non esaurisce il ruolo svolto dalla "serie" nel variegato panorama compositivo dei primi decenni del Novecento. È forse il caso di fare qui un cenno almeno ai casi di organizzazione seriale in musiche diatoniche (come in Strawinskij e Malipiero) e ricordare che, nelle scuole d'avanguardia dei primi anni Cinquanta, il principio della serializzazione è stato esteso anche a tutti i parametri sonori, come il timbro, la dinamica, l'attacco, l'intensità e la durata.

Ciò che è avvenuto in seguito, l'evoluzione successiva in forme molteplici, e spesso anarchiche, dei linguaggi della musica non può certo far parte di questa breve informazione.

\footnotetext{
${ }^{16}$ Schöenberg A., La composizione con dodici suoni, conferenza del 1939.
} 



\title{
2. Considerazioni sulle origini e l'evoluzione del suono e della musica
}

\author{
"Dunque senza la musica nessuna disciplina può esse- \\ re perfetta, perché senza di essa non esiste nulla. La \\ musica muove i sentimenti e modula le emozioni. La \\ musica consola la mente nel sopportare le tribolazioni \\ e la modulazione della voce conforta la fatica di ogni \\ lavoro. ... ogni parola che pronunciamo, ogni pulsazio- \\ ne delle nostre vene è legata dal ritmo musicale ai \\ poteri dell'armonia". \\ Isidoro di Siviglia, vescovo della città, \\ in "Cosa può fare la musica", \\ (627 ca. d.C.)
}

\section{Premessa}

Sino alla metà del secolo scorso, parlare di musica in Occidente equivaleva a parlare di musica tonale: riteniamo addirittura che ancor oggi le cose siano rimaste tali per un numero non trascurabile di persone. Tuttavia, la varietà di musica presente sul nostro pianeta è incredibilmente grande. Con la scoperta delle grandi culture musicali dell'Asia (specialmente dell'India) e dell'Africa, si è imparato ad apprezzare l'importanza e la ricchezza di questa varietà: alcuni studiosi hanno allora cominciato a valutare con imbarazzo l'etnocentrismo convinto che stava caratterizzando la nostra cultura. Con lo sviluppo dell'etnomusicologia si è infine scoperto che la musica occidentale non era affatto l'unica musica elaborata esistente e che certe musiche erano state considerate primitive solo perché non erano state adeguatamente analizzate e comprese. Gli etnomusicologi hanno dunque indirizzato il loro lavoro verso lo studio e la registrazione sistematica della musica reperibile nelle culture diverse dalla loro: questa impostazione classificatoria ${ }^{17}$ è stata sicuramente il

${ }^{17}$ Il metodo di classificazione etnomusicologica di Erich von Horbostel e Curt Sachs del 1914 è considerato, ancora oggi, il migliore. “(...) si basa sulle caratteristiche generative del suono e divide gli strumenti musicali (anche quelli della musica classica occidentale) in quattro classi fondamentali: idiofoni, membranofoni, aerofoni e cordofoni, ciascuna delle quali è, a sua volta, divisa in gruppi e sottogruppi. (...) [Appartengono al gruppo degli idiofoni] tutti gli strumenti che pur non avendo un dispositivo (corde, membrane, fori) emettono comunque suoni per percussione, $(\ldots)$ concussione, (...) sfregamento, (...) scotimento (...) o pizzicamento (...). Nella classe dei membranofoni si raccolgono tutti gli strumenti che producono suono utilizzando la vibrazione di una membrana in tensione posta su una cavità. Si dividono in quattro grandi gruppi: tamburi a percussione, tamburi a pizzico, (...) tamburi a frizione, e strumenti che $(. .$.$) deformano la voce umana o altri suoni. La terza classe, gli aerofoni, comprende tutti gli strumenti che$ generano suono tramite la vibrazione dell'aria. (...) Infine, la classe dei cordofoni raccoglie tutti quegli strumenti nei quali una più corde $(. .$.$) sono tese tra punti fissi del corpo dello strumento e emettono un suono utilizzandone la$ vibrazione." Giuntini G., 2001, Gli utensili del musicista, in Suoni della terra. Forme musicali, Catalogo della mostra, Fondazione Carlo Marchi, Firenze, pagg. 20-23. 
primo passo per la comprensione delle musiche altre ed ha anche comprensibilmente reso possibile un avvicinamento alle corrispondenti culture. L'interesse che l'etnomusicologia pone nella ricerca di "esempi di comportamento musicale" nasce infatti dalla possibilità che, nel comportamento musicale, risposte simili vengano date in relazione a situazioni socioculturali simili1. " Impostando così il problema, i suoni musicali di una cultura vengono classificati, per esempio, nei termini degli strumenti che vengono utilizzati, delle forme tipiche che si incontrano, delle scale e dei sistemi d'intonazione usati, dei contesti sociali in cui si produce musica, e così via. Utilizzando queste informazioni, è possibile costruire una "mappa mondiale" comparativa della musica, raggruppando le culture che appaiono più simili sotto quest'aspetto" 19 .

La definizione dei prodotti musicali si può sviluppare in sintonia con le definizioni di insieme in matematica ${ }^{20}$. Per stilare un inventario delle pratiche che la teoria a monte considera musicali (applicazione del metodo in estensione), l'etnomusicologo decide se una pratica può rientrare fra gli oggetti musicali della sua ricerca; poi analizza, sulla base dell'inventario stilato, le pratiche inserite, comparandole per individuarne i tratti comuni (applicazione del metodo in comprensione).

A questo proposito, dobbiamo porci due domande. La prima: come fa l'etnomusicologo a decidere che quella pratica musicale rientra nel suo settore di studi? La seconda: su che cosa si fonda la somiglianza tra due pratiche musicali?

Poiché le denominazioni locali non possono essere d'aiuto (in molte lingue africane e amerinde non esiste neppure la parola "musica"), la risposta alla prima domanda sembra non possa essere che questa: l'etnomusicologo individua e descrive la cultura studiata in base alle proprie categorie musicali (canto liturgico, canto profano, musica strumentale, recitazione, etc.), e questo lo porta ad assimilare le pratiche studiate alle pratiche della sua cultura $^{21}$. Sembra che non si possa sfuggire ad una forma di etnocentrismo metodologi$\mathrm{CO}^{22}$.

La ricerca di una risposta alla seconda domanda apre problemi ancora maggiori.

\footnotetext{
${ }^{18}$ Cfr. Delalande F., 1993a, Le condotte musicali. Comportamenti e motivazioni del fare musica e ascoltare musica, Editrice Clueb, Bologna.

${ }^{19}$ Cfr. Sloboda John. A., 1985, The Musical Mind. The Cognitive Psychology of Music. Oxford University Press, trad. it. La mente musicale. Psicologia cognitiva della musica, Società editrice il Mulino, Bologna, pag. 369.

${ }^{20}$ In matematica un insieme può essere definito in estensione, cioè indicando la lista degli elementi che compongono l'insieme, oppure in comprensione, cioè indicando una proprietà che permetta di decidere se quell'elemento appartiene o no all'insieme.

${ }^{21}$ A questo proposito F. Delalande scrive: “Andiamoci piano, comunque - a ridere dell'etnocentrismo un po' gretto dei nostri predecessori, perché, se fortunatamente l'abbiamo superato, è stato solo per trovarne un altro, a un altro livello. Più simpatico, è vero, in quanto privo di quel tono di disprezzo, ma non superabile, in quanto costitutivo del sapere etnologico" Delalande F., op. cit. pag. 39.

22 Molti autori ammettono come inevitabile il ricorso ad una intuizione etnocentrica di che cosa è la musica. "Potrei dirmi che quei fenomeni fuori della mia cultura più immediata, ai quali ora attacco l'etichetta "musica" perché li riconosco e identifico come musica, sono identificati così solo perché, giustamente o erroneamente, mi sembra che assomiglino ai fenomeni che io sono abituato a chiamare musica nella mia terra natale. Sono abituato a considerare un gruppo (più o meno) definito di fenomeni come musica: questo gruppo abbraccia un numero di proprietà differenti che non posso definire con chiarezza, eppure non ho dubbi che essi appartengano a questo gruppo "musica". Vedi in Nettl B., 1984, The Study of Ethnomusicology, University of Illinois Press, pag. 25.
} 
Il concetto di musica può essere considerato a due diversi livelli: come classe di oggetti sonori e come classe di pratiche sociali. "Gli oggetti sonori sono i prodotti sonori delle attività considerate musicali, e lo studio comparativo a questo livello consiste nel cercare se questi prodotti hanno in comune degli elementi strutturali, come le scale, i ritmi, etc.

Le pratiche sociali consistono in gente, azioni e suoni, $\mathrm{o}$, più precisamente, consistono nelle azioni che la gente compie rispetto a dei suoni; lo studio non si limita più agli oggetti sonori, ma include le azioni che questi provocano. Il problema è allora di sapere se fare (o capire) la musica consiste in attività che, in differenti continenti, si assomigliano"23.

Ci si riferisce qui in parte al concetto di musica come classe di oggetti sonori e classe di pratiche sociali, tenendo però presente che qualsiasi oggetto sonoro ha un soggetto attivante naturale e induce quindi sempre una pratica sociale. Il punto di vista qui adottato non è dunque tanto etnomusicologico, quanto piuttosto etno-antropologico: i suoni che si strutturano in musica entrano infatti a far parte, fin dai primordi, di quella memoria della specie dalla quale deriva il nostro attuale rapporto con i suoni.

La nostra attenzione si concentrerà prima nel riconoscimento di una relazione circolare suono-uomo-suono che accompagna, presumibilmente sin dalle origini, l'evoluzione della specie, per prendere poi in considerazione il passaggio dalla pratica del suono alla sua notazione e, in particolare, alla notazione musicale considerata da due angolazioni: come simbolizzazione nella prassi esecutiva della condotta sonora e come strutturazione grammaticale e sintattica del materiale sonoro, portatrice di vantaggi e svantaggi per l'evoluzione della musica stessa.

La notazione musicale non presenta ovviamente in sé elementi chiari di riferimento alle pratiche sociali che ad essa sono sottese. Queste pratiche sono tuttavia caratterizzate da atteggiamenti e condotte che potremmo definire musicali, perché mettono in scena un rapporto (legittimato dalla cultura) tra l'uomo, che le esegue o ne fruisce, e il mondo dei suoni. Si è autorizzati a supporre che l'aspetto simbolico presente nella musica consista nella possibilità di ripercorrere, durante l'esecuzione, le pratiche socio-musicali sedimentate nella memoria collettiva e frutto dell'evoluzione della specie. La musica (nella sua strutturazione notazionale), eseguita e fruita, diventa così la messa in scena delle pratiche musicali che la definiscono.

Il termine cultura è forse il più ricordato dagli autori che si occupano del fattore umano e dunque anche dai musicologi in questo testo. Psicologi, sociologi, letterati, criminologi, urbanisti, etc. si riferiscono alla cultura quando trattano temi umani del proprio ambito di competenza.

Per converso, tra tutte le fenomenologie di base relative all'uomo e alla sua mente, il termine ed il concetto di cultura risultano non soltanto meno descritti dai non antropologi, ma sovente vengono dati per conosciuti. In pratica, tutti ne parlano, e tutti sanno che un universo di fenomeni detto cultura fa parte della componente umana in qualunque società,

\footnotetext{
${ }^{23}$ Delalande F., 1993b, Che cosa è la musica?, in Il senso della musica. Semiologia della musica, (a cura di) Ferrari Franca e Stefani Gino, dispensa del Corso quadriennale di Musicoterapia, Centro di Educazione Permanente, La Cittadella, Assisi, pag. 22-23.
} 
secondo la tradizione, accanto ai fenomeni sociali e a quelli psicologici. Sono però ben rari quelli che si preoccupano del rapporto tra la cultura e il loro oggetto, vale a dire la mente, la società stessa, l'arte, etc. Nel nostro del rapporto tra la musica e la cultura. Non basta affermare che il rapporto della cultura con l'autore dell'opera o con il fruitore di essa costituisce una relazione fondamentale, se poi non se ne specifica il senso o come la relazione si attivi.

A partire già dal 1700, della cultura si sono date molte definizioni. Oggi, dal punto di vista cognitivo, è definita come un sistema di norme e atteggiamenti mentali che caratterizzano il modo di vivere, di pensare la realtà e di reagire ad essa ed ai suoi stimoli, e dunque il mondo della vita in ogni società. Ogni società sviluppa la sua cultura sulla base di una struttura di categorie mentali universali, individuate in quattordici, che stanno a fondamento degli atteggiamenti mentali. Esse sono in buona parte le categorie del meta-mentale che già Aristotele aveva descritto nell'Organon ${ }^{24}$. Questi atteggiamenti mentali sono innati $^{25}$, vale a dire in ogni società sono ontofilogenetici; essi regolano la formazione del patrimonio dei contenuti mentali e lo sviluppo delle regole del rapporto dell'uomo con il suo mondo, vale a dire appunto la sua cultura.

Si comprende come questo meccanismo, altamente complesso, giochi appunto un ruolo determinante anche nella costruzione dell'opera d'arte o musicale e tanto più nel suo consumo.

Gavino Musio, come antropologo cognitivo, ha espresso in sintesi i concetti di base di questo rapporto tra opera, comunicazione, fruizione di essa e cultura, anche se la complessità dei processi relativi, detti appunto cognitivi, richiederebbe una trattazione comprensibilmente di notevole estensione ${ }^{26}$.

\section{Una relazione circolare suono-uomo-suono}

Nel libro della Genesi troviamo all'inizio questa frase: "Iddio disse: «Sia la luce»: e la luce fu" 27 .

Nella solenne enunciazione biblica Dio crea il cielo e la terra in un mondo dominato dal silenzio. Su questo mondo diffonde la sua voce ed è proprio la sua voce l'atto creativo della luce. Il suono - la voce di Dio - esiste dunque, nella tradizione biblica, come prerogativa divina. Il suono non viene creato: il suono è. Il suono esiste in quanto esiste Dio, creatore del cielo e della terra.

Ritroviamo questo concetto in tutte le convinzioni cosmogoniche presenti sulla terra. "Tutte le volte che la genesi del mondo è descritta con sufficiente precisione, un elemen-

\footnotetext{
${ }^{24}$ Cfr. Lelli G. (a cura di), 1955, Aristotele, Organon, Einaudi, Torino, vol. I.

${ }^{25}$ Klukhohn Fl. et alii, 1960, variations in Value Orientations. A Theory tested in Five Cultures, Cambridge.

${ }^{26}$ Si veda Musio G., 1989 (5a ed.), Antropologia e mondo moderno, Franco Angeli, Milano, Musio G., 1995, La mente culturale, Univ. di Firenze, Firenze, Lelli G. (a cura di), 1955, op. cit.. Per le matrici antropologiche di questo approccio, si veda Klukhohn Fl. et alii, 1960, Variations in Value Orientations. A Theory tested in Five Cultures, Cambridge. Per le matrici illuministiche della scienza della cultura, cfr. anche Del Carlo I., 2001, Il concetto di Kultur nel pensiero storicistico tedesco, tesi di laurea, Univ. di Firenze, Fac. di Scienze della formazione, relatore Musio G.

${ }^{27}$ Genesi, 1, 1-3.
} 
to acustico interviene nel momento decisivo dell'azione. Nell'istante in cui un dio manifesta la volontà di creare se stesso o un altro dio, di far apparire il cielo e la terra oppure l'uomo, egli emette un suono. Espira, sospira, parla, canta, grida, urla, tossisce, espettora, singhiozza, vomita, tuona, oppure suona uno strumento musicale" ${ }^{28}$. Il creatore, quindi, accompagna il suo atto primigenio con l'emissione di un suono e, alcune volte, è un suono fragoroso come quello di un tuono ${ }^{29}$. Vi sono anche tradizioni che oltrepassano la "fisicità" pura e semplice del suono e attribuiscono al canto la forza generatrice.

Poiché comunque il creatore è suono o canto (suono articolato melodicamente), il mondo cui concede la vita non può che essere sostanzialmente sonoro e dall'ascolto dipende la sopravvivenza. Ascoltare, domandare e rispondere appaiono così, sia nella ricostruzione biblica sia nelle altre concezioni cosmogoniche, come le forme filogenetiche più antiche delle nostre azioni attuali: il rapporto circolare suono-uomo-suono si rappresenta come motore fondamentale dello sviluppo della specie.

È oramai convinzione comune che l'evoluzione umana, e nella fattispecie il salto dall'uomo di Neandertal al sapiens, sia dipesa anche dallo sviluppo dell'apparato telemetrico (la vista e l'udito). Il senso della vista risulta, peraltro, associato a quello dell'udito nella maggior parte delle cosmogonie, se è vero che, in un gran numero di miti, i primi canti della creazione portarono il chiarore o l'aurora ${ }^{30}$. Secondo André Leroi-Gourhan ${ }^{31}$, lo sviluppo della specie uomo, che sicuramente è in larga parte legato alle sue capacità uditive e di linguaggio, è stato determinato da alcune sue strutture somatiche e in particolare dalle peculiarità della sua struttura cerebrale ${ }^{32}$.

La fisiologia dei nostri antenati, evolutisi dai pesci, alle scimmie sino all'Homo, dimostra altresì la presenza della capacità auto-osservativa come pre-requisito evolutivo: l'osservazione degli arti posteriori ha permesso l'assunzione della postura eretta e l'osservazione del possibile utilizzo degli arti anteriori ha determinato la nascita di nuove strategie adattative. Una volta raggiunta la posizione eretta, l'uomo ha potuto ampliare il suo orizzonte ed

\footnotetext{
${ }^{28}$ Schneider M., 1960, Le róle de la musique dans la mythologie et les rites des civilisations non européennes, Éditions Gallimard, Paris, trad. it. 1992, La musica primitiva, Adelphi Editore, Milano, pag. 13.

29 "Tale assimilazione è sicuramente molto antica: la troviamo infatti già nella mitologia di popoli primitivi come i Californiani, gli Aranda dell'Australia, i Samoiedi e i Coriaki dell'Asia settentrionale. (...) L'idea del mondo generato dal canto deve avere un'origine molto remota. A dimostrarlo basterebbe la sua diffusione, ma appare antichissima (...). Le civiltà tecnicamente più progredite ci mostrano spesso il creatore come un vasaio, un falegname o uno scultore il quale, dopo aver foggiato i corpi, comunica loro la vita mediante un grido, un'espirazione o la saliva." Schneider, M., ibidem, pagg. 15-17.

30 Schneider M., ibidem.

${ }^{31}$ Leroi-Gourhan A., 1977, Il gesto e la parola, Einaudi, Torino.

${ }^{32}$ In ottica cognitivista è interessante il modello di Jerry Fodor che si inserisce in una teoria più generale e modulare della mente. Secondo Fodor, nella nostra mente esisterebbero due sistemi: sistemi di imput agenti come trasduttori sensoriali e linguistici e sistemi centrali che svolgono il ruolo di interfacciamento e di formazione-fissazione della credenza. Secondo Fodor, i sistemi analitici di imput sono caratterizzati dalla specificità di dominio, ossia dalla obbligatorietà delle loro operazioni (nel caso delle percezioni sonore, la specificità di dominio è quella uditiva) e dalla estrema rapidità delle informazioni sensoriali. Sul piano strettamente musicale, Fodor ipotizza dei veri e propri sistemi computazionali "che rivelano la struttura melodica o ritmica dello stimolo acustico." Cfr. Fodor J., 1983, The Modularity of Mind, The MIT Press, Cambridge Massachusetts, trad. it. 1988, La mente modulare, Il Mulino, Bologna.
} 
identificare la provenienza dei suoni circostanti. La specializzazione dell'udito, associata ad altri sensi, ha permesso la nascita di un primo sé sensomotorio ${ }^{33}$ che, a sua volta, ha favorito lo sviluppo della direzionalità della fonazione ${ }^{34}$. Ha così avuto inizio il processo di auto-riconoscimento attraverso l'identificazione di altri esseri come lui.

Riteniamo che proprio nel processo di autoriconoscimento risieda la nascita filogenetica di ciò che viene definita "personalità". Ogni elemento naturale - sia questo un oggetto o un gruppo di oggetti, essere umani ed animali, oppure eventi fisici esterni - si trova, infatti, ad interagire costantemente con l'uomo stimolando le sue domande e chiedendogli risposte continue. Nell'atto del rispondere, l'uomo impara a distinguersi come entità autonoma assumendo atteggiamenti di autocoscienza, i quali permettono in lui lo sviluppo di un sé, ossia della personalità. Tra i tanti eventi fisici naturali che hanno stimolato questo atteggiamento si sono sicuramente imposti i suoni della natura, vale a dire quell'insieme di domande sonore che caratterizzano il ritmo della vita.

Il contatto sensoriale, che si stabilisce durante l'ascolto di suoni, è di tipo fisiologicoreattivo e tale da innescare meccanismi attentivi focalizzati ad individuare le caratteristiche del suono stesso e la sua fonte. La "teoria del contatto" sembra la più convincente all'in-

\footnotetext{
${ }^{33}$ Il concetto di "sé sensomotorio" è stato introdotto in sede psico-pedagogica da Jean Piaget. L'unità cognitiva centrale della teoria piagetiana è l'operażone, dove s'intende con questo termine quella particolare regola dinamica che deriva dall'interazione del bambino con gli oggetti del reale. Questa regola contiene in sé le conoscenze che possono tradursi, secondo le circostanze, in azioni o pensieri: un'operazione è, ad esempio, la capacità di programmare mentalmente una serie di azioni (o una sequenza di suoni) per poi riuscire a ripercorrere a ritroso ogni tappa della sequenza programmata. È convinzione di alcuni studiosi che lo sviluppo cognitivo ontogenetico ripercorra gli stessi stadi dello sviluppo filogenetico. Si tratta della "legge di Haeckel, secondo la quale l'ontogenesi (cioè l'evoluzione dell'individuo) ricapitola la filogenesi (cioè l'evoluzione della specie): per Haeckel, infatti, l'embrione umano presenta via via caratteristiche simili a quelle rispettivamente dei pesci, degli anfibi, dei rettili e dei mammiferi inferiori." Cfr. Balbi Renato e Rosellina, 1982, Lungo viaggio al centro del cervello, Edizione Club su licenza G. Laterza, Bari, pagg. 8-9. In altre parole, a partire dalla vita intrauterina il cervello di ciascuno passa attraverso gli stessi stadi percorsi dall'antenato dell'uomo. L'encefalo di un feto umano di tre mesi, ad esempio, è simile a quello di un marsupiale mentre, tra il terzo e il quarto mese, acquista le caratteristiche dell'encefalo dei roditori, poi dei carnivori e quindi di quello dei primati. Nessuno di questi livelli cerebrali viene distrutto. Dal punto di vista della psicologia evolutiva, si postula l'esistenza di stadi evolutivi che caratterizzano la formazione di domini cognitivi. Ci si riferisce alla teoria piagetiana, in cui si individuano quattro stadi principali: a) lo stadio sensomotorio, (dalla nascita sino a diciotto mesi) durante il quale la realtà esterna viene percepita attraverso i sensi ed il movimento; b) lo stadio preoperativo (da diciotto mesi sino a sette anni) caratterizzato dall'evoluzione del linguaggio e dal pensiero simbolico: in questa fase il bambino è in grado di manipolare, oltre che azioni manifeste, anche immagini e simboli. Secondo Piaget, durante questo stadio la prospettiva del bambino è egocentrica; c) lo stadio delle operazioni concrete (dai sette ai dodici anni) caratterizzato da regole di raggruppamento (tra cui quelle di classificazione, conservazione e seriazione); d) lo stadio delle operazioni formali (a partire dai dodici anni) caratterizzato dalla possibilità di trascendere il dato empirico e pensare che le cose potrebbero essere diverse da quelle che appaiono o sono: tipica di questa fase è la possibilità di apprendimento delle principali operazioni logiche e la capacità di scoprire incoerenze logiche del tipo "se A o B portano a Z, è falso affermare che se non si verifica $B$ non si verifica nemmeno $Z$ ”. Il processo di assimilazione cognitiva è regolato tanto da meccanismi di apprendimento, quanto dall'ambiente esterno: senza il supporto dell'ambiente non esiste, secondo Piaget, maturazione del bambino. Le teorie piagetiane relative ai processi di apprendimento sono state oggetto di discussione tra gli psicologi e da molti di loro contestate. Per un completa visione della teoria piagetiana, rimandiamo al testo di Mussen P.H., Conger J.J., Kagan J., Child Development and Personality, trad. it. 1981, Lo sviluppo del bambino e la personalità, Zanichelli Editore, Bologna.

${ }^{34}$ Leroi-Gourhan A., 1986, Meccanica vivente, Jaca Book, Milano.
} 
terno delle molteplici ipotesi che sono state elaborate sull'origine della musica. Fu Géza Révész ${ }^{35}$ a formularla per primo, corroborandola di una micro-teoria interna definita da lui stesso "teoria del richiamo." Sebbene sia lontano dall'attribuzione di semanticità al linguaggio musicale, egli ritiene che l'origine della musica, nel processo evolutivo, sia dipesa dalle stesse esigenze che hanno favorito la nascita del linguaggio verbale. Sia il linguaggio verbale sia la musica rispondono alla necessità di stabilire un contatto con l'ambiente circostante, indipendentemente dal fatto che sia abitato o meno da altri esseri umani. Per questi motivi, secondo Révész, l'uomo ha sviluppato il grido di chiamata e, successivamente, il grido di rimando. Queste due "forme sonore primitive" si sarebbero così strutturate all'interno di atti comunicativi veicolati dall'ecosistema in cui si verificavano: in situazioni ambientali e spaziali ravvicinate il grido di chiamata si sarebbe caratterizzato con poca intensità e durata relativamente breve, il contrario in situazioni ambientali più spaziose. Da questa pratica, reiterata nel tempo, è probabile che sia nato il gusto di ascoltarsi, vale a dire il gusto di produrre delle grida non direttamente funzionali alla chiamata o richiamo.

Sebbene questa teoria sia la più verosimile scientificamente e rispettosa delle concezioni darwiniane, è da ritenere che ancor prima delle grida di chiamata e di rimando sia stato necessario per l'Homo imparare ad imitare ciò che ascoltava attorno a sé e, solo successivamente, abbia raffinato queste imitazioni a fini comunicazionali.

L'individuazione di una qualsiasi fonte sonora non è cosa di poco conto, poiché favorisce la cognizione spazio-temporale di se stessi. La possibilità di stimare la provenienza di un suono, la sua durata temporale e le caratteristiche che lo definiscono ${ }^{36}$ permettono all'uomo di formulare una serie di ipotesi d'azione. Ci si può allontanare dalla fonte perché ritenuta pericolosa, oppure "tendere l'orecchio" per capire meglio le sue "intenzioni"; oppure ancora avvicinarci perché è "fonte di piacere". Tutte queste azioni sono, in realtà, delle reazioni, delle risposte comportamentali all'ascolto e sono subordinate ad una serie di operazioni associative che l'individuo deve compiere per ottenere delle informazioni soddisfacenti. In primo luogo, è necessario procedere all'individuazione delle quattro caratteristiche del suono ${ }^{37}$, in seguito è necessario determinare l'associazione di almeno una di queste con eventi sonori della propria esperienza passata. Questa associazione può con-

\footnotetext{
${ }^{35}$ Cfr. Révész Géza, Einführung in die Musikpsychologie, A. Francke Ag. Verlag, Bern, trad. it. 1983, Psicologia della musica, Giunti Barbera, Firenze, pagg. 240-244.

${ }^{36}$ Le macro-caratteristiche distintive del suono, definite anche "qualità", sono: altezza, intensità, timbro e durata. La percezione dell'altezza di un suono ci permette di distinguere fra suoni alti e suoni bassi. L'altezza dipende dalla frequenza (numero di vibrazioni al secondo) del corpo vibrante. Quanto maggiore è la frequenza, tanto più alto è il suono e viceversa. Il limite minimo percepibile dall'uomo varia dalle 16 alle 20 vibrazioni al secondo, mentre quello massimo dalle 20.000 alle 25.000 vibrazioni al secondo. L'intensità è relativa alla "forza" dell'emissione sonora. In termini fisici, dipende dall'ampiezza della vibrazione. Una più o meno ampia vibrazione produce rispettivamente suoni più o meno forti. Il timbro definisce la differenza del colore del suono. È per esempio, il "timbro" a permetterci di distinguere il suono del violino da quello del pianoforte. La durata si riferisce all'organizzazione temporale del suono: le note possono essere più o meno lunghe/ corte nella loro durata e per questo motivo sono notate differentemente nella partitura. Cfr. Károly O., 1965, Introducing Music, Penguin Books Ltd, Harmondsworth, Middlesex, trad. it. 1969, La grammatica della musica. La teoria, le forme e gli strumenti musicali, Piccola Biblioteca Einaudi, Einaudi Editore, Milano.

${ }^{37}$ Le quattro caratteristiche del suono sono: altezza, intensità, durata e timbro. Cfr. a questo proposito il cap. 1.
} 
durre a risultati positivi o negativi. Nel primo caso, colui che ascolta è in grado di collocare le caratteristiche del nuovo suono all'interno del proprio vissuto esperienziale e potrà quindi esserne in qualche modo rassicurato. Nel secondo caso, egli non è in grado di riconoscere il suono, ma può collocarlo nella propria passata esperienza: esso risulterà quindi oscuro e potrà creare allarme. Qualora si ascolti un suono che non si riesce ad identificare, le reazioni sono imprevedibili ed entrano in azione dei "comportamenti immaginativi". Questi ultimi saranno frutto della cultura cui si appartiene o potranno contenere una dose più o meno ampia di soggettività.

Nella peculiarità di quest'ultima risposta risiede una parte importante del potenziale rappresentativo e creativo dell'uomo. Il nucleo originatore dell'immaginario collettivo risiede proprio in questa capacità-potenzialità individuale, che a sua volta rappresenta il frutto di una sintesi di elementi immaginativi culturali e individuali. Lo spazio che si apre, nel corso dell'esistenza, fra la propria memoria e l'immaginario collettivo favorisce lo sviluppo di scelte interpretative personali collegate alla eventuale produzione di gesti significanti. Nello spazio di questa differenza si insinuano le scelte interpretative dei suoni, siano esse logiche o affettive e, in ogni caso, strutturate sotto forma di comportamenti, o meglio di condotte sonore. Il sentimento di identità personale che riempie questo spazio dipende da una molteplicità di fattori e verrà misurandosi con le dimensioni psicobiologiche dell'intera comunità. Il luogo d'incontro privilegiato di queste molteplici soggettività risiede, comunque, nel potenziale di autorappresentazione collettivo e trova la sua più completa espressione in alcune pratiche sociali: fra le molte, una è senza dubbio la produzione sonora e musicale che rappresenta un settore dell'immaginario collettivo.

Per condotta si intende un insieme di atti coordinati e organizzati per il raggiungimento di un fine. Ogni uomo persegue dei fini propri che si devono adeguare in qualche modo ai fini della collettività. Questo processo si esplica nella oggettivazione delle condotte soggettive, le quali si struttureranno, se legittimate, in comportamento di status, cioè in ruoli ${ }^{38}$. Nelle società più tecnologiche della nostra epoca la quota di oggettivazione delle condotte soggettive si è dilatata al massimo grado (e sembra inutile elencarne le ragioni). Si può persino azzardare che sia impensabile una condotta soggettiva. Tra gli uomini delle origini è invece ipotizzabile che il processo di oggettivazione delle condotte si presentasse come necessità funzionale alla sopravvivenza del gruppo stesso.

Le reazioni al suono (del produttore come del fruitore) tendono esse stesse ad organizzarsi in condotte sonore aventi un alto grado di legittimazione culturale. L'uomo ha infatti avvertito la necessità, nel corso dell'evoluzione, di utilizzare canali comunicativi che realizzassero una condotta di sintesi fra i propri sentimenti ed emozioni - aspetti inerenti al nostro sistema cerebrale rettiliano ${ }^{39}$ e limbico - e l'integrazione nel gruppo attraverso la messa in scena sonora di se stesso ${ }^{40}$.

\footnotetext{
${ }^{38}$ Cfr. Morino Abbele F., 1984, Introduzione alla psicologia sociale, Giunti Barbera, Firenze.

${ }^{39}$ Cfr. Chiarelli B., 1997, Evoluzione del cervello e nascita dell'autocoscienza, in Systema Natura. L'uomo, la natura e il pensiero, vol. 4, Edizioni Polistampa, Firenze.

${ }^{40}$ Cfr. Musio G., 1985, La mente culturale. Struttura della cultura e logica della organizzazione della mente, Università di Firenze, pagg. 65-75 e pagg. 79-80.
} 
Il tema del rapporto tra immaginario e creatività è, tuttavia, molto complesso e non può essere ricondotto esclusivamente alla produzione sonora o artistica in genere. Ci interessa qui porre l'accento sul ruolo svolto dal silenzio-ascolto e dall'udito nella formazione, dapprima, di un dialogo sonoro con la natura e, in un secondo momento, di un dialogo sonoro-simbolico. I presupposti biologici della creatività umana possono essere rintracciati facendo riferimento ad alcune possibilità ad essa collegate: la possibilità di accedere alle proprie potenzialità percettive e, in seconda istanza, quella di elaborare le proprie potenzialità espressive. Il flusso immaginativo risulta così una sintesi di aspetti biologici e creativi, che inscena un ponte emotivo fra ogni singolo Io e gli altri. In questo senso la musica può essere considerata un linguaggio poiché rimanda ad un significato emotivo esterno alla sua stessa struttura frequenziale ${ }^{41}$. Katherina Susanne Langer ${ }^{42}$ parla della musica come di una struttura simbolica non consumata. Ritiene che la consumazione, intesa come attribuzione di significato al simbolo, si attui nel vissuto esperienziale del singolo. Questa teoria ha trovato molti oppositori (da Hanslick a Jakobson), specialmente fra coloro che non vedono nessuna possibilità di collegamento fra la musica ed il linguaggio verbale. Quest'ultimo è infatti semantico, mentre la musica è in sé asemantica. Delalande ritiene che ci si dovrebbe chiedere: "Perché la gente fa o ascolta la musica, che cosa cerca in essa, che cosa ci trova?" Posta la questione in questi termini, la domanda sul significato dell'oggetto musicale si trasferisce sul significato di una pratica attiva sia per il produttore sia per il fruitore. La musica diventa, quindi, un fascio di condotte, che comprende il produrla (ossia comporla ed eseguirla) e l'ascoltarla. "La triplice dimensione - di oggetto, oggetto di produzione, oggetto di ricezione - è ciò che conferisce alla musica il suo statuto di oggetto simbolico" 4 .

L'ascolto del suono (che successivamente si può strutturare in musica) o la sua stessa produzione determinano lo sviluppo di una progettualità interpretativa dello stesso materiale sonoro. I processi imitativi - caratteristici dello sviluppo filogenetico e ontogenetico insieme - rappresentano un primo esempio di questa progettualità e sono di fondamentale importanza per l'essere umano. Essi giocano un ruolo decisivo nella determinazione del rapporto fra l'individuo stesso, il gruppo e l'ambiente: in questo senso, l'imitazione rappresenta il primo esempio di risposta emotiva accolta da una struttura comunicativo-linguistica. Ritroviamo questa reazione nella generale disponibilità all'imitazione cantata di ciò che si sta ascoltando. Cantare è, infatti, una condotta musicale che investe altre dimensioni sensoriali: la propriocezione, l'udito, la postura, etc. In questo caso la musica entra a far parte di una dimensione biopsicologica, innescando relazioni costanti con altri comportamenti espressivi ${ }^{45}$, $\mathrm{i}$ quali, strutturati in qualsiasi forma, potranno venir legittimati in parte

\footnotetext{
${ }^{41}$ Il termine linguaggio è stato definito come "l'intero corpo di parole e metodi di combinarle utilizzati da una nazione". Possiede, però, altri significati: "metodo di espressione, che non usa le parole", oppure "maniera o stile di espressione", o ancora "stile di una composizione". Cfr. , 1959, Shorter Oxford Dictionary.

${ }^{42}$ Langer S.K., 1975, Feeling and Form, trad. it. di Lia Formigari, Feltrinelli Editore, Milano.

${ }^{43}$ Delalande F., op. cit. 1993a, Clueb, pag. 245.

${ }^{44}$ Delalande F., 1993a, pag. 249.

${ }^{45}$ Spesso i musicisti ignorano questi aspetti biopsicologici nelle loro composizioni, anche se inconsciamente ne fanno largo utilizzo. Ad esempio, inseriscono frequenze sintoniche con il respiro o il ritmo cardiaco stimolando calma o staticità; altre volte inducono al dialogo (come nelle sonate a quattro mani o nella musica da camera); in altre
} 
o totalmente dal gruppo ${ }^{46}$. Esistono condotte che contengono elementi minacciosi per il gruppo (perché portatrici di un potenziale negativo in grado di provocare la sua stessa disgregazione), mentre altre sono portatrici di un potenziale positivo (particolarmente adatte a favorire il rafforzamento del gruppo) ${ }^{47}$.

Il "sistema" della cultura rivolgerà la propria attenzione ad entrambe queste condotte, cercando di isolare le prime e rafforzare le seconde. La legittimazione delle condotte sonore (oggettivazione delle condotte soggettive) dipenderà quindi dal loro potenziale espressivo-comunicativo e dalla loro capacità di stimolare un alto livello di integrazione socioculturale. In questo modo, alcune condotte sonore assumeranno il ruolo di una vera e propria autorappresentazione del gruppo che le esprime. Il suono che si struttura in musica diventa così una delle espressioni privilegiate nelle quali gli uomini possono riconoscersi e rappresentare le proprie emozioni ed affetti.

Poiché la musica esiste in quanto è eseguita, la notazione musicale raccoglie in sé la memoria delle pratiche sociali dalle quali deriva, rinnovandole ad ogni nuova esecuzione: traduce in simbolo le condotte sonore di chi la produce, di chi la esegue e di chi ne fruisce.

Nella storia della musica occidentale possiamo rintracciare importanti esempi di questa simbolizzazione delle condotte sonore. Basti a questo proposito ricordare qui come J. S. Bach con il suo "Clavicembalo ben temperato" ha notato grammaticalmente e sintatticamente le condotte musicali che si erano venute da tempo stabilizzando nella gestione dei rapporti di frequenza dei suoni, oggettivandole e legittimandole nella cultura occidentale.

\section{Riflessioni sulla notazione musicale}

Nella premessa ci siamo brevemente soffermati sugli importanti risultati degli studi etnomusicologici, perché riteniamo che il contributo fornito da questi studi sia stato - e sia tuttora - essenziale per una ricerca sulle origini e gli sviluppi dell'oggetto-soggetto sonoro sul nostro pianeta.

Tenteremo ora di oltrepassare i problemi classificatori per porci quelli inerenti le cause delle diversità esistenti tra le differenti culture musicali nel mondo.

Nella sua ricerca dei fattori culturali e sociali in grado di far luce sulle cause di così ampie differenze, John A. Sloboda traccia un'ipotesi che ci sembra particolarmente interessante: egli ipotizza che le forme della musica occidentale, e la natura delle abilità cognitive che sono alla loro base, siano strettamente legate all'esistenza di un sistema sviluppa-

composizioni stimolano depressione, ossessione, maniacalità, come nel caso di “clusters" compositivi poco razionalizzabili. Per questi motivi, le proprietà strutturali dei brani musicali possono innescare negli ascoltatori evidenti miglioramenti della percezione telemetrica o rivitalizzare l'immaginario personale attraverso una sensazione di creatività.

${ }^{46}$ Questa convinzione antropoculturale è anche in F. Delalande, il quale la applica al contesto musicale: "fare musica è prima di tutto un atto motorio che, per effetto della sua funzione sociale di scambio, si arricchisce di una dimensione simbolica e si conforma a delle regole.” Delalande F., op. cit., 1993b, Clueb, pag. 53.

${ }^{47}$ Un esempio di condotta sonora minacciosa potrebbe essere quello in cui un individuo imita (in modo burlesco e grottesco) il canto di un artista, particolarmente amato da coloro che partecipano alla festa. Qualora il suo atteggiamento diventi una vera e propria condotta (ossia reiterato per tutta la durata della festa), il suo comportamento è giudicato minaccioso ed il gruppo cercherà di porre fine alla minaccia. 
to di notazione musicale. Secondo questa tesi, la notazione favorirebbe l'evoluzione di precise forme musicali, legate ad altrettante pratiche musicali che definirebbero le prime. 亡̀ noto tuttavia che non tutte le culture del nostro pianeta hanno utilizzato dei mezzi di notazione per le loro transazioni e comunicazioni interpersonali. Esistono, o per lo meno sono esistite sino a non molto tempo fa, culture sostanzialmente non alfabetizzate (o orali, o astoriche).

Tra gli elementi che distinguono le culture alfabetizzate da quelle orali, Sloboda ne individua quattro di grande significato. Secondo la sua analisi, l'esistenza di una notazione scritta:

a) consente di richiamare elemento per elemento, per esteso, del materiale complesso dotato di significato;

b) consente una proliferazione e una diffusione del materiale, in misura tale da oltrepassare la capacità che un singolo individuo può avere di conoscerlo nella sua interezza;

c) facilita la separazione dei contenuti di un'espressione dal contesto e rende più facile trattarla come un "qualche cosa di per sé";

d) seleziona alcuni aspetti del suono che vanno conservati, e in questo incorpora le teorie del momento, ma tende anche a delimitare i futuri sviluppi della comunicazione in determinate direzioni ${ }^{48}$.

È interessante soffermarsi subito a riflettere sui vantaggi e gli svantaggi dell'alfabetizzazione nei confronti della cultura orale. I vantaggi appaiono evidenti e possiamo limitarci a elencarli sinteticamente:

- una maggiore disponibilità e permanenza delle conoscenze, e quindi

- lo sviluppo di strutture sociali e conoscitive complesse e geograficamente diffuse.

Meno evidenti appaiono gli svantaggi: e tuttavia questi svantaggi esistono, nonostante la diffusa opinione che l'alfabetizzazione dia all'uomo nuove risorse, senza nulla togliergli. Vediamo dunque di evidenziarne qualcuno.

48 È opportuno tener presente che la distinzione tra cultura alfabetizzata e cultura orale è tutt'altro che nettamente segnata. "Le culture contemporanee - fa notare Sloboda - possono essere collocate lungo un continuum, da quelle in cui non vi è alcuna forma di alfabetizzazione, a quelle in cui la lingua scritta pervade tutti gli aspetti delle attività sociali. Vi sono molte culture semialfabetizzate, in cui la scrittura è presente per classi sociali ridotte, o per situazioni limitate, e i cui effetti penetrano sino ai membri che si servono solo di mezzi orali. Anche nelle culture prevalentemente alfabetizzate, come la nostra, possono esserci individui illetterati, che però si dedicano ad attività che hanno alla base, direttamente o indirettamente, la lingua scritta utilizzata dagli altri. (...) Alcune persone alfabetizzate musicalmente si dedicano ad aree di attività musicale, come il jazz o la musica folk, che sostanzialmente non richiedono una notazione scritta. A rendere oggi più complicato qualunque tentativo di demarcazione in questo settore, è anche l'esistenza di mezzi che svolgono molte delle funzioni della scrittura (oltre ad avere, rispetto a quella, proprietà e possibilità aggiuntive) e che pure possono essere utilizzati anche da persone non alfabetizzate: basti pensare, a questo proposito, alle registrazioni elettrofoniche e fotografiche." Cfr. Sloboda J. A., op. cit. 
1) In una società completamente orale, le conoscenze vengono conservate attraverso le consuetudini e i riti che la tengono unita: la limitazione delle conoscenze relative a ciò che è importante per quella società (e che merita quindi di essere incluso nella sua tradizione orale), garantisce che ogni individuo sappia le cose di cui necessita. In una cultura che possiede la scrittura, le conoscenze immagazzinate sono invece così numerose da rischiare di diventare non riconoscibili e non dominabili. È infatti certo che una persona potrà entrare in contatto solamente con una loro piccolissima frazione e che, per utilizzare le conoscenze che gli servono, sarà costretta a dipendere da altri "esperti". La quantità di conoscenze "inutili" (nei confronti delle necessità di un particolare individuo o gruppo) si amplia notevolmente. Questo può significare, nel migliore dei casi, che una persona perderà una gran quantità di tempo e di energie prima di scoprire delle conoscenze utili, nel peggiore, potrà essere gravemente fuorviata da conoscenze inidonee alla sua situazione.

2) La possibilità che la notazione scritta ci offre di distanziarci dalle parole, nostre ed altrui, di analizzarle, sezionarle e, di conseguenza anche eventualmente correggerle, arricchirle, potenziarle (possibilità che rappresenta certamente uno dei grandi vantaggi della cultura alfabetizzata), non è tuttavia esente da svantaggi e da rischi. È certo, infatti, che questo mezzo “incoraggia anche la formazione di un'immagine di noi stessi come separata dalle nostre parole e dalle nostre azioni. Vediamo noi stessi come se osservassimo il mondo, e agissimo su di esso, ma non come se ne facessimo parte" ${ }^{\prime 4}$.

3) La nostra scrittura alfabetica conserva solo le informazioni fonetiche: non è in grado di preservare informazioni tuttavia significative del linguaggio ${ }^{50}$ (come l'intonazione, il ritmo, i gesti, etc.). Questo può portare ad un impoverimento della comunicazione e della sua evoluzione.

Abbiamo così individuato alcuni aspetti generali relativi all'alfabetizzazione: vediamo se considerazioni analoghe possono essere fatte per la musica.

Per trattare questo argomento sembrano opportune alcune precisazioni sulla notazione musicale. Riflettiamo, prima di tutto, sulle varie dimensioni del suono in generale. Le caratteristiche tradizionalmente riconosciute come distintive di un suono (durata, altezza, timbro, intensità: tutte tra loro significativamente condizionate) possono variare con continuità. La musica in generale, e quella vocale in particolare, non è dunque necessariamente costituita di elementi discreti. Tuttavia, il nostro sistema notazionale ha in sé incorporato proprio quel "discreto" al quale parrebbe che la musica non dovesse facilmente assog-

\footnotetext{
${ }^{49}$ Cfr. Sloboda J.A., 1985, op. cit.

${ }^{50}$ Sloboda sta facendo riferimento all'aspetto "prosodico" della fonazione-comunicazione. Il concetto è altresì esaminato da Wallin N.L. nel suo articolo Pensare in termini evolutivi alla musica - una analisi, in Bertirotti A. (a cura ), Systema Naturae. Dai suoni della natura alla musica dell'uomo. Vol. 5 (in stampa).
} 
gettarsi. Affinché un sistema di notazione musicale possa funzionare sembra, infatti, che gli elementi che lo costituiscono (e attraverso la cui combinazione si può costruire tutta la musica) debbano avere almeno tre caratteristiche: devono essere finiti, discreti e in numero ridotto. Questa apparente contraddizione non dovrebbe sorprenderci. Noi sappiamo che nelle culture orali di oggi, pur nella grande libertà praticata nell'uso di abbellimenti microtonali e microritmici, è presente il discreto. Ma soprattutto sappiamo che, dalla preistoria, gli strumenti musicali hanno posseduto, almeno nell'altezza, quelle caratteristiche di discretezza che sono poi state codificate dalla notazione convenzionale.

D'altra parte, la prima educazione musicale scolastica si basa sul canto "intonato" e "a tempo", che equivale per gli studenti ad evitare scivolamenti di altezza, e grandi deviazioni dalle suddivisioni della durata. "Nell'esecuzione, la musica è così come quella scritta, sotto alcuni aspetti cruciali. Le spiegazioni dei concetti di altezza e di durata nella teoria musicale convenzionale implicano il concetto di discreto con l'elaborazione delle nozioni di scala ${ }^{51}$, metro, e così via. Così la teoria, la notazione e la pratica esecutiva costituiscono un sistema chiuso, in cui tutti gli elementi si sostengono a vicenda, ed entro cui la musica può essere scritta, descritta, trasmessa e capita" ${ }^{52}$.

È importante a questo punto sottolineare come il concetto unico di libertà e capacità interpretativa mantenga, pur nella selettività della notazione musicale, il suo pieno significato e valore. La notazione fissa un livello di dettaglio tale per cui si rende possibile stabilire che una certa esecuzione riproduce quel brano e non altri: ma un'esecuzione, culturalmente soddisfacente, di un brano significativo richiede certo molto di più di una semplice capacità di lettura della musica. Richiede, infatti, un buon livello di condivisione emotiva che si esplica in una precisa condotta.

Vediamo ora quali sono le possibili conseguenze e influenze che la notazione esercita sulla musica e sui suoi sviluppi. Il primo problema sul quale ci sembra importante ragionare è quello del mantenimento delle conoscenze nel tempo, ovvero del rapporto esistente tra cultura e memoria. È stato messo in evidenza come l'esistenza di una notazione scritta consenta la riproduzione esatta, elemento per elemento, di un oggetto verbale anche complesso: è chiaro che questa caratteristica si estende alla notazione di oggetti sonori. Nelle culture orali, la trasmissione della musica da un individuo all'altro è soggetta evidentemente a variazioni nel tempo, e non esiste peraltro, all'interno della cultura stessa, la possibilità di controllare se una certa esecuzione riproduce esattamente la stessa musica di prima: solo il generale consenso (configurato in condotta) stabilirà se accettarla oppure no come tale. Ma quel che ci sembra particolarmente interessante è che niente ci assicura che in queste culture si desideri rievocare un dato oggetto musicale sempre allo stesso modo: sta di fatto che la musica viene ricreata da capo ad ogni nuova esecuzione. Quel che gli studi fatti ci hanno rivelato è che "l'apprendimento di determinati brani è reale, nei detta-

\footnotetext{
${ }^{51}$ Una scala musicale può essere definita come il modo attraverso cui l'uomo realizza e nota dei suoni matematicamente e culturalmente discreti. È bene chiarire i termini che definiscono il concetto di scala, soprattutto perché senza di essi nessuna cultura avrebbe potuto produrre "musica notata". La scala è formata da una successione di intervalli; prevede l'utilizzo di un preciso numero di altezze; non vi sono all'interno di essa delle funzioni sonore privilegiate, le quali sono caratteristiche del "modo".

${ }^{52}$ Cfr. Sloboda J.A., op. cit., pag. 382.
} 
gli; ma quel che viene appreso è un'astrazione di livello relativamente elevato da una sequenza di esecuzioni che differiscono nei particolari anche in misura notevole" ${ }^{53}$. In un contesto orale, il musicista ha dunque una struttura già memorizzata in base alla quale è in grado di generare, nelle diverse occasioni, sequenze diverse ma strutturalmente legate. In un contesto alfabetizzato, una capacità di questo tipo viene etichettata come improvvisaz̧ione.

L'esistenza di una notazione esercita anche un significativo influsso sulle forme della musica in una determinata cultura. La notazione rende infatti possibile la costruzione di forme complesse e di lunga durata, programmate secondo una struttura rigorosa che consente l'espansione delle relazioni per centinaia di note. Poiché il compositore ha la possibilità di registrare, momento per momento e in tutti i suoi particolari, l'oggetto sonoro che si viene sviluppando nella propria mente, egli può costruire un brano riferendosi via via, anche ripetutamente, agli eventi annotati-notati precedentemente: la musica così prodotta può, come ben sappiamo, presentare caratteri di notevole complessità architettonica (la forma sonata e la fuga sono due chiari esempi di questa complessità).

In un contesto prettamente orale, le associazioni tematiche appaiono necessariamente più libere, anche se possono musicalmente mantenere efficacia, e si nota una preponderanza di "strutture concatenate", dove un elemento breve può venir ripetuto molte volte con variazioni. La complessità, che è pur presente anche nella musica orale, tende qui a porsi dentro le singole parti, piuttosto che come relazione tra le parti. La struttura del tema con variazioni sembra comunque implicare caratteristiche fondamentali della musica orale, di qualsiasi cultura.

Se proviamo a riflettere sui diversi atteggiamenti con i quali si può ascoltare un brano musicale, ci accorgeremo che è possibile individuarne almeno due: si può cercare di "capire" il brano attraverso il suo contenuto, oppure attraverso il contesto nel quale è stato concepito o eseguito ${ }^{54}$. È chiaro che queste due condotte sono complementari e si presentano entrambe in ogni situazione; tuttavia "è ragionevole che la notazione faccia pendere l'ago della bilancia in direzione del contenuto, tendendo a svalutare il contesto" "55. Questa propensione è sicuramente una conseguenza di quella possibilità di distanziarci dallo scritto, nostro o altrui, di cui ci siamo occupati a proposito dell'alfabetizzazione in genere. Inoltre, la notazione musicale scritta (così come la registrazione dei suoni) consente un esame dei contenuti di un brano, considerato come una "cosa in sé", separandolo dal contesto in cui è stato concepito ed eseguito. Questo distacco non può avvenire nel caso della musica orale, dove il contesto fornisce in qualche misura anche il suo significato.

È opportuno, a questo proposito, ribadire che la musica, avendo a che fare con la fisicità del suono, si esprime sempre attraverso la sua esecuzione e si struttura come un processo acustico assai complesso. Evidenziamo qui di seguito alcuni aspetti di questo divenire sonoro e poniamolo in relazione con le corrispondenti condotte sonore.

\footnotetext{
${ }^{53}$ Cfr. Sloboda J.A., ibidem, pg. 376.

${ }^{54} \mathrm{Al}$ di là degli aspetti strettamente musicali, occorre notare che la relazione circolare suono-uomo-suono (anche nei momenti di produzione e fruizione del suono stesso) è sempre biologicamente e culturalmente connotata.

${ }^{55}$ Cfr. Sloboda J.A., ibidem, pag. 379.
} 
1) La notazione musicale è costituita da simboli che si materializzano attraverso una successione di suoni, i quali, provocati dall'azione dell'esecutore, si trasformano in stimolazioni uditive per il fruitore. In questo senso, la musica prevede un complesso sistema di attività fisiche che contribuiscono a definirla. Chi la esegue fa vibrare delle corde o percuote un tamburo, ed attiva in questo modo un alto livello di consapevolezza e di controllo sensomotorio delle proprie azioni. Chi la ascolta seleziona gli stimoli emotivamente più significativi per lui. In questo senso, tutte queste azioni sono delle condotte sonore che si dispiegano nello spazio e nel tempo.

2) L'esecuzione si colloca nello spazio, sia in senso fenomenico sia in senso oggettivo. I suoni che si percepiscono sono fenomenicamente distanti o vicini a noi, cioè collocati in un luogo spaziale diverso rispetto al nostro luogo di ricezione. Essi sono anche oggettivamente collocati nello spazio, poiché le onde sonore che li costituiscono provengono da un preciso luogo e comprendono una determinata porzione di spazio. La collocazione spaziale del suono non è una proprietà dell'oggetto sonoro in sé, ma una proprietà dei diversi aspetti sonori condivisi sotto forma di condotte da tutti i partecipanti. In altri termini, le nostre sensazioni uditive non risiedono nel suono, ma sono frutto di una nostra precisa condotta verso il suono stesso. Ad ogni suono o formazione di più suoni appartengono aspetti uditivi fisici (durata, altezza, intensità e timbro) e aspetti uditivi psichici (mutamenti dell'attenzione, reazioni emotive) peculiari ad ogni singolo ascoltatore ed esecutore. Nelle preferenze accordate verso gli uni o gli altri si realizzano delle condotte sonore uniche ed irripetibili.

3) Ogni esecuzione (processo) si sviluppa nel tempo e vi si colloca inequivocabilmente. Inizia in un preciso momento, dura un certo periodo di tempo e termina in un altro preciso momento. Non conserva il dono della ripetizione: si possono avere più esecuzioni ma saranno inevitabilmente tutte diverse fra loro ${ }^{56}$. È la forza creativa dell'interpretazione che rinnova le diverse esecuzioni e grazie all'azione combinata degli esecutori e dei fruitori si avranno sempre nuove condotte sonore. Si tratta di un vero e proprio dinamismo creativo in cui la notazione svolge il compito di ricordare a tutti gli attori sonori la pratica socio-musicale che l'ha originata.

L'assimilazione del concetto di musica, intesa come processo acustico scatenante condotte sono$r e$, a quello di pratica socio-musicale pone quindi sullo stesso binario comunicativo esecutori e fruitori e ci permette di attribuire alla notazione musicale quella funzione simbolica di cui abbiamo parlato nella premessa. La musica è in questo senso un insieme di condotte sonore che le conferiscono un alto grado di efficacia simbolica, a sua volta estremamente funzionale ad un tipo di comunicazione che non utilizzi le parole.

Ci rimane da fare un'ultima considerazione che riteniamo non possa essere trascurata. Come avviene per il linguaggio, la notazione musicale seleziona alcuni aspetti del suono che vanno conservati, scartandone altri. Questa selezione condiziona inevitabilmente lo sviluppo della teoria e della pratica musicale e può nel tempo esercitare influenze pesanti

\footnotetext{
${ }^{56}$ È ovvio che non ci si sta riferendo alle esecuzioni di "riproduttori elettronici di suono", ma a quelle dal vivo.
} 
sulla stessa natura della musica. Dalla breve analisi appena condotta su alcuni fatti collegati alla notazione musicale, abbiamo potuto riscontrare come questa sicuramente sia stata alla base di positivi sviluppi. L'evoluzione della musica occidentale negli ultimi cinquantasessant'anni induce a pensare che qualche cosa di negativo fosse stato percepito dai compositori se molti di essi (sulla strada tracciata da alcuni grandi innovatori) hanno, prima, radicalmente rivoluzionato le regole che per lunga tradizione costringevano ad un certo uso della notazione musicale e, poi, oltrepassato del tutto la notazione per esplorare le diverse possibilità creative offerte $\mathrm{da}$ una ricerca diretta e sperimentale di sonorità. Pensiamo, tra l'altro, alla musica "concreta" oppure a quelle opere che utilizzano nuovi mezzi per la produzione dei suoni o, ancora, che adoperano in modo traumatico gli strumenti tradizionali, violando i loro "confini naturali" al fine di ottenere sonorità insolite.

T. Wishart sostiene, a questo proposito, che lo "sterile binario morto" su cui è finita la musica dodecafonica sia dovuto ad una costrizione dell'immaginazione musicale nelle categorie implicite nel sistema di notazione ${ }^{57}$.

Ci sembra indubitabile che proprio la possibilità di superare la notazione, componendo direttamente in suoni con l'aiuto di dispositivi elettronici, abbia consentito ai musicisti di avviarsi verso quella "liberazione dai formalismi" alla quale Wishart fa riferimento: non solo perché non si vede come un qualsiasi sistema di notazione possa affrontare attendibilmente un passaggio continuo tra le altezze, ma anche perché il sistema fisso a dodici note è stato incluso nella tecnologia strumentale abituale (non si possono suonare toni di altezza intermedia con gli strumenti ad accordatura fissa).

\section{Conclusioni}

Nel confrontare i vantaggi e gli svantaggi della notazione musicale scritta, si è osservato che questa presenta, nei confronti della trasmissione orale, tutti i vantaggi che possono derivare dall'acquisizione di una memoria completa e dettagliata (e richiamabile in qualsiasi momento) degli oggetti musicali. Si è anche rilevato come, nello stesso tempo, la notazione può in qualche modo limitare (e di fatto ha limitato) la libertà di evoluzione della musica stessa. Ebbene, la "memoria" del computer ha tutte le caratteristiche di libertà della memoria nelle culture orali, con l'aggiunta della possibilità di documentazione altrettanto dettagliata, e certamente più completa, di quella fornita dalla notazione. Ma non basta: il contesto, che sembrava trascurato nella cultura alfabetizzata - con un comprensibile conseguente impoverimento - può essere totalmente conservato all'interno della realtà virtuale nella quale i moderni computers possono farci navigare. Questo non comporta neces-

\footnotetext{
57 "Nella sua ricerca costante di nuovi modi di espressione - scrive Wishart - la tradizione della musica classica occidentale fu (...) vincolata dal suo concentrarsi strettamente sui rapporti validi in un insieme limitato di altezze che potevano così essere notate, portando al limite il campo dei rapporti armonici scrivibili in questo sistema. Il passo finale fu la tecnica seriale, su dodici toni, integrale, che più che costituire una liberazione da questo ristretto sistema tonale, deve essere considerata in prospettiva storica la capitolazione finale ai dettati permutazionali finitistici di un sistema analitico e razionalizzato di notazione, e la via per una gran quantità di sterili formalismi razionali (...). Utilizzi più sottili dell'altezza, come le inflessioni dei glissati, che possono variare per infinità di velocità, intervalli e curve possibili... non sono riconducibili a questo approccio finitistico." Wishart T., Musical writing, musical speaking, in Shepherd J., Virden P., Vulliamy A., Wishart G. e T., 1977, Whose music? A sociology of musical language, London, Latimer.
} 
sariamente un congelamento dello stile esecutivo: sembra infatti possibile rintracciare, attraverso una rappresentazione virtuale delle condotte, il processo storico che le ha generate e le diverse condotte sonore di fronte allo stesso prodotto musicale notato.

Aggiungiamo a tutto questo la possibilità di suonare senza utilizzare i tradizionali strumenti musicali, producendo quindi sonorità completamente libere dalle costrizioni che questi strumenti hanno incorporato. Si comprende, così, come questo insieme di qualità apra per la musica possibilità sino ad oggi assolutamente impensate.

Tutto questo renderà inutili gli spartiti e la stessa notazione? E ancora: renderà inutili gli “strumenti musicali”? Domande come questa si affiancano ad un'altra domanda ricorrente (collegata all'esistenza del computer e della televisione): scompariranno i libri?

Naturalmente è impossibile leggere nel futuro, e tanto meno in un periodo di trasformazioni così freneticamente veloci. Il nostro istinto e il nostro ragionamento ci portano a ritenere (o almeno ad auspicare) che niente debba scomparire; in particolare, debba invece sempre più diffondersi e trovare utilizzazione e consensi tutto ciò che favorisca la serena riflessione, la possibilità di sviluppare con agio l'intelligenza e la sensibilità di cui siamo dotati: libri, spartiti, strumenti musicali (così come cavalletti e pennelli...) sono appunto strumenti che riteniamo non sostituibili per il perseguimento di questi scopi.

Scrive Delalande: "Non è detto che il periodo di empirismo e sperimentazione in musica non sarà finito tra qualche decennio e che la scrittura al terminale del computer non si riallaccerà alla tradizione della scrittura a tavolino, appena un po' modificata..." 58 . Saggia considerazione, venendo per di più da uno studioso che sembra talvolta tentato di identificare il "musicista" con il "musicista concreto"! Ma a noi sembra che il processo evolutivo (ed anche, sotto certi aspetti, degenerativo) abbia ormai assunto un ritmo talmente accelerato che i tempi non possano più essere attesi con tanta calma.

Decenni? Chissà! Forse è vero che "i musicisti contemporanei hanno già cominciato ad accorgersi (...) che uno sviluppo scatenato della musica elettronica finisce col condurre alla sterilità e all'aridità" 59. Forse quel "riallacciamento" al quale accenna Delalande è già cominciato, forse quel legame non si è mai rotto... Ci sembra tuttavia che mai come oggi, di fronte a tanta possibilità di scelta, sia altrettanto necessario che lo studioso, l'artista, lo scienziato, l'uomo comune volgano il loro sguardo, ben aperto, al futuro e al passato, e si pongano in condizione di riflettere serenamente sugli effetti, sia immediati che a lungo termine, delle loro azioni e delle loro scelte.

\footnotetext{
${ }^{58}$ Delalande F., 1993a, op. cit. Clueb, pag. 153.

${ }^{59}$ Cfr. Sloboda J.A., op. cit., pag. 409. Alle parole riportate sopra, Sloboda aggiunge: "Gli strumenti elettronici devono sempre essere vincolati dai parametri di far musica propri dell'uomo, arricchendoli ed ampliandoli, e non spostandoli verso direzioni arbitrarie."
} 



\section{Musica e linguaggio: una riflessione sui risultati relativi a questo settore di ricerca recentemente pubblicati dalla New York Academy of Sciences}

\section{Introduzione}

I problemi concernenti lo sviluppo e l'apprendimento di capacità cognitive generali e specifiche sono stati e sono tuttora oggetto di ricerca e discussione all'interno di molte discipline. L'esame delle esperienze comuni a tutti gli uomini (per esempio, l'esperienza del mondo fisico e delle sue proprietà) permette di individuare alcuni aspetti precoci dell'acquisizione di abilità ${ }^{0}:$ l'abilità di manipolare oggetti viene generalmente acquisita nel primo anno di vita, mentre occorre attendere il terzo anno per l'acquisizione della competenza linguistica nella lingua naturale.

Alcuni studiosi ritengono che il tipo di apprendimento specifico di determinate età dello sviluppo sia parte di una più generale capacità cognitiva tipica di quell'età. Lo sviluppo cognitivo viene considerato come sequenza ordinata di acquisizioni di nuove abilità e strutture cognitive generali; l'ordine in cui avviene il passaggio dall'una all'altra fase è universale, ed ogni fase è caratterizzata da un processo relativamente rapido di passaggio ad abilità superiori. Questo accade perché l'Homo sapiens applica inconsapevolmente le nuove abilità acquisite al modello più generale delle abilità tipiche di quella fase. Il passaggio avviene quindi attraverso meccanismi di assimilazione ed accomodamento costanti, frutto dell'acquisizione di nuove abilità ${ }^{61}$.

Una diversa teoria ci proviene in particolare dalle ricerche della linguistica chomskya$\mathrm{na}^{62}$. Secondo la teoria della Grammatica Generativa (GG), l'organismo umano è predisposto biologicamente per eccellere in poche specifiche abilità cognitive: una di queste è il linguaggio verbale. Per l'acquisizione di queste abilità esisterebbero particolari meccanismi,

\footnotetext{
${ }^{60}$ Cfr. Sloboda J.A., 1985, The Musical Mind. The Cognitive Psychology of Music, Oxford University Press, trad. it. La mente musicale. Psicologia cognitiva della musica, Il Mulino, Bologna.

${ }^{61}$ Cfr. Piaget J., 1945, La formation du Symbole chez l'enfant, Delachaux et Niestlé, Neuchâtel, trad. it. 1972, La formazione del simbolo nel bambino, La Nuova Italia Editrice, Firenze. La funzione dell'assimilazione è di modificare (integrare) l'alimento (empiricamente "il cibo per il pensiero") incorporato per mezzo dell'interazione con l'ambiente e di conservare la struttura degli schemi operativi. Nella funzione dell'assimilazione, il bambino ristruttura $i$ dati rilevanti dell'ambiente, in modo che esso diventi coerente con i suoi schemi esistenti, cioè, in modo che i dati si adattino a quello che il bambino già conosce. L'assimilazione è la funzione che assicura un significato all'esperienza. La funzione dell'accomodamento è di modificare (differenziare) ed elaborare gli schemi del bambino, in modo da farli diventare coerenti con le caratteristiche dell'ambiente esterno. L'accomodamento dunque integra negli schemi esistenti le nuove informazioni ricevute dall'ambiente, in modo da modificarli senza distruggerli. Lo sviluppo locale a breve termine è un processo attraverso il quale l'assimilazione e l'accomodamento sono messi in equilibrio; un disequilibrio locale si verifica quando l'assimilazione (o accomodamento) diviene la caratteristica dominante dell'azione del bambino. Cfr. Langer,J., 1969, Theories of Development, Holt Rinehart and Winston Inc., New York, trad. it. 1973, Teorie dello sviluppo mentale, Giunti Barbera, Firenze.

${ }^{62}$ Cfr. Chomsky N., 1970, Syntactic structures, The Hague, Mouton, trad. it. Le strutture della sintassi, Laterza, Bari; 1969-1970, Aspects of the Theory of syntax, Cambridge, Mass., MIT Press, trad. it. Aspetti di una teoria della sintassi, Boringhieri, Torino; Language and mind, New York, Harcourt Brace Jovanovitch.
} 
che non fanno corpo unico con capacità cognitive più generali, ma si limiterebbero (almeno all'inizio) alla specifica abilità in questione.

Le due concezioni teoriche alle quali abbiamo accennato non sembra debbano necessariamente essere incompatibili tra loro. Secondo Gardner e Wolf ${ }^{63}$, anzi, è necessario ricorrere ad entrambe se si vuol spiegare in maniera soddisfacente il decorso dello sviluppo cognitivo generale. Secondo questi autori, lo sviluppo umano sarebbe caratterizzato da correnti o strutture distinte di acquisizioni di abilità specifiche (aventi con ogni probabilità dei meccanismi biologici alla base) e da onde di simbolizzazione o elementi della struttura comuni, grazie alle quali ogni nuova acquisizione tipica di una corrente si "travasa" in correnti apparentemente prive di rapporti.

"Sino a pochissimo tempo fa - scrive John Sloboda - nessuno aveva studiato la possibilità di modificare delle abilità cognitive generali con un'attività educativa indipendente da abilità specifiche. Sembra piuttosto che il "travaso" tra abilità sia una propensione umana spontanea. È possibile che una caratteristica definente del pensiero umano consista nell'esistenza di un qualche mezzo mentale, abbastanza astratto da consentire il passaggio di abilità generali tra due diverse abilità specifiche" ${ }^{34}$.

Queste schematiche indicazioni generali sono forse sufficienti a chiarire che l'impostazione della ricerca, nel settore specifico dello sviluppo musicale e linguistico, appare comunque diversa, a seconda che ci si ponga nell'una o nell'altra delle due concezioni teoriche sopra esposte. Nella prima ipotesi infatti ci si dovrà disporre a ricercare delle invarianti, ad esempio dello sviluppo musicale, probabilmente legate a dei cambiamenti generali che si verificano contemporaneamente in altri domini cognitivi ${ }^{65}$. L'assimilazione di abilità musicali dovrebbe infatti andare di pari passo con conquiste generali tipiche di quella fase cognitiva ${ }^{66}$. Le sequenze di acquisizioni musicali, in base alle quali si vengono sviluppando le capacità musicali, non determinerebbero quindi necessariamente acquisizioni di abilità musicali, ma piuttosto spiegherebbero i tipi di attività musicale riscontrabili nelle diverse fasi dello sviluppo generale. Nella seconda ipotesi, si dovranno ricercare i possibili aspetti più precoci dello sviluppo musicale, che indicano delle capacità specifiche per la musica, non rilevabili in altri domini cognitivi.

\footnotetext{
${ }^{63}$ Gardner H., Wolf D., 1995, The Waves and Streams of Symbolization, in Acquisition of Symbolic Skills, (a cura di) Rogers D. e Sloboda J.A., New York, Plenum.

${ }^{64}$ Sloboda J., 1985, Musical Mind, op. cit.

${ }^{65}$ Nell'ottica piagetiana il concetto di sviluppo è fondamentale per comprendere i meccanismi evolutivi del pensiero. Infatti, l'autore assimila questo concetto a quello di interazione. "La struttura di ogni stadio delimita il campo delle azioni che l'individuo può compiere ad un dato stadio. Quindi determina la specie di interazione che l'individuo può avere con l'ambiente e il tipo di esperienza che incontrerà. Le sue esperienze hanno ripercussioni sulla struttura presente e alla fine (...) la struttura presente viene qualitativamente alterata, fino a diventare la struttura dello stadio successivo. È in questo senso che possiamo interpretare la teoria organismica della causalità materiale dello sviluppo: l'organizzazione degli stadi precedenti implica ma non contiene quella degli stadi successivi." Cfr. Langer J., op. cit., pag. 140.

${ }^{66}$ È infatti nostra convinzione che uno degli aspetti universali della musica risieda appunto nella capacità che ogni individuo possiede di essere musicale, ancorché questa capacità si realizzi a diversi livelli di competenze, con diverse modalità e in diversi ambienti socio-culturali.
} 
Questo tipo di studi ha stimolato un crescente interesse anche per "forme linguistiche" diverse dal linguaggio vero e proprio. Settori della ricerca si sono rivolti con particolare attenzione allo studio dei meccanismi percettivi e cognitivi sottesi all'ascolto e all'esecuzione della musica. La ragione di questo diffuso interesse risiede in parte nella convinzione che la musica rappresenti un'occasione di studio speciale per la scoperta dell'organizzazione della mente umana ${ }^{67}$. Si parte dal presupposto che la musica rappresenti un tipo di simbolizazione di intenzioni e obiettivi umani incorporati nella sua stessa struttura comunicativa $^{68}$. In effetti, come lo sviluppo del linguaggio, anche quello della musica è il risultato di una complessa attività neuronale, strutturata ed organizzata tanto da renderla prerogativa umana ${ }^{69}$. Da questi studi inoltre sembra emergere la convinzione che la musica sia associata ad una specifica architettura cerebrale. Fin dalla nascita, ogni individuo sviluppa inconsapevolmente una personale sensibilità al fatto musicale, ma solo una minoranza degli individui segue un successivo training specialistico, ed è proprio la presenza di questo gruppo che permette di evidenziare i fenomeni cerebrali e cognitivi sottesi alla pratica musicale. Eseguire e comprendere musica sono attività collocate all'interno di generiche acquisizioni culturali, ma l'onnipresenza di queste azioni, le loro caratteristiche e il loro substrato neurobiologico suggeriscono di collocarle come naturalmente legate a meccanismi evolutivi, cioè a strutture biologiche precise.

La ricerca sistematica di questi ultimi anni si è quindi focalizzata in diversi settori di indagine:

a) lo studio delle origini della musica dal punto di vista strettamente biologico;

\footnotetext{
${ }^{67}$ La particolarità della musica risiede nella sua stessa struttura interna e, per meglio dire, nel concetto di andamento. Con il termine andamento si suole indicare il tipo di relazioni esistenti in una successione temporale di due o più suoni. L'andamento della musica può essere scritto o non scritto (cioè notato o non-notato) e, nel primo caso, saremo in presenza di un sistema di segni in grado di indicarne le reciproche relazioni. La notazione musicale è strutturata verticalmente ed orizzontalmente (cfr. Fig. 2). Nella pratica esecutiva, questi due elementi non si percepiscono separati e si integrano in un dinamismo temporale in cui i suoni si succedono contemporaneamente e cronologicamente. Questi due assi cartesiani musicali (verticalità e orizzontalità) costituiscono l'architettura della musica. La giustapposizione di due o più suoni in successione cronologica conferisce alla musica un andamento accordale. Mettere una nota contro l'altra (punctum contra punctum, definizione risalente al XIII sec.) è l'arte di combinare una melodia data con una od altre melodie, più o meno autonome. La lettura orizzontale di queste melodie conferisce un carattere polifonico alla musica, mentre una lettura verticale le conferisce un carattere armonico. Più avanti parleremo di questo aspetto quadridimensionale.

${ }^{68}$ Treitler L., 1980, History, criticism and Beethoven's Ninth Symphony, Nineteenth Century Music 3, pagg. 193-210.

${ }^{69}$ Esiste in effetti una serie di "caratteristiche" che fanno pensare alla musica come risultato di un adattamento evolutivo:
}

a) in tutti gli adulti umani sani si riscontra un'elevata capacità musicale rispetto alle specie imparentate.

b) la musica coinvolge delle specifiche tecniche mnemoniche, tali da permettere la riproduzione di melodie tra migliaia di quelle conosciute;

c) l'espressione delle capacità musicali - come vedremo nel corso di questo scritto - coinvolge a livello encefalico una notevole lateralizzazione corticale e localizzazione specialistica;

d) la musica dell'uomo presenta delle analogie con alcuni suoni di altre specie, suggerendo una sorta di evoluzione convergente;

e) la musica, procurando a volte delle forti emozioni, può determinare l'insorgenza di atteggiamenti e stili comportamentali finalizzati alla ricezione o meno dei suoni stessi. 
b) lo studio delle strutture cognitive che evidenziano l'architettura della "mente musicale" in ottica funzionalistica;

c) lo studio delle attivazioni e localizzazioni delle popolazioni di neuroni sottese a tutti i processi musicali.

Lo studio dei processi elaborativi che caratterizzano la musica e il loro confronto con quelli che caratterizzano il linguaggio può quindi rappresentare un notevole passo avanti per la ricerca neuro-cognitiva. In effetti, il materiale della musica (note e suoni) per essere in qualche modo razionalizzato, ossia elaborato, deve superare la musica stessa. In altri termini, per comprendere significati, forme, configurazioni, gestualità e simboli musicali è necessario affidarci a codici meta-musicali e, nei casi più consueti, al linguaggio. Gli elementi della musica vengono quindi elaborati cognitivamente anche grazie all'intervento del linguaggio, il quale completa i significati musicali immanenti la musica stessa con altrettanti significati linguistici, frutto di ideologie e culture. La questione può essere quindi affrontata evidenziando similitudini e differenze fra i due tipi di linguaggio, dapprima in ottica evoluzionistica e, successivamente, in ottica cognitivistica.

\section{Similitudini e differenze fra il linguaggio e la musica: l'approccio evoluzionistico e l'approccio cognitivistico}

La questione circa le origini del linguaggio e della musica rimane a tutt'oggi ancora oscura, anche se si sta assistendo, in questi ultimi anni, ad un rinnovato interesse verso lo studio di questi due tipi di comunicazione in chiave evoluzionistica. Le ricerche nei due settori si avvalgono oggi di nuove tecnologie e metodi di indagine che fanno comunque capo alla tecnica dell'immagine funzionale del cervello. Questa tecnica, che fornisce un'immagine spaziale e temporale delle regioni cerebrali, permette di evidenziare l'attivazione di specifiche aree in relazione a particolari input esterni e collocare tali attivazioni lungo un continuum temporale preciso. Sebbene non permetta di stabilire l'origine filogenetica comune o separata del linguaggio e della musica ${ }^{70}$, questa tecnica agevola tuttavia la scoperta di similitudini e differenze fra i due tipi di sistemi elaborativi ${ }^{71}$.

\footnotetext{
${ }^{70}$ In realtà, non è possibile dimostrare l'esistenza di un comune ed immutabile nucleo originario dal quale si sia sviluppata la musica, in tutte le sue diverse forme.

${ }^{71} \mathrm{La}$ "questione delle origini" ha attraversato la storia del pensiero occidentale. Basti accennare a questo proposito alle diverse concezioni di J.J. Rousseau e C. Darwin. Secondo Rousseau, il linguaggio e la musica condividono lo stesso nucleo filogenetico, anche se il primo, in quanto più funzionale ad un'organizzazione razionale della società umana, ha origine dalla seconda (cfr. Rousseau J. J., 1781/1993, Essai sur l'origine des langues, Flammarion, Paris). Anche Darwin individua una stessa origine, ma avanza l'ipotesi che la musica sia lo sviluppo di segnali vocali sessuali di primati e comunque posteriore al linguaggio. In effetti C. Darwin identifica due tipi di selezione sessuale: la rivalità aggressiva e la scelta del partner. La prima è riscontrabile tra i maschi specialmente e tende a stimolare la produzione di "armi": denti aguzzi, muscoli forti, corna robuste e grandi. La seconda, tipica delle femmine, stimola la produzione di ornamenti, colori, suoni innovativi, etc. ̇̀ interessante notare la posizione assunta a questo proposito da Wallin e al. (cfr. Wallin N.L., Merker B., Brown S., 2000, (eds), The Origins of Music, MIT Press, Cambridge, Mass.) che riprende quasi interamente l'assunto teorico di Darwin. Questi autori ritengono che il linguaggio e la musica svolgano entrambi la funzione base di esprimere le variazioni emozionali individuali: nel linguaggio l'emotività si manifesta attraverso cambiamenti dell'intonazione (aspetto prosodico) e del ritmo (velocità di espressione), mentre in musica l'emotività si manifesta nella velocità, accelerazione e altezza delle sequenze sonore. Tuttavia, in questo modo,
} 
Nella cultura occidentale, la musica si è sviluppata in modo tale da diventare ed essere a tutt'oggi sempre più compartecipata, ma quasi totalmente isolata dal resto delle altre forme espressive. La produzione musicale dispone di un uditorio assai vasto, e sono rare le persone ad essa refrattarie. In altre culture, in cui sopravvive una visione magica dell'esistenza, i collegamenti fra musica, canzone, danza, poesia e rito non sono andati perdu$\mathrm{ti}^{\mathrm{i}}$. Inoltre, l'etnomusicologia ha ipotizzato che la musica sia il prodotto finale di atti volontari, ossia il risultato acustico di azioni fisiche quotidiane, e in effetti in molte lingue africane la stretta relazione fra musica ed azione è espressa anche nel linguaggio che utilizza gli stessi termini per indicare sia aspetti musicali sia coreici. Blacking definisce la musica come "suono organizzato all'interno di modelli socialmente legittimati" 74 . Egli considera, in sostanza, la musica espressione di una capacità cognitiva, quindi anche affettiva, che coinvolge la dimensione corporale dell'uomo e si presenta come il riflesso di modelli sociali strutturati culturalmente ${ }^{74}$. Talvolta la sua analisi sembra semplicistica e al di fuori di una soddisfacente elaborazione scientifica: infatti qualora la musica fosse effettivamente uno specchio dei modelli culturali, basterebbe conoscere i meccanismi cognitivi generali di questi modelli per giungere alla comprensione di quelli musicali. In questo modo, si tratterebbe di una corrispondenza biunivoca fra due categorie di oggetti, musicali e culturali. Ci sembra più convincente considerare il rapporto fra musica e cultura sotto forma di soggetti musicali e culturali, proprio perché i modelli sonori (ossia, ciò che Blacking definisce sequenze musicali di suoni organizzati) non sono meri oggetti musicali, ma frutto di costanti processi cognitivo-individuali e cognitivo-culturali, tipici di un particolare ecosistema.

Sono state date molte definizioni di musica. Secondo un'ottica funzionalistica:

"Music can be defined as those temporally patterned human activities, individual and social, that involve the production and perception of sound and have no evident and immediate efficacy or fixed consensual reference" 75 .

Secondo un punto di vista antropologico (che peraltro contiene in sé l'aspetto funzionalistico), è innanzi tutto meglio parlare di musiche più che di musica e quindi

essi condividono l'opinione di Rousseau, secondo il quale le prime forme linguistiche furono cantate, non parlate; furono espressione di emozioni, amore, odio e furore, e quindi legate all'espressione della passionalità più che della razionalità (cfr. Molino J., 2000, Toward an evolutionary theory of music and language, In Wallin N.L., Merker B., Brown S., 2000, op. cit., pg. 165-176).

${ }^{72}$ Schön A. e D., 1999, Il potere del suono e della musica. Fuga a più voci, Psiche 1-2, pagg. 159-165.

${ }^{73}$ Blacking J., 1973, How Musical is Man?, University of Washington Press, Seattle.

${ }^{74}$ È proprio la dimensione culturale che conferisce alla musica il suo carattere polisemico. Per i Kaluli della Nuova Guinea la musica può svolgere la funzione di favorire il contatto con i defunti o con le anime e quindi acquisisce un significato contingente. In altri casi (vedi l'iniziazione domba dei Venda) la musica può favorire la ristrutturazione di relazioni sociali interrotte.

75 "La musica può essere definita come [l'insieme di] quelle attività umane strutturato temporalmente, individuale e sociale, che coinvolge la percezione e produzione dei suoni e non possiede un evidente ed immediata efficacia né una referenzialità consensuale stabilita [a priori]". T.d.A. Cross I., 2001, Music, Cognition, Culture, and Evolution in The Biological Foundations of Music, Annales of the New York Academy of Sciences, vol. 930, New York, pag. 32. 
"Musics are cultural particularizations of the human capacity to form multiply-intentional representations through integrating information across different functional domains of temporally extended or sequenced human experience and behaviour, generally expressed in sound" ${ }^{76}$.

Quest'ultima definizione mette quindi in luce aspetti fondamentali delle pratiche musicali in chiave evoluzionistica: l'apparizione dell'Homo sapiens è stata contrassegnata dall'emergere di domini cognitivi flessibili adatti all'acquisizione di nuove abilità. La musica si è venuta strutturando nel tempo in modo tale da favorire l'acquisizione e il mantenimento di competenze capaci di produrre un adattamento cognitivo ed esistenziale continuo.

Secondo un'ulteriore prospettiva, quella strutturale, proposta da Arom $^{78}$, esisterebbero due criteri generali e strutturali adatti a definire la musica:

a) il ritmo ${ }^{78}$ e i rapporti di tempo, i quali entrambi definiscono la segmentazione temporale degli eventi sonori;

b) la suddivisione in altezze discrete del continuum sonoro, ossia la formazione delle scale $^{79}$.

Questi criteri sono applicabili anche per la definizione di linguaggio. In effetti, anche il linguaggio è costruito su eventi sonori sequenziali che si dispiegano lungo il corso di un "tempo", con un loro specifico ritmo, segmentazione (fonemi) e prosodia. Più in generale, alla luce di quanto si è detto, si può ragionevolmente sostenere che linguaggio e musica presentano una serie di elementi comuni:

a) entrambi utilizzano dei suoni;

b) si basano su elementi presenti in tutte le culture;

c) sono espressioni specificatamente umane;

d) sono determinati dalla cultura.

Inoltre, entrambi utilizzano elementi semplici (fonemi, parole, note ed accordi) strutturati in un sofisticato sistema di regole, a sua volta organizzato in frasi e periodi musicali, argomenti e soggetti letterari, dal quale prendono origine sintassi e armonia.

${ }^{76}$ "La produzione musicale è una particolarizzazione culturale che si dirige verso forme di rappresentazione multiple ed intenzionali, mediante informazioni integrate, le quali si dispiegano lungo domini funzionali diversificati fra loro. Questi domini sono esperienze umane e comportamenti estesi temporalmente od ordinati in sequenza, generalmente espressi attraverso i suoni.” T.d.A. Cross I., 2001, ibidem, pag. 38.

${ }_{77}$ Arom S., 2000, Prolegomeni to a biomusicology, in Wallin N.L., Merker B., Brown S., 2000, (eds), op. cit., pagg. 27-30.

${ }^{78}$ In effetti "One factor that appears to apply to almost all the world's musics is that there is a level of temporal organization that is regular and periodic, sometimes called the tactus". "Uno degli aspetti che sembra possano caratterizzare tutte le musiche del mondo è la presenza di un livello di organizzazione temporale regolare e periodico, alcune volte definito accento". T.d.A. Cross I., 2001, op. cit., pag. 30.

${ }^{79}$ Treitler L. attribuisce proprio a questa strutturazione interna della musica il significato della musica stessa: "Meaning in music is a function of the engagement of codes or orders by the note-complexes of which the musical events is comprised". Treitler, in Shepard R.N., 1992, The Perceptual organization of colours: an adaptation to regularities of the terrestrial world?, in Barkow J., Cosmides L., Tooby J., The Adapted Mind: Evolutionary Psychology and the Generation of Culture, Oxford University Press, Oxford, pag. 203. 
Per questi motivi, si può legittimamente parlare di una "grammatica della musica" come si parla di "grammatica del linguaggio". Tutto questo trova un importante riscontro nei più recenti studi di neuro-biologia della musica, che hanno messo in evidenza sconcertanti similarità fra i diversi tipi di "reattività cerebrale" di fronte a violazioni sintattiche nella musica come nel linguaggio. Queste indagini sono state stimolate dalle ricerche che negli ultimi trent'anni hanno indagato le ipotetiche congruenze fra il linguaggio e la musica. 亡̀ quindi di queste ipotesi che dobbiamo qui cominciare ad occuparci.

Gli studi di psicologia cognitiva hanno definito il linguaggio come una struttura complessa di specialistiche abilità, che si sviluppano spontaneamente fin dall'infanzia e senza nessun aiuto o istruzione formale, qualitativamente simile in tutti gli individui, permettendo l'acquisizione di informazioni utilizzate nel comportamento quotidiano ${ }^{81}$. Al di là di alcuni limiti legati alla mera produzione sonora, questa definizione potrebbe essere facilmente applicata alla musica. Sandra Trehub ${ }^{81}$ e suoi collaboratori hanno infatti dimostrato che anche i neonati possiedono la capacità di percepire strutture musicali significative e alcune loro caratteristiche affettive. I bambini sono particolarmente sensibili ai mutamenti che si verificano nell'ambiente attorno a loro. Ogni suono nuovo, insolito, improvviso catturerà la loro attenzione. L'attenzione del bambino a questi mutamenti (prodotti da altri o da lui stesso) ha inizio molto presto nella generalità dei casi e si manifesta con reazioni che, nel primo anno di vita, hanno carattere circolare $^{82}$. Se qualche suono esterno interrompe il livello di consuetudine uditiva cui il bambino è abituato, egli può rispondere con atti motori ${ }^{83}$. Alcune ricerche dimostrano che a cinque mesi i bambini sono sensibili a cambiamenti sequenziali musicali. Queste ricerche ${ }^{84}$ si sono basate sulla misurazione del ritmo cardiaco come indicatore della percezione di novità. Più i bambini si abituano ad una determinata sequenza di suoni, più il loro ritmo cardiaco si stabilizza. Quando al bambino si presenta una melodia con un contorno cambiato, il ritmo cardiaco si altera, ma ciò non accade se la stessa melodia viene trasposta. Quindi, in questo periodo, la sensibilità al cambiamento è rivolta solo alla melodia e al suo contorno ma non all'altezza dei suoni ${ }^{85}$. Intorno ai nove mesi, i bambini compiono dei vocalizzi distinti per i diversi tipi di stimo-

\footnotetext{
${ }^{80}$ Inoltre, il linguaggio si sviluppa senza la coscienza della logica sintattica sottostante.

${ }^{81}$ Trehub S.E., Schellenberg G. \& Hill D., 1997, The origins of music perception and cognition: a development perspective, in Deliége I., Sloboda J., 1997, Perception and Cognition of Music, The Psychology Press, Hove, pagg. 103-128.

${ }^{82}$ La circolarità della reazione consiste nella ripetizione da parte del bambino di gesti o suoni che lo consolano o gli danno piacere. Piaget stesso ci dice: "accade che il grido di rabbia del bambino vada a finire in un grido acuto che lo distrae dal suo dolore e che prosegue in una sorta di gorgheggio molto breve."

${ }^{83}$ Delalande F., 1993, Le condotte musicali, Editrice CLUEB, Bologna, pag. 55. Il Delalande sostiene che i gesti che il bambino impara a padroneggiare nel periodo senso-motorio acquistano poi un valore simbolico "giocando su un'ambivalenza molto vicina a quella coltivata dalla musica". Esaminando alcune caratteristiche comuni all'attività senso-motoria del neonato e ai movimenti dell'interprete, egli ritiene di poter individuare, da questa base di corporeità, l'emergere di una semiotica del gesto e del movimento nel musicista come nel bambino.

${ }^{84}$ Chang H., Trehub S.E., 1977, Auditory processing of relational informations by young infants, in "J. Exp. Child Psychol"., 24, pagg. 324-331; Infant's perception of temporal grouping in auditory patterns, in "Child Development", 1977, 48, pagg. $1666-1670$.

${ }^{85}$ Esistono tuttavia dei dati a sostegno della tesi secondo cui "il ricordo della linea melodica (l'andare su e giù degli intervalli melodici) è indipendente dal ricordo dell'ampiezza esatta dei singoli intervalli”. Secondo questa tesi
} 
lazioni musicali, denominati da Moog lallazioni cantate. La caratteristica di questo tipo di lallazioni è di non avere alcun riferimento con le altezze dei suoni o con il ritmo.

Di contro, i bambini che hanno più di nove mesi posti di fronte a puri ritmi e rumori dimostrano chiari segni di dispiacere: si girano dall'altra parte, mostrando visi insoddisfatti e talvolta anche segni di paura. I bambini che hanno meno di un anno mostrano di riconoscere brani musicali a loro familiari solo nel caso in cui i genitori cantino molto spesso e siano soliti ripetere le stesse canzoncine. Se questo avviene, Moog ha dimostrato che i bambini sono in grado di associare gesti e movimenti giusti alle melodie che essi riconoscono come familiari.

In conclusione, potremmo dire che il processo di acculturazione musicale nel primo anno di vita ha i seguenti parametri di riconoscimento:

a) i bambini riescono a distinguere suoni casuali da suoni intenzionalmente musicali;

b) riescono a dimostrare questa distinzione attraverso l'aumento dell'attenzione, i movimenti e le lallazioni;

c) le qualità delle produzioni sonore che maggiormente apprezzano sono quelle della voce cantata e dello strumento;

d) verso la fine del primo anno di vita ed immediatamente dopo, i bambini sono in grado di imitare singole note cantate e possono discriminare delle sequenze musicali, se udite più volte di fila ed aventi contorni melodici e ritmici riconoscibili.

Questo breve sunto delle competenze che vanno dalla nascita al primo anno di vita dimostra come il linguaggio e la musica siano atti creativi, che realizzano delle precise intenzioni e che possono essere interpretati solo alla luce di una soddisfacente teoria della mente $^{86}$ : entrambi esigono un certo periodo di tirocinio iniziale che dipende, nella sua strutturazione, dalla "cultura" musicale e linguistica dei quali sono espressione. Inoltre, questo periodo (inculturazione formale) si fonda su di un precedente periodo (inculturazione informale) in cui tutti noi spontaneamente apprendiamo sistemi comunicativi con regole complesse. Il linguaggio e la musica prevedono quindi un buon livello di memorizzazione che agevola l'acquisizione e riproduzione di melodie, parole, poesie e canzoni nuove.

Da quanto esposto emerge chiaramente che la possibilità di evidenziare congruenze e incongruenze fra il linguaggio e la musica dipende, in primo luogo, dal livello di analisi utilizzato nella ricerca. In altri termini, alcune congruenze rilevate ad un certo livello di elaborazione cognitiva potrebbero risultare incongruenze ad un altro livello. Per esempio, mentre la struttura temporale e l'organizzazione ritmica giocano un ruolo determinante in

\footnotetext{
"l'andamento melodico è una astrazione della melodia reale che può essere memorizzata indipendentemente dalle altezze o dalle dimensioni degli intervalli. Ciò è vero sia per la memoria a breve termine, sia per quella a lungo termine". Bowling W.J., 1978, Scale and contour: two components of a theory of memory for melodies, in "Psychological Review", 85, trad. it. Lorenzetti L.M., Antonietti A., 1986, I processi cognitivi nella musica, in Processi cognitivi in musica, (a cura di), Franco Angeli Editore, Milano, pag. 193.

${ }^{86}$ Cfr. Pacherie E., 1960, Philosophie et sciences cognitives, in Encyclopédie Philosophique Universelle, Mattei J.F., Edizioni PUF, Paris.
} 
entrambi, l'aspetto metrico ${ }^{87}$ delle sequenze di suoni costituisce un aspetto specifico del linguaggio musicale che non trova la stessa corrispondenza nell'aspetto prosodico del linguaggio, meno specifico e più variabile. Nello stesso tempo, la segmentazione del continuum sonoro con elementi discreti, che permette la formazione di scale di suoni e che contraddistingue la struttura della musica, trova interessanti riscontri nell'aspetto prosodico del linguaggio parlato o recitato.

Comunque, ammesso che sia plausibile ricercare le differenze come le congruenze, è sempre opportuno avere coscienza della sostanziale alterità della musica rispetto al linguaggio. Infatti, nonostante il numero delle frequenze (altezze) che formano una scala sia pressappoco identico in tutte le culture (sette o poco più), il numero dei fonemi delle diverse lingue varia notevolmente (ad esempio, da undici dei polinesiani a 141 dei boshimani). Inoltre, spesso non è possibile costruire analogie fra elementi musicali ed elementi linguistici. Basti pensare all'esistenza storica ed autonoma del sistema tonale in musica rispetto ad altrettanta autonomia del sistema sintattico dei diversi linguaggi.

Quindi, quando si parla di grammatica della musica si intende in realtà qualcosa di diverso rispetto alla grammatica del linguaggio. Unica cosa certa è che entrambi i codici (musica e linguaggio) sono soggetti a leggi generali di percezione acustica. Ad esempio, il riconoscimento del contorno musicale, ossia della melodia, è equiparabile all'identificazione dell'aspetto prosodico di una frase. In quest'ottica, la grammatica della musica sembra quindi configurarsi come molto più flessibile e imprecisa rispetto a quella linguistica ${ }^{88}$. L'ambiguità è d'altra parte un elemento chiave della grammatica e dell'estetica musicale: grazie a questa flessibilità la musica può essere letta, percepita e goduta secondo diverse priorità. Ad esempio, la scelta di ascoltare un brano secondo il "principio della simultaneità", ossia attraverso una sua lettura verticale comunemente definita rrmonia $^{89}$, è impensabile nel linguaggio, mentre è esperienza comune che sentire più persone che parlano simultaneamente produce nell'ascoltatore solo una spiacevole cacofonia. Abbiamo visto finora che la possibilità di individuare differenze e congruenze fra il linguaggio e la musica dipende, in prima istanza, dal livello che si considera nell'ambito dell'analisi che si conduce. Indipendentemente da ciò è tuttavia palese a tutti noi una fondamentale differenza fra i due tipi di comunicazione: il linguaggio possiede uno spazio referenziale "oggettivo reale", mentre la musica è considerata auto-referenziale ${ }^{90}$. Mentre il linguaggio pone in relazione una serie di suoni (fonemi) con una corrispondente serie di significati culturalmente determinati, le note e gli

\footnotetext{
${ }^{87}$ In realtà, nell'antichità classica si applicava alla poesia, musica e danza un unico sistema metrico. Le sillabe infatti si distinguevano in brevi e lunghe, ossia sulla base della loro durata (esattamente come accade per le note musicali). È anche probabile che l'accentuazione non fosse dinamica ma, in un certo senso, melodica, ossia basata su un aumento dell'altezza vocale, come accade per la lingua cinese.

${ }^{88}$ Aiello R., 1994, Music and language: parallels and contrasts, in Aiello R. \& Sloboda J., Musical perception, Oxford University Press, New York, pagg. 40-63.

${ }^{89}$ "L'armonia è la teoria degli accordi e delle loro possibilità di collegamento con riguardo ai loro valori architettonici, melodici e ritmici e ai loro rapporti di equilibrio". Schöenberg A., 1922, Harmonielehre, Universal Editino, Wien, trad. it, 1991, Manuale di armonia, Il Saggiatore, Mondadori, Milano, pag. 14.

${ }^{90}$ Wallin N.L., Merker B., Brown S., (eds), 2000, The Origins of Music, MIT Press, Cambridge, Mass. L'aspetto auto-referenziale della musica viene spesso definito come "inefficacia comunicativa". In altri termini, la sua "efficacia comunicativa" risiede al di fuori di se stessa, ossia coinvolge l'aspetto edonico della vita individuale.
} 
accordi non si collegano a oggettivi significati extra-musicali. Con questo non si vuole affermare che la musica non possiede un significato simbolico ${ }^{91}$, anzi vi è nella musica qualcosa di simbolico in senso assoluto, ossia qualcosa che ci riporta alle nostre origini più lontane. Nello stesso tempo, la musica è anche costruzione simbolica di aspettative: una nota, un accordo un intero periodo musicale ci indica l'evento musicale successivo, determinando in noi la nascita di precise "aspettative sonore e musicali".

L'aspetto simbolico della musica si collega direttamente ad un problema definitorio più generale non ancora risolto, quello concernente la semanticità della musica. La questione, posta in questi termini, risulta sterile, mentre dal punto di vista delle aspettative sonore o referenziali (alimentate da entrambi i linguaggi) la differenza appare meno evidente. In altri termini, come un lemma acquista significato strutturale all'interno di una frase, così una nota o un accordo producono aspettative formali all'interno di una frase musicale. In entrambi i casi, esisteranno aspettative soddisfatte o non soddisfatte, capaci di creare rispettivamente sensazioni di risoluzione o sorpresa.

Attribuire importanza al livello di aspettative formali che i due tipi di linguaggio alimentano non significa però eliminare il ruolo importante giocato dalle emozioni: fattori come il tempo, intensità ed agogica di un brano musicale determinano fortemente il grado di coinvolgimento emotivo. Inoltre, la musica, in genere, presenta continui momenti di "instabilità strutturale" (concatenazioni sonore di tensione/risoluzione), che coinvolgono direttamente l'aspetto emotivo di chi ascolta. Aspettative ed emozioni sono quindi due livelli di comprensione generali di entrambi i codici: il linguaggio e la musica. La musica è un insieme di scelte ed in queste scelte risiede il suo significato:

a) si sceglie un approccio analitico al suono;

b) si scelgono associazioni individuali e culturali di significati extra-musicali;

c) si scelgono reazioni estetiche al suono.

In ottica cognitivistica e neurobiologica, si dovrà tener conto degli atti dell'ultimo Congresso Mondiale sui Fondamenti Biologici della Musica, tenutosi a New York nel maggio del 2000 e pubblicati dalla New York Academy of Sciences nel giugno del 2001. In alcuni di questi documenti si nega la possibilità di considerare la musica e il linguaggio come aventi due dimensioni separate e separabili. Le abilità cognitive specifiche per la musica e per il linguaggio si svilupperebbero in stretto collegamento e dipendenza con il training al quale la mente è stata sottoposta, determinando nascita e formazione di specifiche competenze. Inoltre queste differenziate competenze avrebbero comunque la stessa origine bio-neuronale. La diffusa convinzione secondo la quale alcuni aspetti del processo linguistico sarebbero stati gestiti di preferenza da strutture neuronali dell'emisfero destro, mentre certi aspetti del processo musicale da quello sinistro, appare imprecisa, anche se per ora non totalmente confutata. Per questi motivi, l'attuale neuroscienza si sta, tra l'altro, occupando di evidenziare le diverse elaborazioni cognitive implicate nei due processi

\footnotetext{
${ }^{91}$ Cfr. Bertirotti A., 2003, Considerazioni sull'origine e l'evoluz̨ione del suono e della musica, (in stampa) in Systema Naturae. Dai suoni della natura alla musica dell'uomo, Vol. 5. Firenze.
} 
comunicativi (linguaggio e musica), di comprenderne i meccanismi sottesi, onde localizzare nel cervello le diverse attività neuronali tipiche delle due forme.

\section{Esiste una vera e propria specificità del linguaggio?}

Storicamente la teorizzazione dell'esistenza di una vera e propria specificità del linguaggio nasce con la Grammatica Generativa elaborata negli anni settanta da N. Chomsky ${ }^{92}$ e viene poi confermata ed ampliata negli anni ottanta da J. Fodor con la sua Teoria della Mente Modulare ${ }^{93}$. Secondo Fodor, il linguaggio è una sorta di "macro modulo di calcolo" dotato di una sua propria architettura funzionale e neuronale ${ }^{94}$. Questo "macro modulo" è composto di diversi sub-moduli (elaborazione fonologica, morfologica, sintattica, semantica e pragmatica) ognuno costituente l'intero processo elaborativo. Ciascun sub-modulo è "incapsulato" all'interno della struttura generale di calcolo, in modo tale che l'elaborazione delle informazioni afferenti ad un sub-modulo non sono condivise da quelle di un altro sub-modulo. Ad esempio, il riconoscimento delle caratteristiche fonologiche di una frase si attua senza l'attivazione del sub-modulo adibito al riconoscimento sintattico di quella stessa frase. Secondo questa teoria quindi, l'elaborazione (cognitiva e neuronale) prevista nella codificazione del linguaggio è tipica per l'acquisizione di abilità specifiche e ciò accade in un sub-modulo ma non in un altro, a sua volta adibito ad un compito diverso. Un importante assunto della Grammatica Generativa recita che il linguaggio si caratterizza come specifico per il suo alto grado di elaborazione sintattica, ossia la sintassi gioca un ruolo fondamentale per la sua organizzazione. Inoltre, secondo la medesima teoria, tutte le abilità richieste dal linguaggio sono gerarchicamente organizzate all'interno del cervello.

I risultati del Congresso Mondiale di New York conducono a conclusioni del tutto estranee sia a Chomsky sia a Fodor ${ }^{95}$. Questi studi si concentrano sulla confutazione dei concetti del macro-modulo indipendente-specifico e dei sub-moduli incapsulati; rifiutano l'idea che il linguaggio sia espressione di una funzione cerebrale autonoma caratterizzata da una particolare funzionalità ed architettura neuronale e considerano il linguaggio come una parziale abilità cognitiva facente parte dei più generali processi ed elaborazioni cerebrali. Viene respinta l'idea di un cervello che nelle sue operazioni si presenti statico e a com-

\footnotetext{
${ }^{92}$ Jackendoff R., 1997, The Architecture of the Language Faculty, MIT Press, Cambridge, MA.

${ }^{93}$ Cfr. Fodor J., 1983, The Modularity of Mind, The MIT Press, Cambridge Massachusetts, trad. it. 1988, La mente modulare, Il Mulino, Bologna.

${ }^{94}$ Cfr. Molino J., 2000, op. cit.

${ }^{95}$ È necessario ricordare che esistono due validi motivi per spiegare il successo che la Teoria della Grammatica Generativa ha avuto. In primo luogo, è una teorizzazione molto chiaramente esposta ed, in secondo luogo, è del tutto compatibile con l'ipotesi modulare dell'organizzazione neuronale della mente. In effetti, il concetto secondo cui il linguaggio si organizza secondo sub-moduli cerebrali di competenze trova legittimità in tutte quelle teorie basate sulla localizzazione cerebrale delle diverse competenze cognitive. Le recenti tecniche di ricerca, basate sulla risonanza magnetica, il potenziale uditivo evocato, la magnetoencefalografia (MEG), la tomografia ad emissioni di positroni (PET), la registrazione elettrofisiologica, etc. spingono le teorie a tracciare una mappatura delle aree cerebrali coinvolte in un determinato processo computazionale. Si può ritenere che sia possibile una mappatura delle zone adibite, ad esempio, alla percezione sensoriale in genere, ma non credo che sia altrettanto facile giungere ad una mappatura ammissibile quando si tratti di individuare le zone profonde che si attivano durante l'utilizzazione del linguaggio e della musica.
} 
partimenti stagni: le unità linguistiche, esattamente come i diversi livelli di comunicazione, interagiscono fra di loro e dipendono dai dinamismi contestuali presenti nella comunicazione, oltre che dalle interazioni fra individui e culture ${ }^{96}$.

Il metodo della ricerca secondo immagine cerebrale mira a dimostrare l'attività funzionale del cervello. Questa attività si rileva con diverse tecniche di indagine: la misurazione dell'attività elettrica del singolo neurone (IR) o di una popolazione di neuroni (EEG); l'attività magnetica cerebrale accompagnata da un'attività elettrica (MEG); la misura dell'attività metabolica del cervello (PET, Positon Emission Tomography o la Functional Magnetic Resonance Imaging, fMRI).

Queste tecniche consentono di individuare quali sono le aree cerebrali che si attivano di fronte a specifiche stimolazioni, anche se resta tuttavia molto difficile comparare $i$ dati raccolti con l'acquisizione di specifiche abilità musicali e linguistiche. Lo stato attuale dei mezzi e delle ricerche evidenzia quello che potremmo chiamare il quoziente di dilatazione neuronale. In altri termini, è possibile individuare, attraverso la mappatura cerebrale se esistono e quali sono le aree adibite sia alle competenze linguistiche sia a quelle musicali: vi sono infatti studi che rivelano l'attività neuronale localizzata in presenza sia dell'ascolto delle parole sia delle note ${ }^{97}$. Ad esempio, le regioni uditive primarie del cervello reagiscono molto similmente di fronte al linguaggio parlato e all'esecuzione musicale, mentre le regioni uditive secondarie permettono l'accesso ad un ascolto più raffinato sia del linguaggio sia della musica. Il giro sovramarginale del cervello sembra essere coinvolto nella comprensione del simbolismo linguistico e nella lettura di una partitura. L'area di Broca presiede all'attività motoria in relazione all'acquisizione di capacità linguistiche, ma si attiva anche durante l'ascolto-esecuzione di un brano musicale o quando si è particolarmente coinvolti dal ritmo di un sound.

Sebbene questi risultati siano lontano dall'essere esaustivi, nondimeno sono sufficienti a dimostrare che alcune importanti aree cerebrali adibite all'utilizzo del linguaggio sono altrettanto chiaramente coinvolte nell'elaborazione di eventi musicali.

\section{Relazioni fra armonia, melodia e semantica}

Le ricerche che hanno utilizzato la tecnica PET, tomografia ad emissione di positroni, hanno evidenziato aspetti interessanti sulla decodificazione della musica. Questi studi hanno infatti rivelato che:

a) all'interno di una frase musicale si creano aspettative nei confronti di alcune frequenze specifiche, ossia gli ascoltatori si attendono di udire particolari note che siano "in stile" all'interno di una frase;

b) il grado di aspettativa frequenziale dipende in larga misura dal livello di familiarità che gli ascoltatori possiedono in riferimento a quel brano.

\footnotetext{
${ }^{96}$ Fauconnier G., 1997, Mappings in Thought and Language, Cambridge University Press, Cambridge, MA.

${ }^{97}$ Più precisamente è stato rilevata l'attivazione del solco temporale superiore sinistro, il giro medio-temporale, il giro angolare, il lobo latero-frontale sia durante l'ascolto di parole sia durante quello di note.
} 
Ciò dimostra che la musica possiede la caratteristica di stimolare negli ascoltatori forti attese, non solo emotive ma anche, in un certo senso, semantiche. Affermare che la musica abbia, all'interno di se stessa, un certo grado di semanticità non significa però conferire a questa caratteristica la stessa funzione che ha nel linguaggio. In altri termini, mentre nel linguaggio il concetto di semanticità si riferisce alla presenza di referenti oggettivi extralinguistici, nel caso della musica si può parlare di semanticità solo riferita ad un certo livello di attesa sonora. In sostanza, come esiste una logica consequenziale nell'espressione dei fonemi di una lingua, esiste una altrettanto ben riconoscibile logica sequenziale nell'espressione ed ascolto delle note, anche se in questo processo entra in gioco un sistema logico-sonoro sedimentato nell'esperienza personale-culturale degli esecutori come dei fruitori.

Esiste un particolare genere musicale più specificamente adatto a verificare le interazioni computazionali presenti nel linguaggio e nella musica: l'Opera. In effetti, nell'Opera convivono linguaggio verbale, linguaggio musicale, azione drammatica e coreografia. Tutti elementi che hanno da sempre fatto discutere musicisti e letterati sulla definizione esatta di Opera. R. Wagner (1813-1883) con l'istituzione del dramma musicale, teorizzò e praticò nella sua produzione una stretta sinergica relazione fra linguaggio e musica: "Le parole conferiscono importanza alla musica e la musica sviluppa e rinforza gli elementi del linguaggio.” Secondo Stendhal, nell'Opera la musica è invece più importante rispetto al linguaggio poiché la sua funzione è di "animare le parole".

Per determinare quale dei due aspetti, linguaggio e musica, siano "scientificamente più importanti”, ossia veicolanti l'ascolto di un'Opera, sono stati condotti studi particolari. Si sono somministrati ascolti di periodi linguistici e frasi musicali (termini e/o note) entrambi congruenti e non congruenti, nella loro parte finale. I risultati dimostrano chiaramente, a livello di elaborazione cognitiva, l'esistenza di una indipendenza fra la categorizzazione di eventi linguistici rispetto a quella di eventi musicali, specialmente nella dimensione armonica. Per agevolare la comprensione del concetto di "dimensione armonica” è forse necessaria un'esemplificazione.

Prendiamo il caso in cui vediamo un abito in tinta unita indossato da una persona. La nostra percezione dell'abito e della persona, prima di essere analitica, è sintetica, ossia percepiamo l'abito e la persona nella loro totalità, e possiamo eventualmente essere d'accordo sul gusto estetico. Prendiamo un altro caso in cui siamo di fronte ad un abito di più colori. La nostra prima percezione rimane sempre sintetica, ma interviene una spontanea valutazione dell'insieme cromatico dell'abito. In altri termini, la valutazione sintetica dipende da una precedente valutazione analitica dei colori, i quali vengono successivamente considerati nelle loro interrelazioni. Questo secondo esempio è tipico di una valutazione armonica, applicabile sia ai colori sia ai suoni.

Per tornare all'ascolto dell'Opera, i nostri processi elaborativi sono quindi di due tipi ed indipendenti fra di loro: da un lato, identifichiamo il livello semantico del linguaggio separatamente dal livello musicale e dall'altro, sembra che il linguaggio sia decodificato per primo rispetto alla musica.

Ulteriori studi tentano di verificare questa indipendenza, correlandola sia agli effetti dell'attenzione volontaria sia a quelli dell'attenzione indotta in ascoltatori musicisti di fronte 
all'Opera. L'ipotesi di fondo di queste ulteriori ricerche consiste nel ritenere che se i due aspetti (linguistico e musicale) sono indipendenti nelle loro rispettive elaborazioni, gli ascoltatori dovrebbero essere in grado di focalizzare la loro attenzione sull'uno o sull'altro, in base alle istruzioni degli sperimentatori. Senza entrare nel dettaglio, si è visto che i musicisti professionisti non decodificano il linguaggio, perché, rivolgendo la loro attenzione all'ascolto della musica, non si accorgono di eventuali incongruenze semantiche e grammaticali. Di converso, l'attenzione dei musicisti verso il significato delle parole si verifica solo nel caso in cui essi riconoscano incongruenze nella fine della frase linguistica. Questi risultati sarebbero quindi un'ulteriore conferma dell'indipendenza del linguaggio dalla musica nei loro rispettivi processi computazionali.

Esiste comunque un certo limite a questa forma di indipendenza. In effetti, un certo tipo di incongruenza presente nei dati linguistici può influenzare la percezione dei dati musicali melodici: quando le parole sono semanticamente incongruenti e cantante al di fuori dello schema tonale dell'intera opera, i musicisti rilevano solo l'incongruenza musicale, anche quando viene loro esplicitamente chiesto di concentrarsi sul linguaggio.

Gli studi fin qui esaminati erano tesi a localizzare l'interazione fra incongruenze semantiche e incongruenze musicali a livello melodico. Altri studi sono stati condotti per localizzare un altro tipo di interazione: quella tra incongruenze linguistiche e incongruenze musicali a livello armonico e contrappuntistiche ${ }^{98}$.

Armonia e contrappunto costituiscono, nelle loro interazioni reciproche, la cosiddetta grammatica della musica tonale. La sintassi è, negli atti linguistici, utile per stabilire regole che formino una struttura di significati all'interno della quale ogni parola è funzionale alla presenza dell'altra. Nello stesso modo, le regole dell'armonia conferiscono a specifici elementi musicali, note ed accordi, l'adempimento di particolari funzioni utili alla struttura del brano. In altri termini, le regole dell'armonia classica occidentale si sono sviluppate su presupposti teorici che hanno attribuito a certe combinazioni di suoni il carattere di naturalita ${ }^{99}$. Il problema scientifico attuale è quello di individuare l'effettiva naturalez:a di queste regole, ossia di individuare le basi biologico-acustiche di tali convinzioni. In ottica neurobiologica, la domanda è la seguente: esistono precise condizioni anatomiche e fisiologiche del sistema uditivo umano che, collegate al sistema cognitivo, determinano la percezione di suoni simultanei definibili come più o meno armoniosi? I risultati dimostrano che fondamentali proprietà fisiologiche ed anatomiche del sistema uditivo, unitamente al sistema cognitivo generale, veicolano la percezione di alcune sequenze di suoni simultanei

\footnotetext{
${ }^{98}$ Il contrappunto " $(. .$.$) è la teoria della disposizione e del movimento delle parti, con riguardo alla combina-$ zione tematica (...).” Schöenberg A., 1922, op. cit., pag. 14

${ }^{99}$ Nei manuali di armonia classica occidentale il concetto di naturale è associato a quello di naturalità cosmica. Nella trattatistica troviamo l'idea di "suono naturale", "scala naturale" ed "armonia naturale" in riferimento a presunte correlazioni con la natura in senso lato. Per la cultura greca classica il concetto di armonia possedeva una connotazione filosofica, ossia naturale, e matematica insieme. Per quello che concerne la presunta naturalità del suono si è fatto appello alla cosiddetta teoria degli armonici: "Le vibrazioni risonanti che si generano nella sorgente sonora in concomitanza della vibrazione fondamentale e che costruiscono i cosiddetti suoni armonici, si irradiano nello spazio e nel tempo, e tutti gli armonici hanno una curva perfettamente sinusoidale, il numero delle onde (o periodi) aumenta
} 
come più armoniose rispetto ad altre ${ }^{100}$. La percezione di maggiore o minore dissonanzaconsonanza, anche nel caso di suoni simultanei isolati, è determinata in larga misura dalle esperienze cognitivo-sonore dei soggetti. Ad esempio, la percezione di "durezza" in riferimento a certi intervalli (seconda minore, quarta, quinta e tritono) avviene all'interno di un arco di fluttuazioni che va da 20 a $200 \mathrm{~Hz}$, registrate durante l'attivazione delle fibre nervose del sistema uditivo ${ }^{101}$. Inoltre, il riconoscimento-attribuzione di consonanza e dissonanza a coppie di suoni dipende dall'attività di precisi patterns neuronali e la loro localizzazione cerebrale è identica a quella dei processi linguistici, con processi simili di polarizzazione e tempi di latenza. In pratica, i risultati dimostrerebbero la presenza di un generale processo cognitivo che si attiva in presenza sia di sequenze di suoni simultanei sia di sequenze linguistiche ${ }^{102}$, anche se si evidenzia una predominanza dell'emisfero sinistro per il linguaggio e di quello destro per la musica.

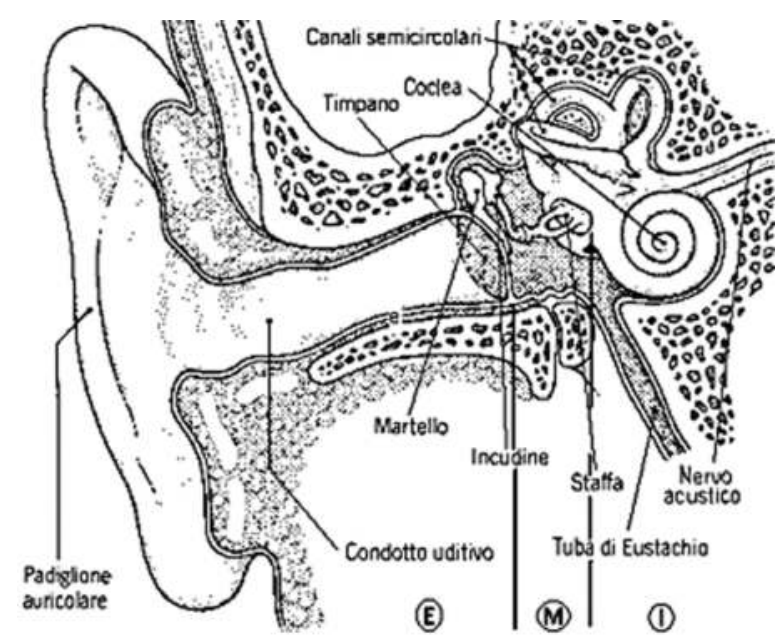

Fig. 1. E: orecchio esterno; M: orecchio medio; I: orecchio interno.

Elaborazione personale tratta da Kahle W., Leonhardt H., Platzer W., 1987, Anatomia umana Sistema nervoso e organi di senso, Vol. 3, Casa Editrice Ambrosiana, Milano.

col crescere dell'altezza dei suoni irradiati." Rognoni L., 1991, Introduzione, in Schöenberg A., 1922, Harmonielehre, op. cit., pag. 23. Ma in realtà, gli armonici (ad esempio quelli del suono do1 sono: $\mathrm{do}_{2}, \mathrm{sol}_{2}, \mathrm{do}_{3}, \mathrm{mi}_{3}, \mathrm{sol},\left(\mathrm{si} \mathrm{b}_{3}\right), \mathrm{do}_{4}, \mathrm{re}_{4}$, $\mathrm{mi}_{4}, \mathrm{fa}_{4}, \mathrm{sol}_{4}, \mathrm{ecc}$.) come suoni in sé non esistono in natura, e la teoria degli armonici è solo uno schema analitico interpretativo del suono.

${ }^{100}$ Cfr. Tramo M.J., Cariani P.A., Delgutte B., Braida L.D., 2001, Neurobiological Foundations for the Theory of Harmony in Western Tonal Music, in The Biological Foundations of Music, Annales of the New York Academy of Sciences, vol. 930 , New York, pagg. 92-113.

${ }^{101}$ I teorici medievali hanno sempre manifestato la loro avversione per i suoni cosiddetti "duri". La quarta eccedente, il tritono, cioè l'intervallo fa-si naturale, era ritenuto ad esempio di effetto sgradevole e duro, perché di difficile intonazione: "Si contra fa est diabulus in musica".

${ }^{102}$ La teoria a monte di questi risultati fu elaborata già da D’Andrade, nel 1950. Egli sosteneva che “(...) culture is an mental phenomenon (...) [e] the structures that exist in physical world as objects or events (...) are all thought to $(. .$.$) be more or less a reflection of these mental cultural structures." D’Andrade R., 1996, The Development of$ Cognitive Anthropology. Cambridge University Press, Cambridge, UK, pag. 146. 
La dimensione temporale ${ }^{103}$ del codice linguistico e di quello musicale merita diverse ed altrettanto importanti considerazioni. Il problema della percezione dinamica del tempo è stato al centro dell'attenzione scientifica per molti anni e ancora oggi sono molte le interpretazioni in merito.

Ottenere una precisa o generale definizione di timing è di solito un compito difficile, anche perché molti aspetti del termine sono dati per scontati dalla maggior parte delle persone. Esiste comunque una differenza generale macroscopica fra un tempo personale ed un tempo pubblico, ossia fra la sensazione-percezione interna del trascorrere della vita e la percezione-accettazione di un tempo ufficiale che regola impersonalmente alcuni aspetti della vita quotidiana. Il tempo personale è quindi sempre interno, variabile e soggettivo, mentre quello pubblico è esterno, meccanico e invariabile. Qualsiasi musicista è consapevole di questa doppia valenza temporale, infatti legge o riconosce le indicazioni temporali della partitura anche se spesso non ne tiene conto. Infatti a volte si ascolta lo stesso brano eseguito da diverse orchestre o solisti a velocità diverse e non si ha la percezione della differenza. Nello stesso tempo, i musicisti sperano di non impiegare la stessa velocità nella riesecuzione di un brano, proprio in nome di una dinamismo cognitivo-temporale che fa appello alla vita personale di ognuno di loro. Ciò che è comunque sorprendente è che gli studi effettuati sul timing evidenziano una quasi totale non consapevolezza da parte del musicista dei propri meccanismi temporali. La comprensione conscia del timing non è assolutamente necessaria all'esecuzione formale di un pezzo e comunque non fa parte della sua preparazione: il musicista opera all'interno di una continua ri-creazione del brano a quattro dimensioni (spazio-tempo nella verticalità dell'armonia e spazio-tempo nella orizzontalità della melodia). Questo è possibile grazie ad un tipo di notazione che appartiene solo alla musica ${ }^{104}$.

Qualche esempio può essere utile: il musicista opera una continua e simultanea trasposizione mentale, affatto complicata, dal piano visivo della partitura al piano esecutivo lungo un continum spazio-temporale. Egli decodifica le note situate in una simultanea dimensione, ed ogni movimento esecutivo si dispiega lungo il timing generale del brano, ossia durante tutta la durata dell'esecuzione. Se utilizziamo il pianoforte come ulteriore esempio pratico, la lettura verticale delle note contemporanee viene eseguita su un piano orizzontale (la tastiera), con le note scritte nella parte superiore delle linee del pentagramma corrispondenti sulla tastiera ai tasti del lato destro.

${ }^{103}$ Nella lingua inglese questa dimensione è definita timing, e non possiede una precisa definizione nella lingua italiana. Il timing sta comunque ad indicare la capacità di inviare messaggi o compiere delle operazioni secondo una particolare disposizione dei fenomeni lungo uno spazio temporale.

${ }^{104}$ In tutto il Medioevo la maggior parte dei musicisti eseguiva musica senza l'ausilio della notazione. Lo sviluppo della notazione ha favorito ovviamente la sua diffusione: dal 1476, con la stampa dei canti gregoriani e dal 1501 con la pubblicazione di opere polifoniche. Le prime forme di notazione designavano solamente l'altezza dei suoni e solo parzialmente il loro valore temporale. La notazione neumatica rappresenta il primo tentativo di indipendenza della scrittura rispetto alla tradizione orale e, nello stesso tempo, di ottenere una certa precisione. In questo modo si sono sviluppate dal neuma due tipi di notazione: quella inscritta in partiture simili ad una mappa geografica di suoni e quella che utilizzava le lettere o numeri indicanti le posizioni delle dita sullo strumento od il modo di esecuzione. Questo sforzo notazionale porterà alla partitura tipica del XVI secolo. Nasce quindi l'opera d'arte musicale, intesa come composizione interamente scritta e quindi frutto di una dottrina compositiva delineata. 
Prométhée.

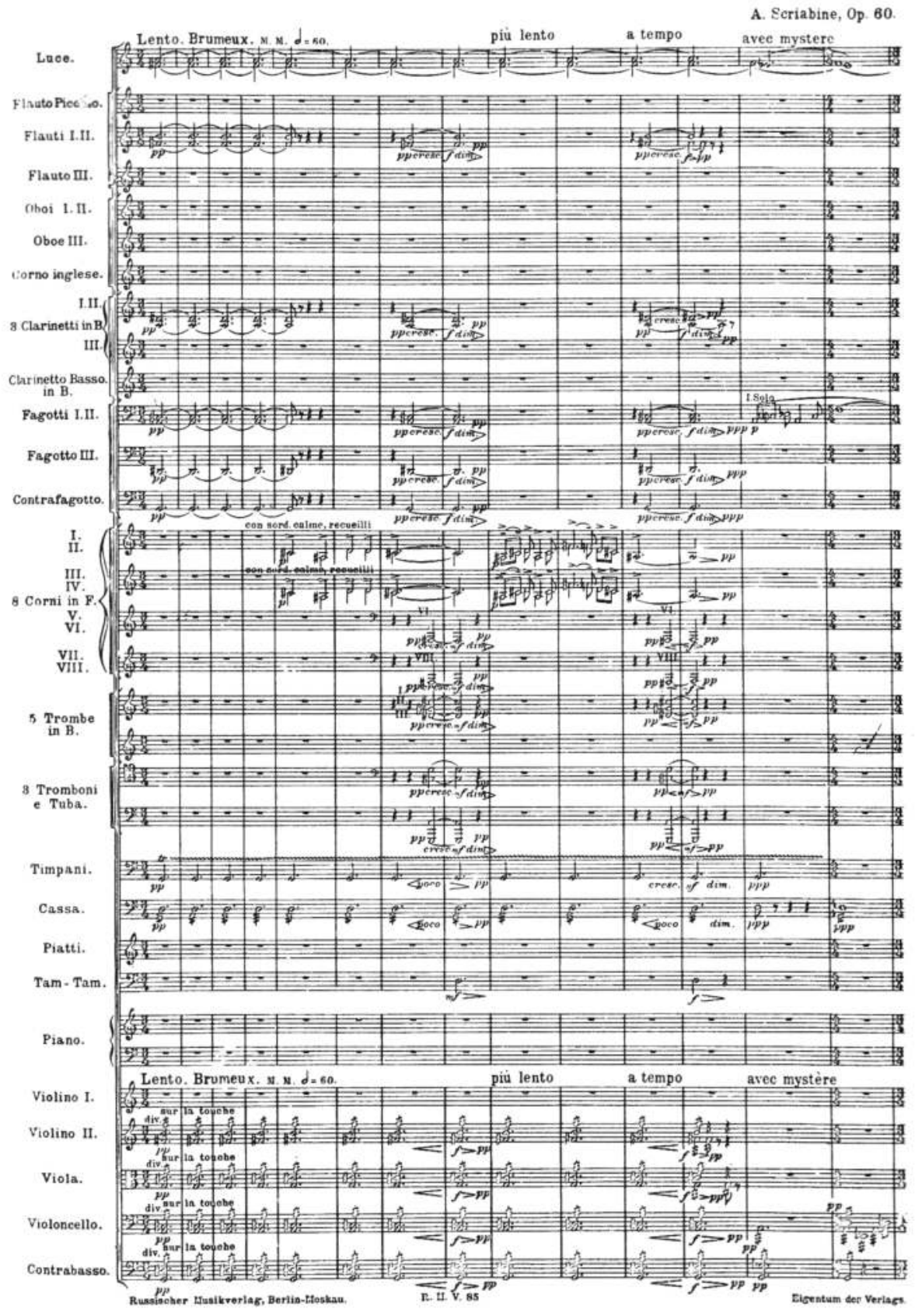

Fig. 2. Inizio della partitura del Prometeo di A.N. Skrjiabin. 
Come si può notare nella Fig. 2, la pagina è una forma unica di scrittura spazio-temporale quadridimensionale e la sua "messa in esecuzione" è frutto di un'utilizzazione assai raffinata e complessa del sistema nervoso. Dal punto di vista neurologico, tutti i musicisti si allenano nel controllo costante delle vibrazioni, affinché si presentino ad impulsi regolari al secondo. Qualunque sia lo strumento o la lunghezza del brano, il musicista deve produrre dei suoni ad una precisa frequenza, al momento giusto, sia del tempo interno sia di quello esterno: possiede quindi un controllo delle vibrazioni, fatta eccezione degli strumenti ad accordatura fissa.

Secondo Carolyn Drake e Daisy Bertrand ${ }^{105}$, lo studio della percezione del tempo musicale potrebbe rivelarsi come utile ausilio allo studio di meccanismi percettivi e cognitivi più generali.

Abbiamo visto che quando si ascolta o si produce personalmente della musica, ciascun evento (sequenza di suoni disposti a gradi congiunti o ad intervalli consonanti e dissonanti; pulsazioni ritmiche e metriche; fraseggio; dinamica e qualità del suono, etc.) deve essere collocato e rappresentato nella nostra mente all'interno di un continum temporale di eventi musicali circostanti, e decodificato in relazione a questi ${ }^{106}$. Le strutture temporali presenti nella musica, patrimonio universale dell'uomo, variano da cultura a cultura. In passato, si è teorizzato che il livello di elaborazione temporale presente nella musica (quindi nel compositore, esecutore e fruitore) fosse direttamente proporzionale alle diverse esperienze individuali ed al livello di integrazione culturale. Le ricerche attuali invece hanno dimostrato che esistono strutture cognitive adibite alla decodificazione dei processi temporali indipendenti dalla cultura di appartenenza e dall'esperienza. Si dovrebbe quindi parlare di un vero e proprio universale biologico. Inoltre, la percezione del ritmo da parte di adulti musicisti, non musicisti, bambini e neonati sembra svilupparsi indipendentemente dal grado di educazione musicale. Carolyn Drake e Daisy Bertrand hanno infatti individuato quattro tipologie "universali" di elaborazione del tempo musicale ,che potrebbero essere utilizzate come paradigmi di ricerca dall'etnomusicologia. Vediamo nel dettaglio.

\section{Segmentazione e raggruppamento}

Questo modello cognitivo temporale si manifesta nella tendenza a raggruppare all'interno di unità percettive singole gli eventi che presentano caratteristiche fisiche simili, o che si realizzano spesso nell'arco di un preciso periodo di tempo (vedi Fig. 3). Ogni individuo esprime sempre la necessità di controllare gli eventi temporali, inserendoli in un comprensibile processo dinamico. Questa necessità, funzionale alla percezione generale del tempo, si manifesta in rappresentazioni continue e ristrette di raggruppamenti (vedi le

${ }^{104}$ Drake C., Bertrand D., 2001, The Quest for Universals in Temporal Processing in Music, in Zatorre R.J. , Peretz I., (a cura di), June 2001, op. cit.

${ }^{105}$ Ad esempio, quando alcuni musicisti professionisti decidono di suonare assieme (in concertazione), indipendentemente dal loro numero, si assoggettano alla disciplina di un "direttore d'orchestra". Il direttore, utilizzando una serie complessa di gesti (anche con l'ausilio della bacchetta) compie una divisione dello spazio (una specie di "vibrazione spaziale"). Il direttore è in grado di dividere lo spazio ed il tempo per un numero vasto di strumenti, affinché essi producano le loro tipiche vibrazioni attraverso diverse sequenze di movimenti corporei e tutti in relazione alla notazione. 
unità percettive della Fig. 3) di eventi temporali singoli (le lettere e le relative note in notazione anglosassone delle cinque unità percettive), all'interno di un dinamismo più ampio. In altri termini, la nostra mente, di fronte ad ulteriori eventi percettivi, opera una sorta di insistita comparazione con eventi percettivi già presenti nella memoria personale e al tempo stesso predittiva rispetto a questi. Un evento originale e completamente nuovo che si presenti alla mente verrà assimilato (l'unità percettiva n. 5 della Fig. 3).

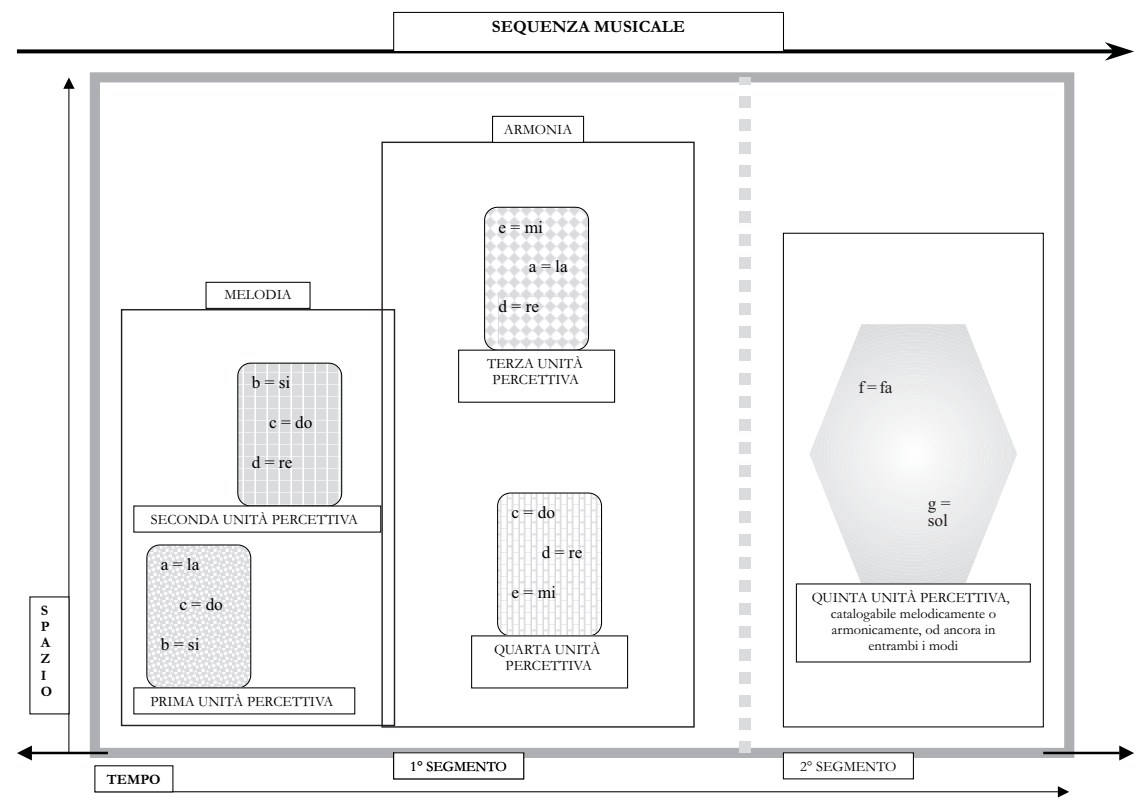

Fig. 3. Schema del funzionamento delle unità percettive

Nel caso in cui questo evento sia invece troppo diverso (nei suoi aspetti acustici o temporali) dagli eventi già memorizzati (sempre l'unità percettiva n. 5 della Fig. 3), la sequenza percepita verrà opportunamente segmentata. In sostanza, tutti gli elementi che possono far parte di un unico insieme saranno elaborati come un tutto unico e all'interno di singole unità percettive.

Sono molti gli studi che dimostrano l'esistenza di questo processo cognitivo. La Gestaltpsychologie, ad esempio, ha rivelato che gli ascoltatori segmentano le sequenze di suoni secondo macrocaratteristiche (timbro, intensità, altezza, durata, pause, etc). Il cambiamento di una sola di queste macro caratteristiche conduce ad una rottura di continuità percettiva e alla creazione di microcaratteristiche percettive. Ad esempio, la successione di suoni disposti a gradi congiunti (come le scale in genere e per esempio la scala di sol maggiore: sol la do re mi fa\# sol) viene elaborata secondo il principio dell'altezza (macrocaratteristica o categoria interpretativa). Una qualsiasi variazione in questa successione conduce ad una improvvisa rottura (segmentazione) della sequenza percettiva a vantaggio di una nuova unità percettiva, ad esempio la disposizione a gradi disgiunti (come una scala pentatonica ad intervalli disgiunti: sol si re fa\# sol). 
Secondo Carolyn Drake e Daisy Bertrand ${ }^{107}$ la segmentazione e raggruppamento percettivo sono un universale percettivo temporale. Le loro ricerche hanno infatti dimostrato che questo tipo di elaborazione è:

a) indipendente dal grado di educazione musicale dell'ascoltatore e si presenta sia in adulti musicisti sia in adulti non-musicisti, sebbene i primi siano più sistematici nelle loro risposte;

b) i neonati di 6-8 mesi reagiscono all'altezza ed al timbro allo stesso modo degli adulti, sebbene i primi riconoscano meglio intervalli più ampi rispetto agli adulti;

c) bambini dai 4 ai 12 anni percepiscono molto chiaramente cambiamenti di altezza, intensità, durata e pause, operando relative segmentazioni percettive ${ }^{108}$.

\section{La predisposizione alla regolarità}

L'elaborazione delle sequenze musicali è ottimale in presenza di regolarità interne alla stessa sequenza e le sequenze irregolari vengono tendenzialmente percepite come regolari.

Il nostro sistema di codificazione percettiva delle sequenze opera una continua comparazione fra i nuovi intervalli e quelli già memorizzati nell'esperienza, senza calcolare la precisa durata di ciascun intervallo. Ad esempio, se un nuovo intervallo risulta simile in durata a precedenti intervalli (all'interno di una "tolerance window") verrà catalogato come identico a questi. Invece, se la durata del nuovo intervallo è significativamente diversa dai precedenti, al di là della "tolerance window", sarà percepito come diverso. In questo contesto è importante ricordare che la capacità di codificare la durata di un intervallo come "più lunga" o "più corta" non è un procedimento assoluto. In altri termini, noi possediamo in genere due o tre categorie di differenziazione (stesso/diverso, stesso/più lungo/più corto) attraverso le quali decodifichiamo e compariamo fra loro eventi musicali diversi. Grazie alla "tolerance window", una successione irregolare di intervalli (ossia composta di diversi valori di durata) viene decodificata come regolare, ossia percepita come unitaria, mentre la percezione della diversità dipende dal grado di alterazione della velocità: nel caso in cui si modifichi la velocità di una sequenza in modo considerevole, l'ascoltatore percepirà tale modificazione; nel caso in cui la modificazione della velocità sia minima, la differenza non verrà percepita.

La percezione della regolarità è, secondo Carolyn Drake e Daisy Bertrand un universale percettivo temporale. Infatti la "tolerance window" è riscontrabile:

a) in ascoltatori musicisti e non musicisti;

b) in bambini di quattro anni in poi $^{109}$;

c) in neonati da due mesi in poi, anche se in questo caso ci si riferisce alla "capacità di reazione" di fronte alla novità.

\footnotetext{
${ }^{107}$ Cfr Drake C., Bertrand D., 2001, op. cit.

${ }^{108}$ Le autrici notarono anche un leggero miglioramento delle prestazioni in relazione all'aumento dell'età e dell'educazione musicale di base.

${ }^{109}$ Questa capacità è riscontrabile nei bambini solo nel caso in cui si tratti di una alterazione in sequenze isocrone.
} 


\section{La ricerca attiva della regolarità}

Qualsiasi ascoltatore spontaneamente ricerca delle regolarità temporali e organizza gli eventi musicali all'interno della regolarità percepita. Questa ricerca attiva si presenta quindi come una "tecnica economica" di decodificazione musicale, in grado di ottimizzare la comprensione della sequenza. Si tratta di una organizzazione ergonomica attuata dall'ascoltatore, affinché un unico principio assimilatore (appunto la regolarità) unifichi in un unico processo dinamico i diversi aspetti (ossia qualità) della sequenza musicale.

Questo tipo di organizzazione temporale è facilmente osservabile. A tutti noi è accaduto di battere il tempo (con le mani o con il piede) ascoltando un brano che presentasse regolarità ritmiche o "irregolarità irregolari". La nostra reazione agli impulsi regolari (in musica meglio definiti accenti forti) di un ritmo accattivante non è solo fisiologica, ma cognitiva, ossia legata al miglioramento della percezione temporale del brano stesso.

Carolyn Drake e Daisy Bertrand hanno evidenziato che la ricerca attiva di regolarità ritmiche presenti in una sequenza musicale è un universale percettivo temporale. Infatti si riscontra

a) in musicisti e non musicisti per più del $90 \%$ dei casi, anche se il brano contiene molte microvariazioni di accenti;

b) in tutti i soggetti di età compresa fra i 6/10 anni e 24, i quali sono in grado di sincronizzare il loro "battere il tempo" con l'inizio del Bolero di Ravel;

c) nei neonati, i quali conformano il loro ritmo di suzione con quello di un brano che stanno ascoltando.

\section{Il livello temporale ottimale di catalogazione degli eventi sonori}

Quando si ascoltano sequenze musicali si tende a catalogare gli eventi sonori di cui sono composte all'interno di un "spazio percettivo ottimale e intermedio". In altri termini, Carolyn Drake e Daisy Bertrand hanno dimostrato che la sensibilità alla percezione di cambiamenti (ritmici, di durata e di intervalli) è vincolata ad un "range" percettivo superiore ai 6 decimi di secondo.

Questo paradigma percettivo è particolarmente importante perché sperimentato esclusivamente nei confronti di adulti non musicisti ed evidenzia come, in certe produzioni musicali di largo consumo, le variazioni ritmiche siano spesso macroscopiche e funzionali alla loro stessa percezione.

In conclusione, queste ricerche sono indubbiamente importanti e suscitano un notevole interesse, anche se riteniamo siano necessarie ulteriori ricerche etnomusicologiche che eventualmente giungano agli stessi risultati, evidenziando cioè gli stessi meccanismi di segmentazione e raggruppamento, indipendentemente dalla cultura di appartenenza. Solo allora sarà possibile ipotizzare l'esistenza di universali musicali a base biologica e cognitiva.

A questo punto siamo in grado di valutare più agevolmente il rapporto fra il concetto di tempo in musica e nel linguaggio: in entrambi si assiste ad un dispiegamento temporale di eventi acustici. Proprio a causa di questa reciproca struttura temporale, eventi specifici appartenenti ad entrambi i codici, ossia i suoni (note e fonemi), rispettano precise regole temporali, ad esempio quelle evidenziate da Carolyn Drake e Daisy Bertrand. Quindi, è 
importante verificare sperimentalmente se i processi tipici della dimensione temporale di entrambi gli atti (linguistico e musicale) possano collegarsi ad un generale meccanismo cognitivo temporale o differiscano in funzione del materiale che caratterizza i due processi. Le ricerche sono state condotte utilizzando sia il metodo ERP (event-related brain potentials) sia il MEG (magneto-encephalography) ed hanno evidenziato il periodo temporale di riconoscimento di violazioni all'interno di strutture temporali linguistiche e musicali. Ulteriori studi hanno utilizzato la fMRI (functional magnetic resonance imaging), la quale a sua volta ha permesso di localizzare le strutture cerebrali che si attivano in presenza delle suddette violazioni. L'ipotesi di fondo affermava che qualora fossimo stati in presenza di un meccanismo di elaborazione temporale congiunto, i risultati rilevati con l'ERP e il MEG sarebbero stati qualitativamente simili. Inoltre, altrettanto simili sarebbero state le rivelazioni eseguite con l'fMRI. Al contrario, se l'elaborazione temporale delle informazioni di entrambi i sistemi avesse fatto appello a diverse strutture temporali, si sarebbero individuati diversi risultati con l'ERP, il MEG e l'fMRI.

Le ricerche hanno rilevato che esiste una stretta correlazione fra l'ERP ed il livello di attesa sonora, sia per i musicisti sia per i non-musicisti. In altri termini, somministrando una frase musicale nella quale era stata introdotta una pausa fra la penultima e l'ultima nota della frase stessa, gli ascoltatori restavano particolarmente sorpresi di questa variazione.

Questo metodo è stato applicato anche nel caso delle aspettative sonore linguistiche. Sono state quindi somministrate frasi familiari (ad esempio i "detti" o i "proverbi") in contrapposizione a frasi non-familiari. In metà delle frasi familiari e non-familiari sono stati presentati termini nelle loro originarie posizioni sintattiche, mentre nelle altre metà erano ritardati di $600 \mathrm{~ms}$. L'ERP ha dimostrato una emissione di potenziale nel caso in cui i termini erano ritardati rispetto alle posizioni originali, sia per le frasi familiari sia per quelle non-familiari.

In sostanza, le rilevazioni effettuate hanno dimostrato una stretta correlazione fra il livello di aspettativa sonora e l'alterazione dell'ERP, sia nel caso degli eventi sonori musicali sia linguistici. Tutti questi risultati dimostrano che la decodificazione di sequenze sonore nella loro successione cronologica (processing temporal information), sia nel caso di sequenze linguistiche sia musicali, si fondano su generali meccanismi cognitivi.

\section{Conclusioni}

In questo capitolo si è inteso individuare eventuali correlazioni fra capacità cognitive e apprendimento linguistico e musicale. Si impongono due domande: a) il linguaggio e la musica si fondano su sistemi cognitivi autonomi, indipendenti dalle altre abilità cognitive, oppure sono direttamente collegate e collegabili all'acquisizione di abilità cognitive generali ed universali? b) il linguaggio e la musica sono tra loro indipendenti nelle loro elaborazioni cognitive?

Nel tentativo di ripercorrere lo stato attuale della ricerca in questo settore si sono presi in esame una serie di risultati del Congresso Mondiale tenutosi a New York nel maggio del 2000, avente come tema The Biological Foundations of Music, pubblicato dalla New York Academy of Sciences nel giugno di quest'anno, che comparano il sistema di elaborazione linguistica con quello di elaborazione musicale. 
Da questi risultati, emerge innanzi tutto che ogni tipo di elaborazione semantica richiede l'accesso al significante e al significato degli eventi sonori (fonemi e note), e che questo accesso è veicolato dal contesto temporale (sintassi) in cui gli stessi eventi occorrono. Questo accesso è frutto di scelte cognitive, e lo studio di queste scelte appare destinato a favorire la comprensione dei meccanismi cerebro-mentali che le presiedono.

La presenza di questo strutturato complesso di preferenze dimostrerebbe la validità della Teoria Modulare della Mente (MMT) e delle competenze di domini cognitivi specifici, senza collegarsi direttamente ad un disegno evolutivo preciso. La realizzazione effettuata nel corso di questi studi di una mappatura delle aree cerebrali adibite al linguaggio e alla musica ha evidenziato l'intervento, autonomo e indipendente, di specifiche popolazioni neuronali, nell'uno o nell'altro caso, ed è probabilmente in questa direzione che dovranno svilupparsi gli studi futuri.

Questo settore di indagine dovrà probabilmente essere corroborato da ulteriori ricerche etnomusicologiche, in grado di fornire una interpretazione culturologica di alcuni meccanismi cognitivi dotati di universalità.

Abbiamo trattato, a questo proposito, della tendenza al raggruppamento e segmentazione delle sequenze di suoni, che è indipendente dalla cultura di appartenenza e quindi tratto cognitivo universale.

Per quello che concerne l'indipendenza della musica dal linguaggio, si è visto che le ricerche condotte evidenziano l'intervento di meccanismi complessi per entrambi i codici comunicativi. Questi codici si differenziano innanzi tutto per il grado di semanticità, referenziale nel caso del linguaggio ed auto-referenziale nel caso della musica, mentre aspettative ed emozioni caratterizzano entrambi. Le indagini condotte hanno rilevato che le acquisizioni di abilità linguistiche e musicali dipendono dal training formativo al quale la nostra mente è sottoposta, sebbene il patrimonio neuronale implicato in queste acquisizioni sia identico.

La questione delle paternità neuronale dell'emisfero sinistro e del destro è stata studiata utilizzando il genere musicale dell'Opera, proprio in virtù della presenza in esso sia del linguaggio sia della musica. I dati hanno dimostrato, attraverso lo studio delle violazioni sintattiche in entrambi i codici, che linguaggio e musica (nella sua dimensione melodica, armonica e contrappuntistica) sono il frutto di elaborazioni cognitive indipendenti, anche se le strutture neuronali coinvolte sono identiche per alcuni fattori, ad esempio per il timing.

Al di là degli specifici settori di ricerca, appare individuato un cammino in cui la moderna neuroscienza si dedichi con sempre maggiore impegno anche alla cognizione musicale, con l'obiettivo di collocare l'assunzione di abilità cognitive musicali in un schema interpretativo più ampio, che tenga conto dei diversi livelli di plasticità cerebrale. In effetti, è proprio questo ultimo concetto che lega indissolubilmente le acquisizioni di abilità musicali e del linguaggio a quelle di abilità cognitive generali. 



\section{Bibliografia}

Aiello R., 1994, Music and language: parallels and contrasts, in Aiello R. \& Sloboda J., Musical perception, Oxford University Press, New York.

Arom S., 2000, Prolegomeni to a biomusicology, in Wallin N.L., Merker B., Brown S., 2000, (eds), The Origins of Music, MIT Press, Cambridge, Mass.

Balbi R. e R., 1982, Lungo viaggio al centro del cervello, Edizione Clueb su licenza G. Laterza, Bari.

Barkow J., Cosmides L., Tooby J., 1992, The Adapted Mind: Evolutionary Psychology and the Generation of Culture, Oxford University Press, Oxford.

Bertirotti A., 2003a, L'uomo, il suono e la musica, Firenze University Press, Firenze.

Bertirotti A., 2003b, Considerazioni sull'origine e l'evoluzione del suono e della musica in Systema Naturae. Dai suoni della natura alla musica dell'uomo, vol. 5, (in stampa).

Bertirotti A., 2003c, Il tempo nella musica. Considerazioni sull'andamento musicale, Firenze University Press, Archivio E-Print, Firenze.

Blacking J., 1973, How Musical is Man?, University of Washington Press, Seattle.

Bowling W.J., 1978, Scale and contour: two components of a theory of memory for melodies, in Psychological Review, 85, trad. it. Lorenzetti L.M., Antonietti A. 1986, I processi cognitivi nella musica, in Processi cognitivi in musica, (a cura di), Franco Angeli Editore, Milano.

Castelli P.A., 1992, Il bambino, i suoni e la musica, Edizioni Centro Ipes, Milano.

Chang H., Trehub S.E., 1977, Auditory processing of relational informations by young infants, in "J. Exp. Child Psychol.", 24; Infant's perception of temporal grouping in auditory patterns, in "Child Development", 1977, 48.

Chiarelli B., 1997, Evoluzione del cervello e nascita dell'autocoscienza, in Systema Naturae. L'uomo, la natura e il pensiero, vol. 4, Edizioni Polistampa, Firenze.

Chomsky N., 1969-1970, Aspects of the Theory of syntax, Cambridge, Mas., MIT Press, trad. it. Aspetti di una teoria della sintassi, Boringhieri, Torino.

Chomsky N., 1970, Syntactic structures, The Hague, Mouton, trad. it. Le strutture della sintassi, Laterza, Bari.

Chomsky N., 1971, Language and mind, New York, Harcourt Brace Jovanovitch.

Critchley MacDonald \& Henson R.A., 1987, Music and the Brain. Studies in the Neurology of Music. William Heinemann Medical Books Limited, London, trad. it. La musica e il cervello. Studi sulla neurologia della musica, 1987, Piccin Editore, Padova.

Cross I., 2001, Music, Cognition, Culture, and Evolution in The Biological Foundations of Music, Annales of the New York Academy of Sciences, vol. 930, New York.

D'Andrade R., 1996, The Development of Cognitive Anthropology, Cambridge University Press, Cambridge, UK.

Delalande F., 1993a, Le condotte musicali. Comportamenti e motivazioni del fare musica e ascoltare musica, Editrice Clueb Bologna.

Delalande F., 1993b, Che cosa è la musica?, in Il senso della musica. Semiologia della musica., (a cura di) Ferrari F., Stefani G., Dispensa del Corso quadriennale di Musicoterapia, Centro di Educazione Permanente, La Cittadella, Assisi. 
De la Motte-Haber H., 1972, Psicologia della musica, Discanto Edizioni.

Deliège I., Sloboda J., 1997, Perception and Cognition of Music, The Psychology Press, Hove. Drake C., Bertrand D., 2001, The Quest for Universals in Temporal Processing in Music, in Zatorre R.J., Peretz I., (a cura di), June 2001, Biological Foundations of Music, Annales of the New York Academy of Sciences, vol. 930, New York.

Durand G., 1963, Les structures anthropologiques de l'Imaginaire, Presses Universitaires de France, trad. it. 1972, Le strutture antropologiche dell'immaginario, Edizioni Dedalo.

Eco U., 1962, Opera aperta, Bompiani, Milano.

Fauconnier G., 1997, Mappings in Thought and Language, Cambridge University Press, Cambridge, MA.

Ferchault G., 1957, Mélodie, in Larousse de la musique, Larousse, Paris.

Fodor J., 1983, The Modularity of Mind, The MIT Press, Cambridge Massachusetts, trad. it. 1988, La mente modulare, Il Mulino, Bologna.

Gardner H., Wolf D., 1995, The waves and streams of symbolization, in Acquisition of symbolic skills, (a cura di) Rogers e J. A. Sloboda, New York, Plenum.

Geertz C., 1973, The Interpretation of Cultures: Selected Essays, Basic Books, New York.

Giuntini G., 2001, Gli utensili del musicista, in Suoni della terra. Forme musicali, Catalogo della mostra, Fondazione Carlo Marchi, Firenze.

Jackendoff R., 1997, The Architecture of the Language Faculty, MIT Press, Cambridge, MA.

Kahle W., Leonhardt H., Platzer W., 1987, Anatomia umana - Sistema nervoso e organi di senso, Vol. 3, Casa Editrice Ambrosiana, Milano.

Károly O., 1965, Introducing Music, Penguin Books Ltd, Harmondsworth, Middlesex, trad. it. 1969, La grammatica della musica. La teoria, le forme e gli strumenti musicali, Piccola Biblioteca Einaudi, Einaudi Editore, Milano.

Langer J., 1969, Theories of Development, Holt Rinehart and Winston Inc., New York, trad. it. 1973, Teorie dello sviluppo mentale, Giunti Barbera, Firenze.

Langer S.K., 1975, Feeling and Form, trad. it. di Lia Formigari, Feltrinelli Editore, Milano.

Leroi-Gourhan A., 1977, Il gesto e la parola, Einaudi, Torino.

Leroi-Gourhan A., 1981, I più antichi artisti d'Europa, Jaca Book, Milano.

Leroi-Gourhan A., 1986, Meccanica vivente, Jaca Book, Milano.

Lorenzetti L.M., 1985, Il suono nell'unità mente-corpo: funzioni preventive in ambito psicomotorio e nel ritardo mentale, in Disturbi dell'apprendimento e musica, Pro Civitate Christiana, Assisi.

Lorenzetti L.M., Antonietti A. 1986, I processi cognitivi nella musica, in Processi cognitivi in musica, (a cura di), Franco Angeli Editore, Milano.

Mead G., 1962, Mind, Self and Society, The University of Chicago Press, Chicago, trad. it. 1966, Mente, Sé e Società, Giunti Barbera, Firenze.

Molino J., 2000, Toward an evolutionary theory of music and language, In Wallin N.L., Merker B., Brown S., 2000, (eds), The Origins of Music, MIT Press, Cambridge, Mass.

Morino Abbele, F., 1984, Introduzione alla psicologia sociale, Giunti Barbera, Firenze.

Merton Robert K., 1968, Social Theory and Social Structure, The Free Press, New York, trad. it. 1970, Teoria e struttura sociale, vol. I, Il Mulino, Bologna.

Musio G., 1989 (5a ed.), Antropologia e mondo moderno, Franco Angeli, Milano. 
Musio G., 1995, La mente culturale. Struttura della cultura e logica della organizzazione della mente, Università di Firenze.

Mussen P.H., Conger J.J., Kagan J., Child Development and Personality, trad. it. 1981, Lo sviluppo del bambino e la personalità, Zanichelli Editore, Bologna.

Nattiez J.J., 1987, Il discorso musicale. Per una semiologia della musica, Einaudi Editore, Torino.

Nettl B., 1984, The study of ethnomusicology, University of Illinois Press.

Pacherie E., Philosophie et sciences cognitives, in Encyclopédie Philosophique Universelle. Mattei J.F., Edizioni PUF, Paris.

Piaget J., 1945, La formation du Symbole chez l'enfant, Delachaux et Niestlé, Neuchâtel, trad. it. 1972, La formazione del simbolo nel bambino, La Nuova Italia Editrice, Firenze.

Postacchini P.L., 1983, Dalla percezione sonora allintervento globale su bambini con problemi, in Autismo, psicosi infantili e musica, Pro Civitate Christiana, Assisi.

Rameau P., 1743, Trattato dell'armonia ridotta ai suoi principi naturali, Bologna.

Révész G., 1954, Einführung in die Musikpsychologie, A. Francke Ag. Verlag, Bern, trad. it. 1983, Psicologia della musica, Giunti Barbera, Firenze.

Righini P., 1970, L'acustica per il musicista. Fondamenti fisici della musica, Edizioni Zanibon, Padova.

Rousseau J.J., 1781/1993. Essai sur l'origine des langues, Flammarion, Paris.

Saveur J., 1701, Principi d'acustica e di musica ovvero sistema generale degli intervalli dei suoni e sua applicazione a tutti $i$ sistemi ed istrumenti di musica, Roma.

Shepard R.N., 1992, The Perceptual organization of colours: an adaptation to regularities of the terrestrial world?, in Shepard R.N., 1992, The Perceptual organization of colours: an adaptation to regularities of the terrestrial world?, in Barkow J., Cosmides L., Tooby J., The Adapted Mind: Evolutionary Psychology and the Generation of Culture, Oxford University Press, Oxford.

Schneider M., 1960, Le róle de la musique dans la mythologie et les rites des civilisations non européennes, Éditions Gallimard, Paris, trad. it. 1992, La musica primitiva, Adelphi Editore, Milano.

Schön A. e D., 1999, Il potere del suono e della musica. Fuga a più voci, Psiche 1-2.

Schöenberg A., 1922, Harmonielehre, Universal Editing, Wien, trad. it 1991, Manuale di armonia, Il Saggiatore, Mondatori, Milano.

Schöenberg A., 1939, La composizione con dodici suoni, Atti conferenziali.

Schöenberg A., Mann T., 1950, A proposito del Doctor Faustus, Rosellina Archinto, Milano.

Sloboda J.A., 1985, The Musical Mind. The Cognitive Psychology of Music, Oxford University Press, trad. it. La mente musicale. Psicologia cognitiva della musica, Società editrice il Mulino, Bologna.

Strawinskij I., 1971, Cronache della mia vita, Universale Economica Feltrinelli.

Tramo M.J., Cariani P.A., Delgutte B., Braida L.D., 2001, Neurobiological Foundations for the Theory of Harmony in Western Tonal Music, in The Biological Foundations of Music, Annales of the New York Academy of Sciences, vol. 930, New York.

Treitler L., 1980, History, criticism and Beethoven's Ninth Symphony. Nineteenth Century Music 3. Trehub S. E., Schellenberg G., Hill, D., 1997, The origins of music perception and cognition: a development perspective, in Deliège I., Sloboda J., Perception and Cognition of Music, The Psychology Press, Hove. 
Wallin N.L., Merker B., Brown S., 2000, (eds), The Origins of Music, MIT Press, Cambridge, Mass.

Werner H., 1948, Comparative Psychology of Mental Development, International Universities Press, Inc., New York, trad. it. 1970, Psicologia comparata dello sviluppo mentale, Giunti Barbera, Firenze.

Wishart T., Musical writing, musical speaking, in Shepherd J., Virden P., Vulliamy A., Wishart G. e T., 1977, Whose music? A sociology of musical language, London, Latimer. 
1. Brunetto Chiarelli, Renzo Bigazzi, Luca Sineo (a cura di), Alia: Antropologia di una comunità dell'entroterra siciliano

2. Vincenzo Cavaliere, Dario Rosini, $D a$ amministratore a manager. Il dirigente pubblico nella gestione del personale: esperienze a confronto

3. Carlo Biagini, Information technology ed automazione del progetto

4. Cosimo Chiarelli, Walter Pasini (a cura di), Paolo Mantegazza. Medico, antropologo, viaggiatore

5. Luca Solari, Topics in Fluvial and Lagoon Morphodynamics

6. Salvatore Cesario, Chiara Fredianelli, Alessandro Remorini, Un pacchetto evidence based di tecniche cognitivocomportamentali sui generis

7. Marco Masseti, Uomini e (non solo) topi. Gli animali domestici e la fauna antropocora

8. Simone Margherini (a cura di), BIL Bibliografia Informatizzata Leopardiana 1815-1999: manuale d'uso ver. 1.0

9. Paolo Puma, Disegno dell'architettura. Appunti per la didattica

10. Antonio Calvani (a cura di), Innovazione tecnologica e cambiamento dell'università. Verso l'università virtuale

11. Leonardo Casini, Enrico Marone, Silvio Menghini, La riforma della Politica Agricola Comunitaria e la filiera olivicolo-olearia italiana

12. Salvatore Cesario, L'ultima a dover morire è la speranza. Tentativi di narrativa autobiografica e di "autobiografia assistita"

13. Alessandro Bertirotti, L'uomo, il suono e la musica

14. Maria Antonietta Rovida, Palazzi senesi tra '600 e '700. Modelli abitativi e architettura tra tradizione e innovazione
15. Simone Guercini, Roberto Piovan, Schemi di negoziato e tecniche di comunicazione per il tessile e abbigliamento

16. Antonio Calvani, Technological innovation and change in the university. Moving towards the Virtual University

17. Paolo Emilio Pecorella, Tell Barri/Kahat: la campagna del 2000. Relazione preliminare

18. Marta Chevanne, Appunti di Patologia Generale. Corso di laurea in Tecniche di Radiologia Medica per Immagini e Radioterapia

19. Paolo Ventura, Città e stazione ferroviaria

20. Nicola Spinosi, Critica sociale e individuazione

21. Roberto Ventura (a cura di), Dalla misurazione dei servizi alla customer satisfaction

22. Dimitra Babalis (a cura di), Ecological Design for an Effective Urban Regeneration

23. Massimo Papini, Debora Tringali (a cura di), Il pupazzo di garza. L'esperienza della malattia potenzialmente mortale nei bambini e negli adolescenti

24. Manlio Marchetta, La progettazione della città portuale. Sperimentazioni didattiche per una nuova Livorno

25. Fabrizio F.V. Arrigoni, Note su progetto e metropoli

26. Leonardo Casini, Enrico Marone, Silvio Menghini, OCM seminativi: tendenze evolutive e assetto territoriale

27. Pecorella Paolo Emilio, Raffaella Pierobon Benoit, Tell Barri/Kahat: la campagna del 2001. Relazione preliminare

28. Nicola Spinosi, Wir Kinder. La questione del potere delle relazione adulti/bambini

29. Stefano Cordero di Montezemolo, I profili finanziari delle società vinicole

30. Luca Bagnoli, Maurizio Catalano, Il bilancio sociale degli enti non profit: esperienze toscane 
31. Elena Rotelli, Il capitolo della cattedrale di Firenze dalle origini al XV secolo

32. Leonardo Trisciuzzi, Barbara Sandrucci, Tamara Zappaterra, Il recupero del sé attraverso l'autobiografia

33. Nicola Spinosi, Invito alla psicologia sociale

34. Raffaele Moschillo, Laboratorio di disegno. Esercitazioni guidate al disegno di arredo

35. Niccolò Bellanca, Le emergenze umanitarie complesse. Un'introduzione

36. Giovanni Allegretti, Porto Alegre una biografia territoriale. Ricercando la qualità urbana a partire dal patrimonio sociale

37. Riccardo Passeri, Leonardo Quagliotti, Christian Simoni, Procedure concorsuali e governo dell'impresa artigiana in Toscana

38. Nicola Spinosi, Un soffitto viola. Psicoterapia, formazione, autobiografia

39. Tommaso Urso, Una biblioteca in divenire. La biblioteca della Facoltà di Lettere dalla penna all'elaboratore. Seconda edizione rivista e accresciuta

40. Paolo Emilio Pecorella, Raffaella Pierobon Benoit, Tell Barri/Kahat: la campagna del 2002. Relazione preliminare

41. Antonio Pellicanò, Da Galileo Galilei a Cosimo Noferi : verso una nuova scienza. Un inedito trattato galileiano di architettura nella Firenze del 1650

42. Aldo Burresi (a cura di), Il marketing della moda. Temi emergenti nel tessile-abbigliamento

43. Curzio Cipriani, Appunti di museologia naturalistica

44. Fabrizio F.V. Arrigoni, Incipit. Esercizi di composizione architettonica

45. Roberta Gentile, Stefano Mancuso, Silvia Martelli, Simona Rizzitelli, Il Giardino di Villa Corsini a Mezzomonte. Descrizione dello stato di fatto e proposta di restauro conservativo

46. Arnaldo Nesti, Alba Scarpellini (a cura di), Mondo democristiano, mondo cattolico nel secondo Novecento italiano

47. Stefano Alessandri, Sintesi e discussioni su temi di chimica generale

48. Gianni Galeota (a cura di), Traslocare, riaggregare, rifondare. Il caso della Bib- lioteca di Scienze Sociali dell'Università di Firenze

49. Gianni Cavallina, Nuove città antichi segni. Tre esperienze didattiche

50. Bruno Zanoni, Tecnologia alimentare 1. La classe delle operazioni unitarie di disidratazione per la conservazione dei prodotti alimentari

51. Gianfranco Martiello, La tutela penale del capitale sociale nelle società per azioni

52. Salvatore Cingari (a cura di), Cultura democratica e istituzioni rappresentative. Due esempi a confronto: Italia e Romania

53. Laura Leonardi (a cura di), Il distretto delle donne

54. Cristina Delogu (a cura di), Tecnologia per il web learning. Realtà e scenari

55. Luca Bagnoli (a cura di), La lettura dei bilanci delle Organizzazioni di Volontariato toscane nel biennio 2004-2005

56. Lorenzo Grifone Baglioni (a cura di), Una generazione che cambia. Civismo, solidarietà e nuove incertezze dei giovani della provincia di Firenze

57. Monica Bolognesi, Laura Donati, Gabriella Granatiero, Acque e territorio. Progetti e regole per la qualità dell'abitare

58. Carlo Natali, Daniela Poli (a cura di), Città e territori da vivere oggi e domani. Il contributo scientifico delle tesi di laurea

59. Riccardo Passeri, Valutazioni imprenditoriali per la successione nell'impresa familiare

60. Brunetto Chiarelli, Alberto Simonetta, Storia dei musei naturalistici fiorentini

61. Gianfranco Bettin Lattes, Marco Bontempi (a cura di), Generazione Erasmus? L'identità europea tra vissuto e istituzioni

62. Paolo Emilio Pecorella, Raffaella Pierobon Benoit, Tell Barri / Kahat. La campagna del 2003

63. Fabrizio F.V. Arrigoni, Il cervello delle passioni. Dieci tesi di Adolfo Natalini

64. Saverio Pisaniello, Esistenza minima. Stanze, spazî della mente, reliquiario

65. Maria Antonietta Rovida (a cura di), 
Fonti per la storia dell'architettura, della città, del territorio

66. Ornella De Zordo, Saggi di anglistica e americanistica. Temi e prospettive di ricerca

67. Chiara Favilli, Maria Paola Monaco, Materiali per lo studio del diritto antidiscriminatorio

68. Paolo Emilio Pecorella, Raffaella Pierobon Benoit, Tell Barri / Kahat. La campagna del 2004

69. Emanuela Caldognetto Magno, Federica Cavicchio, Aspetti emotivi e relazionali nell'e-learning

70. Marco Masseti, Uominie (non solo) topi ( $2^{\mathrm{a}}$ edizione)
71. Giovanni Nerli, Marco Pierini, Costruzione di macchine

72. Lorenzo Viviani, L'Europa dei partiti. Per una sociologia dei partiti politici nel processo di integrazione europea

73. Teresa Crespellani, Terremoto e ricerca. Un percorso scientifico condiviso per la caratterizzazione del comportamento sismico di alcuni depositi italiani

74. Fabrizio F.V. Arrigoni, Cava. Architettura in "ars marmoris"

75. Ernesto Tavoletti, Higher Education and Local Economic Development

76. Carmelo Calabrò, Liberalismo, democrazia, socialismo. L'itinerario di Carlo Rosselli (1917-1930) 
